\title{
Asymmetric Copper-Catalyzed Carbozincation of Cyclopropenes \\ en route to the Formation of \\ Diastereo- and Enantiomerically Enriched Polysubstituted Cyclopropanes
}

\begin{abstract}
Authors: Daniel S. Müller and Ilan Marek
The Mallat Family Laboratory of Organic Chemistry, Schulich Faculty of Chemistry, and The Lise Meitner-Minerva Center for Computational Quantum Chemistry, Technion-Israel Institute of Technology, Technion City, Haifa 3200o, Israel
\end{abstract}

Table of contents

$1 \quad$ General experimental details $\quad$ S2

2 Optimization reactions $\quad$ S3

3 Substrate Synthesis $\quad$ S5

$4 \quad \mathrm{Cu}$-catalyzed enantioselective carbozincation of cyclopropenes $\quad \mathrm{S} 6$

$\begin{array}{lll}5 & \text { References } & \text { S24 }\end{array}$

$\begin{array}{lll}6 & \text { NMR spectra } & \text { S25 }\end{array}$ 
Unless stated otherwise, reactions were conducted in oven-dried glassware under an atmosphere of argon. TMSCl was purified by distillation under a nitrogen atmosphere over $\mathrm{CaH}_{2}$. Methyl vinyl ketone was purified by vacuum distillation. Ether and THF were dried from Pure-Solv ${ }^{\circledR}$ Purification System (Innovative Technology ${ }^{\circledR}$ ). All other commercially obtained reagents were used as received. Thin-layer chromatography (TLC) was conducted with E. Merck silica gel 60 F254 pre-coated plates, $(0.25 \mathrm{~mm})$ and visualized by exposure to UV light $(254 \mathrm{~nm})$ or stained with anisaldehyde, phosphomolybdic acid, or potassium permanganate. Column chromatography was performed using Fluka silica gel $60 \AA$ (40-63 $\mu \mathrm{m}, 230-400$ mesh). ${ }^{1} \mathrm{H}$ NMR spectra were recorded on Bruker spectrometers (AVIII400) and are reported relative to deuterated solvent signals. Chemical shifts are reported in parts per million (ppm) with respect to the residual solvent signal $\mathrm{CDCl}_{3}\left({ }^{1} \mathrm{H}\right.$ NMR: $\delta=7.26$; 13C NMR: $\delta=77.16$ ). Peak multiplicities are reported as follows: s $=$ singlet, $\mathrm{bs}=$ broad singlet, $\mathrm{d}=$ doublet, $\mathrm{t}=$ triplet, $\mathrm{dd}=$ doublet of doublets, $\mathrm{td}=$ triplet of doublets, $\mathrm{m}$ $=$ multiplet. app =apparent. High-resolution mass spectra (HRMS) were obtained by the mass spectrometry facility at the Technion. Reactions were monitored by gas chromatography spectrometry (GC) using an Agilent Technologies 7820A GC with an Agilent Technologies 19091J-413 (30 m $\times 0.3 \mathrm{~mm})$ column or (GC-MS) Thermo Scientific ${ }^{\text {TM }}$ Ion Trap GC/MS: $\mathrm{ITQ}^{\mathrm{TM}} 900$ with a Varian Factor Four Capilary colum $(\mathrm{VF}-5 \mathrm{~ms}, 30 \mathrm{~m} \times 0.25 \mathrm{~mm})$. HPLC chromatograms were recorded using Agilent $(\mathrm{C} 1100$ Series line. Following chiral columns were tested: CHIRAL PAK ${ }^{\circledR}$ AD-H $(0.46 \mathrm{~cm} \varnothing \times 25 \mathrm{~cm})$, CHIRALCEL $^{\circledR}$ OD $(0.46 \mathrm{~cm} \varnothing \times 25$ $\mathrm{cm})$ and CHIRALCEL $\AA$ IA column $(0.46 \mathrm{~cm} \varnothing \times 25 \mathrm{~cm})$. Enantiomeric excesses were determined by chiral-GC: Varian 3900 with J\&W Scientific Cycldodex B $(30 \mathrm{~m} \times 0.32 \mathrm{~mm})$ or FS-Lipodex E $(25 \mathrm{~m} \times 0.25 \mathrm{~mm})$. Temperature programs for chiral GC are described as follows: initial temperature $\left({ }^{\circ} \mathrm{C}\right)$-initial time $(\mathrm{min})$-temperature gradient $\left({ }^{\circ} \mathrm{C} / \mathrm{min}\right)$-final temperature $\left({ }^{\circ} \mathrm{C}\right)$. Optical rotations were measured on a SCHMIDT and HAENSCH ${ }^{\odot}$ Unipol L1000 polarimeter with $[\alpha] \mathrm{D}$ values reported in degrees; concentration (c) is in $\mathrm{g} / 100 \mathrm{~mL}$. Racemic samples were obtained by carrying out the reaction under conditions indicated for the optically enriched cyclopropane $3 \mathbf{a}$ using a 1:1 mixture of $(R)$ - and $(S)$-DTBM-SEGPHOS (5.5 mol\%, each) and $\mathrm{Cu}\left(\mathrm{CH}_{3} \mathrm{CN}\right)_{4} \mathrm{PF}_{6}(10 \mathrm{~mol} \%)$. 
Table S1: Initial screening.<smiles>c1ccc(P(c2ccccc2)c2ccccc2)cc1</smiles>

TPP<smiles>Pc1cccc(Oc2ccccc2-c2ccccc2)c1-c1ccccc1</smiles>

DPEPhos<smiles>c1ccc(-c2ccc3ccccc3c2-c2c(P(c3ccccc3)c3ccccc3)ccc3ccccc23)cc1</smiles>

(R)-BINAP

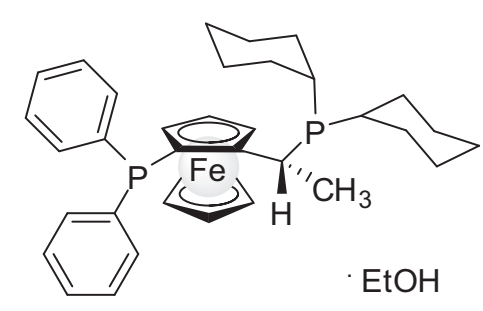

Josiphos<smiles>O=C(NC1CCCCC1)c1ccccc1-c1ccccc1</smiles>

$(R, R)$-Dach Ligand; Trost Ligand<smiles>CC(c1ccccc1)N([C@@H](C)c1ccccc1)P(Oc1ccccc1)Oc1ccc2ccccc2c1-c1c(O)ccc2ccccc12</smiles>

$S, R, R$ - Feringa Ligand

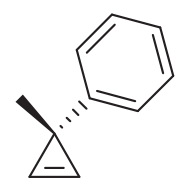

1a
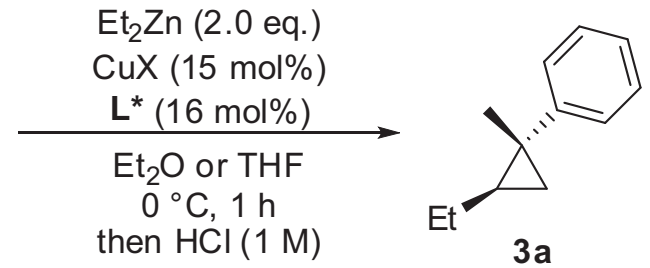

\begin{tabular}{|c|c|c|c|c|c|c|}
\hline Entry $^{[\mathrm{a}]}$ & Solvent & $\mathrm{CuX}$ & Ligand & Conv. ${ }^{[\mathrm{b}]}$ & d.r. ${ }^{[b]}$ & $\mathrm{er}^{[\mathrm{c}]}$ \\
\hline 1 & $\mathrm{Et}_{2} \mathrm{O}$ & $\mathrm{CuI}$ & TPP & $<20 \%$ & n.d. & -- \\
\hline 2 & $\mathrm{Et}_{2} \mathrm{O}$ & $\mathrm{CuI}$ & DPEPHOS & $<20 \%$ & n.d. & -- \\
\hline 3 & $\mathrm{Et}_{2} \mathrm{O}$ & $\mathrm{CuI}$ & $(R)$-BINAP & $<20 \%$ & n.d. & -- \\
\hline 4 & THF & $\mathrm{CuI}$ & -- & $100 \%$ & $4: 1$ & -- \\
\hline 5 & $\mathrm{Et}_{2} \mathrm{O}$ & $\mathrm{Cu}\left(\mathrm{CH}_{3} \mathrm{CN}\right)_{4} \mathrm{PF}_{6}$ & ТPP & $100 \%$ & $1.7: 1$ & -- \\
\hline 6 & $\mathrm{Et}_{2} \mathrm{O}$ & $\mathrm{Cu}\left(\mathrm{CH}_{3} \mathrm{CN}\right)_{4} \mathrm{PF}_{6}$ & DPEPHOS & $<20 \%$ & n.d. & -- \\
\hline 7 & $\mathrm{Et}_{2} \mathrm{O}$ & $\mathrm{Cu}\left(\mathrm{CH}_{3} \mathrm{CN}\right)_{4} \mathrm{PF}_{6}$ & $(R)$-BINAP & $68 \%$ & $1: 1.7$ & n.d. \\
\hline 8 & $\mathrm{Et}_{2} \mathrm{O}$ & $\mathrm{CuTC}$ & ТPP & $100 \%$ & $1.7: 1$ & -- \\
\hline 9 & $\mathrm{Et}_{2} \mathrm{O}$ & $\mathrm{CuTC}$ & DPEPHOS & $<20 \%$ & n.d. & -- \\
\hline 10 & $\mathbf{E t}_{2} \mathbf{O}$ & CuTC & $(R)$-BINAP & $100 \%$ & $15: 1$ & $84: 16$ \\
\hline 11 & THF & $\mathrm{CuTC}$ & $(R)$-BINAP & $100 \%$ & $20: 1$ & $81: 19$ \\
\hline 12 & THF & $\mathrm{CuTC}$ & -- & $100 \%$ & $4: 1$ & -- \\
\hline 13 & $\mathrm{Et}_{2} \mathrm{O}$ & CuTC & -- & $100 \%$ & $3: 1$ & -- \\
\hline 14 & $\mathrm{Et}_{2} \mathrm{O}$ & $\mathrm{CuTC}$ & Josiphos & $<20 \%$ & n.d. & n.d. \\
\hline 15 & $\mathrm{Et}_{2} \mathrm{O}$ & $\mathrm{CuTC}$ & Trost-Ligand & $<20 \%$ & n.d. & n.d. \\
\hline 16 & $\mathrm{Et}_{2} \mathrm{O}$ & $\mathrm{CuTC}$ & Feringa-Ligand & $100 \%$ & $1.6: 1.0$ & $74: 26$ \\
\hline 17 & THF & $\mathrm{CuTC}$ & Feringa-Ligand & $100 \%$ & $5: 1$ & $58: 42$ \\
\hline
\end{tabular}

[a] Ligand, copper salt and solvent were stirred for $1 \mathrm{~h}$ at $\mathrm{rt}$ then cyclopropene was added followed by $\mathrm{Et}_{2} \mathrm{Zn}$. In all cases $10-50 \%$ dimer side-product was observed. [b] Estimated by GC-MS. [d] Determined by chiral GC with Cyclodex-B as stationary phase. 
Table S2: $\quad$ Screening of copper salt

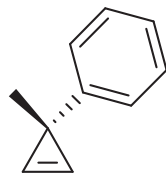

1a

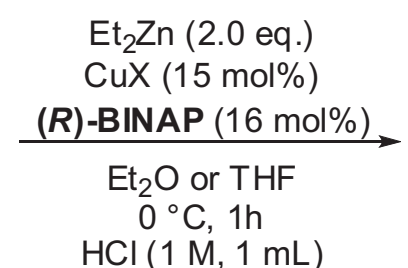

CuX $(15 \mathrm{~mol} \%)$

$\boldsymbol{R})$-BINAP (16 mol\%)

$\mathrm{Et}_{2} \mathrm{O}$ or THF

$\mathrm{HCl}(1 \mathrm{M}, 1 \mathrm{~mL})$

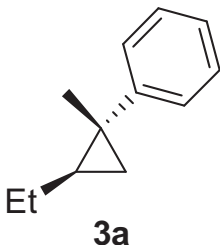

$3 a$

\begin{tabular}{cccccc}
\hline Entry $^{[\mathrm{a}]}$ & Solvent & $\mathrm{CuX}$ & Conv. $^{[\mathrm{b}]}$ & d.r. $^{[\mathrm{b}]}$ & er $^{[\mathrm{c}]}$ \\
\hline 1 & $\mathrm{Et}_{2} \mathrm{O}$ & $\mathrm{CuI}$ & $<20 \%$ & n.d. & -- \\
2 & $\mathrm{Et} 2 \mathrm{O}$ & $\mathrm{Cu}\left(\mathrm{CH}_{3} \mathrm{CN}\right)_{4} \mathrm{PF}_{6}$ & $68 \%$ & $1: 1.7$ & n.d. \\
3 & $\mathrm{THF}$ & $\mathrm{Cu}\left(\mathrm{CH}_{3} \mathrm{CN}\right)_{4} \mathrm{PF}_{6}$ & $100 \%$ & $19: 1$ & $77: 23$ \\
$\mathbf{4}$ & $\mathbf{E t}_{2} \mathbf{O}$ & $\mathbf{C u T C}$ & $\mathbf{1 0 0 \%}$ & $\mathbf{1 5 : 1}$ & $\mathbf{8 4 : 1 6}$ \\
5 & $\mathrm{THF}$ & $\mathrm{CuTC}$ & $100 \%$ & $20: 1$ & $81: 19$ \\
6 & $\mathrm{Et} t_{2} \mathrm{O}$ & $\mathrm{CuBr}$ & $<20 \%$ & n.d. & n.d. \\
7 & $\mathrm{THF}$ & $\mathrm{CuBrSMe} \mathrm{SM}_{2}$ & $<20 \%$ & n.d. & n.d. \\
8 & $\mathrm{Et}_{2} \mathrm{O}$ & $\mathrm{CuBr} \cdot \mathrm{SMe}_{2}$ & $<20 \%$ & n.d. & n.d. \\
9 & $\mathrm{Et}_{2} \mathrm{O}$ & $\mathrm{Cu}(\mathrm{OTf})_{2}$ & $11 \%$ & n.d. & $55: 45$ \\
10 & $\mathrm{Et}_{2} \mathrm{O}$ & $\mathrm{Cu}(\mathrm{OAc})_{2} \mathrm{H}_{2} \mathrm{O}$ & $100 \%$ & $16: 1$ & $81: 19$ \\
11 & $\mathrm{Et}_{2} \mathrm{O}$ & $\mathrm{CuCl}$ & $<20 \%$ & n.d. & n.d. \\
\hline
\end{tabular}

[a] Ligand, copper salt and solvent were stirred for $1 \mathrm{~h}$ at $\mathrm{rt}$ then cyclopropene was added followed by $\mathrm{Et}_{2} \mathrm{Zn}$. In all cases $10-30 \%$ dimer side-product was observed. [b] Estimated by GC-MS. [d] Determined by chiral GC with Cyclodex-B as stationary phase.

Table S3: $\quad$ Screening of solvents

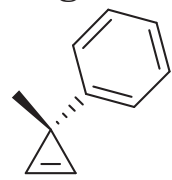

$1 \mathrm{a}$

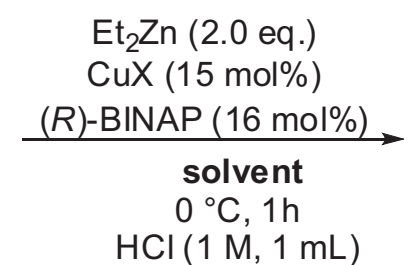

$\mathrm{Et}_{2} \mathrm{Zn}(2.0 \mathrm{eq}$.

Cl $(1 \mathrm{M}, 1 \mathrm{~mL})$

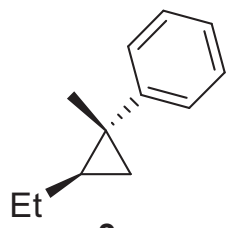

$3 a$

\begin{tabular}{ccccc}
\hline Entry $^{[\mathrm{a}]}$ & Solvent & Conv. & d.r. $^{[\mathrm{b}]}$ & er $^{[\mathrm{c}]}$ \\
\hline $\mathbf{1}$ & $\mathbf{E t}_{\mathbf{2}} \mathbf{O}$ & $\mathbf{1 0 0 \%}$ & $\mathbf{1 5 : 1}$ & $\mathbf{8 4 : 1 6}$ \\
2 & $\mathrm{THF}$ & $100 \%$ & $20: 1$ & $81: 19$ \\
$\mathbf{3}$ & $\mathbf{E t O A c}$ & $\mathbf{1 0 0 \%}$ & $\mathbf{2 3 : 1}$ & $\mathbf{8 5 : 1 5}$ \\
4 & $\mathrm{CH}_{2} \mathrm{Cl}_{2}$ & $100 \%$ & $22: 1$ & $74: 26$ \\
5 & $\mathrm{MTBE}$ & $100 \%$ & $19: 1$ & $80: 20$ \\
6 & toluene & $100 \%$ & $20: 1$ & $83: 17$ \\
\hline
\end{tabular}

[a] Ligand, copper salt and solvent were stirred for $1 \mathrm{~h}$ at $\mathrm{rt}$ then cyclopropene was added followed by $\mathrm{Et}_{2} \mathrm{Zn}$. In all cases $10-30 \%$ dimer side-product was observed. [b] Estimated by GC-MS. [c] Determined by chiral GC with Cyclodex-B as stationary phase.

Note: Even though ethyl acetate gave slightly better results compared to diethyl ether we continued our investigations with diethyl ether. Dry diethyl ether was available from the solvent system which was not the case for ethyl acetate. 
The substrates were prepared as shown in table S4. The procedures described in the references were followed.

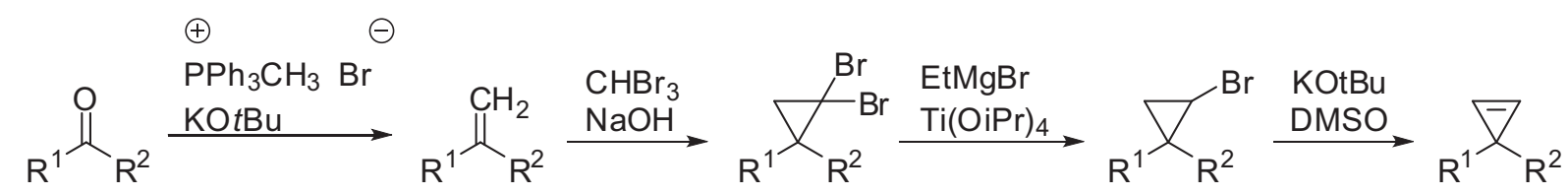

In some cases the olefin was commercially available.

Table S4: $\quad$ Substrate synthesis

\begin{tabular}{|c|c|c|}
\hline Substrate & Structure & Reference for synthesis \\
\hline $1 \mathrm{a}$ & & $\begin{array}{l}\text { D. H. T. Phan, K. G. M. Kou, V. M. Dong, } \\
\text { J Am Chem Soc 2010, 132, 16354-16355 }\end{array}$ \\
\hline $1 \mathrm{~b}$ & & $\begin{array}{l}\text { W. M. Sherrill, R. Kim, M. Rubin, } \\
\text { Tetrahedron 2008, 64, 8610-8617. }\end{array}$ \\
\hline cc & & $\begin{array}{l}\text { W. M. Sherrill, R. Kim, M. Rubin, } \\
\text { Tetrahedron 2008, 64, 8610-8617. }\end{array}$ \\
\hline 1d & & $\begin{array}{l}\text { A. Parra, L. Amenós, M. Guisán-Ceinos, A. López, } \\
\text { J. L. Ruano, M. Tortosa, } \\
\text { J. Am. Chem. Soc. 2014, 136, 15833-15836. }\end{array}$ \\
\hline $1 \mathrm{e}$ & & $\begin{array}{l}\text { D. H. T. Phan, K. G. M. Kou, V. M. Dong, } \\
\text { J Am Chem Soc 2010, 132, 16354-16355 }\end{array}$ \\
\hline 1f & & $\begin{array}{l}\text { A. Parra, L. Amenós, M. Guisán-Ceinos, A. López, } \\
\text { J. L. Ruano, M. Tortosa, } \\
\text { J. Am. Chem. Soc. 2014, 136, 15833-15836. }\end{array}$ \\
\hline $1 g$ & & $\begin{array}{l}\text { K. Kramer, P. Leong, M. Lautens, } \\
\text { Org Lett 2011, 13, 819-821. }\end{array}$ \\
\hline 1h & & $\begin{array}{l}\text { R. E. Giudici, A. H. Hoveyda, } \\
\text { J. Am. Chem. Soc. 2007, 129, 3824-3825. }\end{array}$ \\
\hline
\end{tabular}




\section{((1R,2R)-2-ethyl-1-methylcyclopropyl)benzene (3a)}

\section{General procedure GP1.}

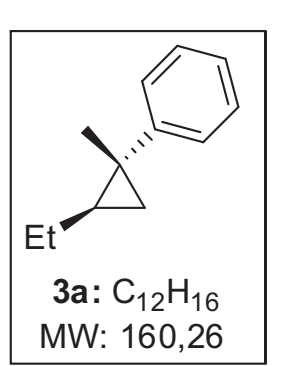

$\mathrm{Cu}\left(\mathrm{CH}_{3} \mathrm{CN}\right)_{4} \mathrm{PF}_{6} \quad(11.2 \mathrm{mg}, 0.03 \mathrm{mmol}, 3 \mathrm{~mol} \%)$ and $(R)$-DTBMSEGPHOS $^{\circledR}$ (38.9 mg, $0.033 \mathrm{mmol}, 3.3 \mathrm{~mol} \%$ ) were dissolved in THF (4.0 $\mathrm{mL}$ ) under argon atmosphere. After stirring the pale pink solution for $1 \mathrm{~h}$ at rt the mixture was cooled to $0{ }^{\circ} \mathrm{C}$ and the cyclopropene substrate 1a $(2.0$ $\mathrm{mL}, 130.2 \mathrm{mg}, 1.0 \mathrm{mmol}, 1.0$ eq.; $0.5 \mathrm{M}$ solution in pentane) was added. After 5 min $\mathrm{Et}_{2} \mathrm{Zn}(1.2 \mathrm{~mL}, 1.2 \mathrm{mmol}, 1.2$ eq., $1.0 \mathrm{M}$ solution in hexanes) was added dropwise at the wall of the flask. After $3 \mathrm{~h}$ at $0{ }^{\circ} \mathrm{C}$ the reaction mixture was quenched with $\mathrm{HCl}(1.0 \mathrm{M} ; 5.0 \mathrm{~mL})$ and diluted with $\mathrm{Et}_{2} \mathrm{O}(10 \mathrm{~mL})$. The organic phase was separated and the aqueous phase extracted with $\mathrm{Et}_{2} \mathrm{O}(10 \mathrm{~mL})$. The combined organic phases were dried over $\mathrm{Na}_{2} \mathrm{SO}_{4}$ and reduced in vacuo. The oily residue was purified by flash chromatography (hexanes) to obtain $\mathbf{3 a}$ as a colorless oil (136 mg, $0.85 \mathrm{mmol}, 85 \%$ yield). $\mathrm{R}_{\mathrm{f}}$ $=0.79$ (Hexanes; stained with phosphomolybdic acid).

${ }^{1} \mathbf{H}$ NMR $\left(400 \mathrm{MHz}, \mathrm{CDCl}_{3}\right) \delta 7.27-7.19(\mathrm{~m}, 4 \mathrm{H}), 7.14-7.08(\mathrm{~m}, 1 \mathrm{H}), 1.54-1.41(\mathrm{~m}, 2 \mathrm{H})$, 1.35 (s, 3H), 1.04 (t, $J=7.3 \mathrm{~Hz}, 3 \mathrm{H}), 1.01$ (d, $J=4.3 \mathrm{~Hz}, 1 \mathrm{H}), 0.99-0.89$ (m, 1H), 0.34 (dd, $J=5.4,4.6 \mathrm{~Hz}, 1 \mathrm{H}) .{ }^{13} \mathbf{C}$ NMR $\left(101 \mathrm{MHz}, \mathrm{CDCl}_{3}\right) \delta 149.1,128.3,127.1,125.4,28.4,24.3$, 23.0, 20.7, 20.3, 14.6. Spectral data consistent with reported data. ${ }^{[1]} \mathrm{GC}$ analysis (Cyclodex-B; 60-1-70-0.4-82-180; $\left.\mathrm{t}_{\mathrm{R} 1}=34.1 \mathrm{~min}, \mathrm{t}_{\mathrm{R} 2}=34.9 \mathrm{~min}\right)$ indicated $93: 7$ er. $[\alpha]_{\mathrm{D}}{ }^{20}=-23.1(\mathrm{c}=$ $\left.0.48, \mathrm{CHCl}_{3}\right)$. 


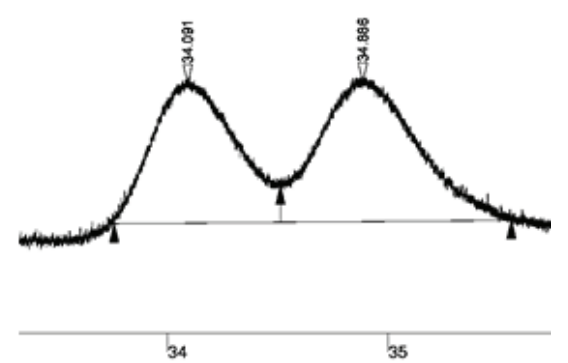

\begin{tabular}{ccc}
$\begin{array}{c}\text { Ret. } \\
\text { Time } \\
\text { (min) }\end{array}$ & $\begin{array}{c}\text { Time } \\
\text { Offset } \\
\text { (min) }\end{array}$ & $\begin{array}{c}\text { Area } \\
\text { (counts) }\end{array}$ \\
\cline { 1 - 1 } & 0.000 & 6399 \\
34.886 & 0.000 & 6924 \\
\hline
\end{tabular}

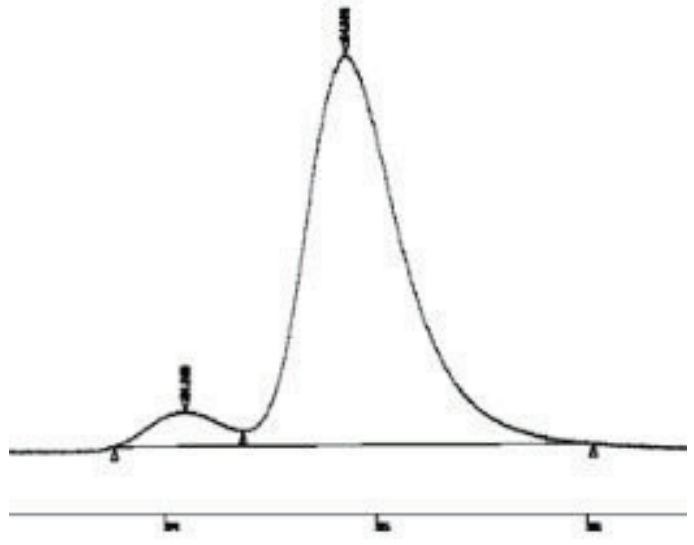

\begin{tabular}{|c|c|c|}
\hline $\begin{array}{l}\text { Ret. } \\
\text { Time } \\
\text { (min) }\end{array}$ & $\begin{array}{l}\text { Time } \\
\text { Offset } \\
\text { (min) }\end{array}$ & $\begin{array}{c}\text { Area } \\
\text { (counts) }\end{array}$ \\
\hline 34.098 & 0.000 & 5663 \\
\hline 34.856 & 0.000 & 89286 \\
\hline
\end{tabular}

\section{$((1 R, 2 S, 3 S)$-2-ethyl-3-iodo-1-methylcyclopropyl)benzene (3b)}

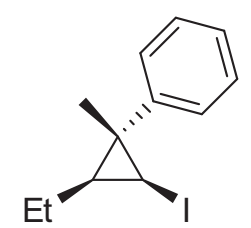

3b: $\mathrm{C}_{12} \mathrm{H}_{15} \mathrm{l}$ MW: 286,15

To cyclopropyl zinc reagent $(1.0 \mathrm{mmol}$; prepared according to general procedure GP1) was added iodine (914 mg, $3.6 \mathrm{mmol}, 3.6$ eq.) at $0{ }^{\circ} \mathrm{C}$. The reaction was stirred for $4 \mathrm{~h}$ at this temperature and then quenched with sat. aqueous solution of $\mathrm{Na}_{2} \mathrm{~S}_{2} \mathrm{O}_{3}(10 \mathrm{~mL})$ and diluted with $\mathrm{Et}_{2} \mathrm{O}$ $(10 \mathrm{~mL})$. The organic phase was separated and the aqueous phase extracted with $\mathrm{Et}_{2} \mathrm{O}(10 \mathrm{~mL})$. The combined organic phases were dried over $\mathrm{Na}_{2} \mathrm{SO}_{4}$ and reduced in vacuo. The oily residue was purified by flash chromatography (hexanes) to obtain $\mathbf{3 b}$ as a pale pink oil (277 $\mathrm{mg}, 0.97 \mathrm{mmol}, 97 \%$ yield). $\mathrm{R}_{\mathrm{f}}=0.64$ (Hexanes; stained with phosphomolybdic acid).

${ }^{1}$ H NMR $\left(400 \mathrm{MHz}, \mathrm{CDCl}_{3}\right) \delta 7.22-7.14(\mathrm{~m}, 4 \mathrm{H}), 7.13-7.07(\mathrm{~m}, 1 \mathrm{H}), 3.07(\mathrm{~d}, J=8.2 \mathrm{~Hz}$, $1 \mathrm{H}), 1.50-1.32(\mathrm{~m}, 2 \mathrm{H}), 1.30(\mathrm{~s}, 3 \mathrm{H}), 1.01(\mathrm{t}, J=7.4 \mathrm{~Hz}, 3 \mathrm{H}), 0.99-0.93(\mathrm{~m}, 1 \mathrm{H})$. ${ }^{13}$ C NMR $\left(101 \mathrm{MHz}, \mathrm{CDCl}_{3}\right) \delta 146.9,128.6,127.3,126.4,28.7,27.4,23.6,22.3,13.1,12.3$.

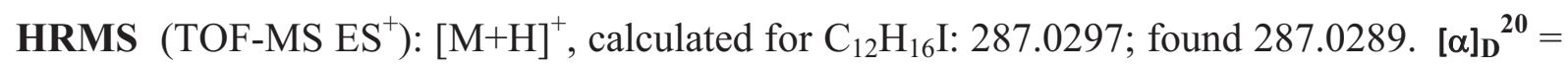
$+52.1\left(\mathrm{c}=0.51, \mathrm{CHCl}_{3}\right)$.

Note: The enantiomeric ratio of $\mathbf{3 b}$ could not be determined by chiral HPLC or GC (see chemical materials and methods). Therefore, the same enantiomeric ratio as for $\mathbf{3 a}$ was assigned. For proof that transmetalation does not change the enantiomeric ratio see compounds $\mathbf{3 j}, \mathbf{3 k}, \mathbf{3 s}$ and $\mathbf{3 t}$. 
((1S,2R,3S)-2-ethyl-1-methyl-3-styrylcyclopropyl)benzene (3c)

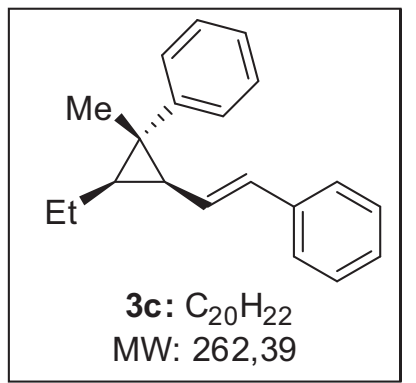

To cyclopropyl zinc reagent $(0.6 \mathrm{mmol}$; prepared according to general procedure GP1) was added $(E)$-(2-bromovinyl)benzene ${ }^{[2]}$ (193 $\mu \mathrm{L}, 274 \mathrm{mg}, 1.5 \mathrm{mmol}, 2.5$ eq.) and a solution of $\mathrm{Pd}(\mathrm{OAc})_{2}$ (4.0 mg, $0.018 \mathrm{mmol}, 0.03$ eq.) and SPhos (14.8 mg, $0.036 \mathrm{mmol}$, 0.06 eq. $)$ in THF $(0.5 \mathrm{~mL})$ at $0{ }^{\circ} \mathrm{C}$. Stirring was continued for $16 \mathrm{~h}$ during which the cooling bath slowly warmed to $20{ }^{\circ} \mathrm{C}$. The reaction mixture was treated with $\mathrm{HCl}(1 \mathrm{M}, 5 \mathrm{~mL})$ and extracted with $\mathrm{Et}_{2} \mathrm{O}(3 \times 15 \mathrm{~mL})$. The combined organic phases were dried over $\mathrm{Na}_{2} \mathrm{SO}_{4}$ and the reduced in vacuo. The oily residue was purified by flash chromatography (hexanes) to obtain 3c as a colorless oil (145 $\mathrm{mg}, 0.55$ mmol, 92\% yield). $\mathrm{R}_{\mathrm{f}}=0.35$ (Hexanes; UV detection).

${ }^{1} \mathbf{H}$ NMR $\left(400 \mathrm{MHz}, \mathrm{CDCl}_{3}\right) \delta 7.41-7.35(\mathrm{~m}, 2 \mathrm{H}), 7.35-7.28(\mathrm{~m}, 6 \mathrm{H}), 7.24-7.16(\mathrm{~m}, 2 \mathrm{H})$, $6.63(\mathrm{~d}, J=15.7 \mathrm{~Hz}, 1 \mathrm{H}), 6.17(\mathrm{dd}, J=15.7,9.7 \mathrm{~Hz}, 1 \mathrm{H}), 1.98(\mathrm{t}, J=9.4 \mathrm{~Hz}, 1 \mathrm{H}), 1.69-1.61$ $(\mathrm{m}, 2 \mathrm{H}), 1.45(\mathrm{dd}, J=7.6,9.5 \mathrm{~Hz}, 1 \mathrm{H}), 1.42(\mathrm{~s}, 3 \mathrm{H}), 1.11(\mathrm{t}, J=7.3 \mathrm{~Hz}, 3 \mathrm{H}) .{ }^{13} \mathbf{C}$ NMR $(101$ $\left.\mathrm{MHz}_{\mathrm{CDCl}}\right) \delta 149.7,138.1,131.6,128.7,128.5,127.5,127.2,126.8,125.9,125.8,32.8$, $31.8,30.9,18.5,16.4,14.4$. HRMS (APCI): $[\mathrm{M}+\mathrm{H}]^{+}$, calculated for $\mathrm{C}_{20} \mathrm{H}_{23}$ : 263.1794; found 263.1783. $[\alpha]_{\mathbf{D}}{ }^{20}=+252.9\left(\mathrm{c}=1.00, \mathrm{CHCl}_{3}\right)$.

Note: The enantiomeric ratio of $3 \mathbf{c}$ could not be determined by HPLC or GC with the chiral columns tested (see chemical materials and methods). Therefore, the same enantiomeric ratio as for $\mathbf{3 a}$ was assigned.

\section{((1S,2S,3R)-2-allyl-3-ethyl-1-methylcyclopropyl)benzene (3d)}

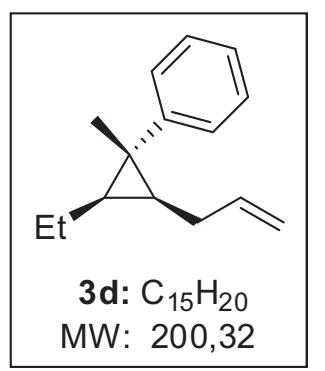

To cyclopropyl zinc reagent $(1.0 \mathrm{mmol}$; prepared according to general procedure GP1) was added $\mathrm{CuCN} 2 \mathrm{LiCl}(0.5 \mathrm{M}$ in THF; $0.96 \mathrm{~mL}, 0.48$ mmol, 0.48 eq.) at $-40{ }^{\circ} \mathrm{C}$. After $5 \mathrm{~min}$ allyl bromide $(243 \mu \mathrm{L}, 338 \mathrm{mg}$, 2.8 mmol, 2.8 eq.) was added. After stirring for $1 \mathrm{~h}$ at $-40{ }^{\circ} \mathrm{C}$ the reaction was slowly warmed to $\mathrm{rt}$ and continued to stir for $4 \mathrm{~h}$. The reaction mixture was treated with $\mathrm{HCl}(1 \mathrm{M}, 5 \mathrm{~mL})$ and extracted with $\mathrm{Et}_{2} \mathrm{O}(3 \times 10 \mathrm{~mL})$. The combined organic phases were dried over $\mathrm{Na}_{2} \mathrm{SO}_{4}$ and reduced in vacuo. The oily residue was purified by flash chromatography (hexanes to hexanes: EtOAc $=$ $30: 1)$ to obtain $\mathbf{3 d}$ as a colorless oil $\left(182 \mathrm{mg}, 0.91 \mathrm{mmol}, 91 \%\right.$ yield). $\mathrm{R}_{\mathrm{f}}=0.82$ (Hexanes; stained with $\mathrm{KMnO}_{4}$ ). 
${ }^{1} \mathbf{H}$ NMR $\left(400 \mathrm{MHz}, \mathrm{CDCl}_{3}\right) \delta 7.27-7.22(\mathrm{~m}, 4 \mathrm{H}), 7.16-7.09(\mathrm{~m}, 1 \mathrm{H}), 6.01-5.89(\mathrm{~m}, 1 \mathrm{H})$, $5.11(\mathrm{dd}, J=17.1,1.7 \mathrm{~Hz}, 1 \mathrm{H}), 5.00(\mathrm{dd}, J=10.2,1.6 \mathrm{~Hz}, 1 \mathrm{H}), 2.25-2.11(\mathrm{~m}, 2 \mathrm{H}), 1.55-$ $1.37(\mathrm{~m}, 2 \mathrm{H}), 1.26(\mathrm{~s}, 3 \mathrm{H}), 1.18-1.10(\mathrm{dt}, J=8.0 \mathrm{~Hz}, J=7.4 \mathrm{~Hz}), 1.27-1.20(\mathrm{~m}, 4 \mathrm{H}) .{ }^{13} \mathrm{C}$ NMR $\left(101 \mathrm{MHz}, \mathrm{CDCl}_{3}\right) \delta 150.5,138.6,128.3,127.5,125.5,114.6,29.1,28.7,26.4,26.3$, 17.7, 15.3, 14.6. HRMS (TOF-MS ES ${ }^{+}$): $[\mathrm{M}+\mathrm{H}]^{+}$, calculated for $\mathrm{C}_{15} \mathrm{H}_{21}$ : 201.1643; found 201.1629. $[\alpha]_{\mathbf{D}}{ }^{20}=+1.0\left(\mathrm{c}=0.55, \mathrm{CHCl}_{3}\right)$.

Note: The enantiomeric ratio of $\mathbf{3 d}$ could not be determined by HPLC or GC with the chiral columns tested (see chemical materials and methods). Therefore, the same enantiomeric ratio as for $\mathbf{3 a}$ was assigned.

Ethyl 2-(((1S,2S,3R)-3-ethyl-2-methyl-2-phenylcyclopropyl)methyl)acrylate (3e)

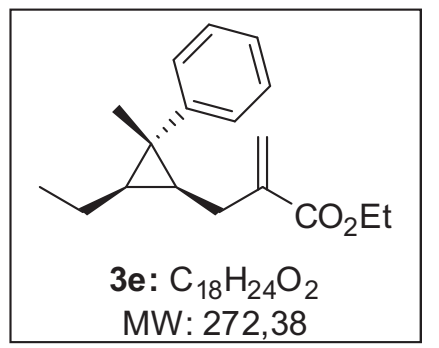

To cyclopropyl zinc reagent $(1.0 \mathrm{mmol}$; prepared according to general procedure GP1) was added $\mathrm{CuCN} 2 \mathrm{LiCl}(0.5 \mathrm{M}$ in THF; $0.96 \mathrm{~mL}, 0.48 \mathrm{mmol}, 0.48$ eq.) at $-40{ }^{\circ} \mathrm{C}$. After $5 \mathrm{~min}$ the ethyl 2(bromomethyl)acrylate (389 $\mu \mathrm{L}, 540 \mathrm{mg}, 2.8 \mathrm{mmol}, 2.8 \mathrm{eq}$.) was added. After stirring for $1 \mathrm{~h}$ at $-40{ }^{\circ} \mathrm{C}$ the reaction was slowly warmed to $\mathrm{rt}$ and continued to stir for $4 \mathrm{~h}$. The reaction was quenched with sat. aqueous solution of $\mathrm{NH}_{4} \mathrm{Cl}(10 \mathrm{~mL})$ and diluted with $\mathrm{Et}_{2} \mathrm{O}(10 \mathrm{~mL})$. The organic phase was separated and the aqueous phase extracted with $\mathrm{Et}_{2} \mathrm{O}(10 \mathrm{~mL})$. The combined organic phases were dried over $\mathrm{Na}_{2} \mathrm{SO}_{4}$ and reduced in vacuo. The oily residue was purified by flash chromatography (hexanes to hexanes: EtOAc $=30: 1$ ) and dried under high vacuum ${ }^{[1]}$ to obtain $3 \mathbf{e}$ as a colorless oil (264 mg, $0.97 \mathrm{mmol}, 97 \%$ yield). $\mathrm{R}_{\mathrm{f}}=0.57$ (Hexanes: EtOAc $=10: 1$; stained with $\mathrm{KMnO}_{4}$ ).

${ }^{1} \mathbf{H}$ NMR $\left(400 \mathrm{MHz}, \mathrm{CDCl}_{3}\right) \delta 7.20-7.13(\mathrm{~m}, 4 \mathrm{H}), 7.07-7.01(\mathrm{~m}, 1 \mathrm{H}), 6.13(\mathrm{~d}, J=1.0 \mathrm{~Hz}$, $1 \mathrm{H}), 5.59$ (d, $J=1.5 \mathrm{~Hz}, 1 \mathrm{H}), 4.13(\mathrm{q}, J=7.1 \mathrm{~Hz}, 2 \mathrm{H}), 2.38$ (dd, $J=7.2,1.0 \mathrm{~Hz}, 2 \mathrm{H}), 1.50-$ $1.26(\mathrm{~m}, 2 \mathrm{H}), 1.21(\mathrm{t}, J=9.0,3 \mathrm{H}), 1.18(\mathrm{~s}, 3 \mathrm{H}), 1.17-1.13(\mathrm{~m}, 1 \mathrm{H}), 1.06-1.00(\mathrm{~m}, 1 \mathrm{H})$, $0.97(\mathrm{t}, J=7.3 \mathrm{~Hz}, 3 \mathrm{H}) .{ }^{13} \mathbf{C}$ NMR $\left(101 \mathrm{MHz}, \mathrm{CDCl}_{3}\right) \delta 167.5,150.0,141.0,128.3,127.1$, $125.5,124.4,60.7,29.2,26.4,26.1,25.5,17.6,15.0,14.5,14.3$. HRMS (TOF-MS ES ${ }^{+}$): $[\mathrm{M}+\mathrm{H}]^{+}$, calculated for $\mathrm{C}_{18} \mathrm{H}_{25} \mathrm{O}_{2}: 273.1855$; found $273.1816 .[\alpha]_{\mathrm{D}}{ }^{20}=+14.5(\mathrm{c}=0.48$, $\left.\mathrm{CHCl}_{3}\right)$. 
Note: The enantiomeric ratio of $\mathbf{3 e}$ could not be determined by HPLC or GC with the chiral columns tested (see chemical materials and methods). Therefore the same enantiomeric ratio as for $\mathbf{3 a}$ was assigned.

[1] This was done in order to remove a side product which was generated by addition of EtZnR to ethyl 2-(bromomethyl)acrylate. $\mathrm{R}_{\mathrm{f}}=0.50$ (Hexanes: EtOAc $=10: 1$ ).

\section{4-((1S,2S,3R)-3-ethyl-2-methyl-2-phenylcyclopropyl)butan-2-one (3f)}

\section{General procedure GP2:}

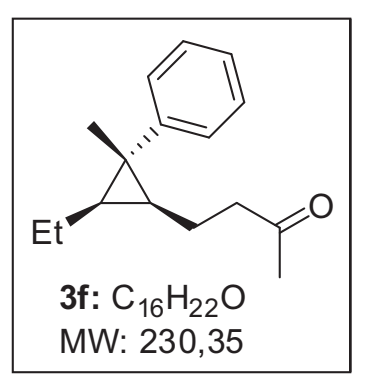

$\mathrm{Cu}\left(\mathrm{CH}_{3} \mathrm{CN}\right)_{4} \mathrm{PF}_{6}(11.2 \mathrm{mg}, 0.03 \mathrm{mmol}, 3 \mathrm{~mol} \%)$ and $(R)-\mathrm{DTBM}-$ SEGPHOS $^{\circledR}(38.9 \mathrm{mg}, 0.033 \mathrm{mmol}, 3.3 \mathrm{~mol} \%)$ were dissolved in THF $(4.0 \mathrm{~mL})$ under argon atmosphere. After stirring the pale pink solution for $1 \mathrm{~h}$ at $\mathrm{rt}$ the mixture was cooled to $0{ }^{\circ} \mathrm{C}$ and the cyclopropene substrate (130.2 mg, $1.0 \mathrm{mmol}, 1.0$ eq.) was added as a $0.5 \mathrm{M}$ solution in pentane. After 5 min $\mathrm{Et}_{2} \mathrm{Zn}(1.0 \mathrm{M}$ solution in hexanes; $1.2 \mathrm{~mL}, 1.2$ mmol, 1.2 eq.) was added dropwise at the wall of the flask over 1 min. After the reaction was stirred for $3 \mathrm{~h}$ at $0{ }^{\circ} \mathrm{C}$ the reaction vessel was cooled to $-40{ }^{\circ} \mathrm{C}$ and $\mathrm{CuCN} 2 \mathrm{LiCl}(0.5 \mathrm{M}$ in THF; $5.3 \mathrm{~mL}, 2.64 \mathrm{mmol}, 2.6$ eq.) was added followed by TMSCl $(675 \mu \mathrm{L}, 574 \mathrm{mg}, 5.3$ mmol, 5.3 eq.) and methyl vinyl ketone (240 $\mu \mathrm{L}, 201 \mathrm{mg}, 2.88 \mathrm{mmol}, 2.9$ eq.). After stirring for $1 \mathrm{~h}$ at $-40{ }^{\circ} \mathrm{C}$ the reaction was slowly warmed over $5 \mathrm{~h}$ to $\mathrm{rt}$ and stirring was continued for $4 \mathrm{~h}$ at $\mathrm{rt}$. The reaction was quenched with $\mathrm{HCl}(1 \mathrm{M}, 5 \mathrm{~mL})$ and stirred for $20 \mathrm{~min}$ at $\mathrm{rt}$. Then $\mathrm{NH}_{4} \mathrm{OH}$ was added until the $\mathrm{pH}$ was $\sim 9$. The blue reaction mixture was extracted with $\mathrm{Et}_{2} \mathrm{O}(3 \times 10 \mathrm{~mL})$ and the combined ethereal phases washed with dist. $\mathrm{H}_{2} \mathrm{O}(2 \times 10 \mathrm{~mL})$, dried over $\mathrm{Na}_{2} \mathrm{SO}_{4}$ and reduced in vacuo. The oily residue was purified by flash chromatography (hexanes: EtOAc $=20: 1)$ to obtain $\mathbf{3 f}$ as a colorless oil $\left(189 \mathrm{mg}, 0.82 \mathrm{mmol}, 82 \%\right.$ yield). $\mathrm{R}_{\mathrm{f}}=$ 0.24 (Hexanes: EtOAc $=20: 1$; stained with anisaldehyde).

${ }^{1} \mathbf{H}$ NMR $\left(400 \mathrm{MHz}, \mathrm{CDCl}_{3}\right) \delta 7.20-7.10(\mathrm{~m}, 4 \mathrm{H}), 7.05(\mathrm{~d}, J=6.9 \mathrm{~Hz}, 1 \mathrm{H}), 2.48(\mathrm{t}, J=7.7$ $\mathrm{Hz}, 2 \mathrm{H}), 2.06$ (s, 3H), $1.70-1.52(\mathrm{~m}, 2 \mathrm{H}), 1.45-1.25(\mathrm{~m}, 2 \mathrm{H}), 1.16(\mathrm{~s}, 3 \mathrm{H}), 0.97$ (t, $J=7.3$ $\mathrm{Hz}, 3 \mathrm{H}), 0.94-0.88$ (m, $2 \mathrm{H}) .{ }^{13} \mathbf{C}$ NMR $\left(101 \mathrm{MHz}, \mathrm{CDCl}_{3}\right) \delta 208.7,150.2,128.2,127.4$, 125.4, 44.2, 30.0, 29.1, 26.4 (2C's), 18.8, 17.4, 15.2, 14.5. HRMS (TOF-MS ES $\left.{ }^{+}\right):[\mathrm{M}+\mathrm{H}]^{+}$, calculated for $\mathrm{C}_{16} \mathrm{H}_{23} \mathrm{O}$ : 231.1749; found 231.1724. HPLC analysis (CHIRALCEL OD, 1\% IPA: $99 \%$ hexanes, $1.0 \mathrm{~mL} / \mathrm{min}, 254 \mathrm{~nm}, \mathrm{t}_{\mathrm{R} 1}=6.5 \mathrm{~min}, \mathrm{t}_{\mathrm{R} 2}=7.4 \mathrm{~min}$ ) indicated $93: 7 \mathrm{er}$. $[\alpha]_{\mathrm{D}}{ }^{20}$ $=+19.3\left(\mathrm{c}=0.48, \mathrm{CHCl}_{3}\right)$. 

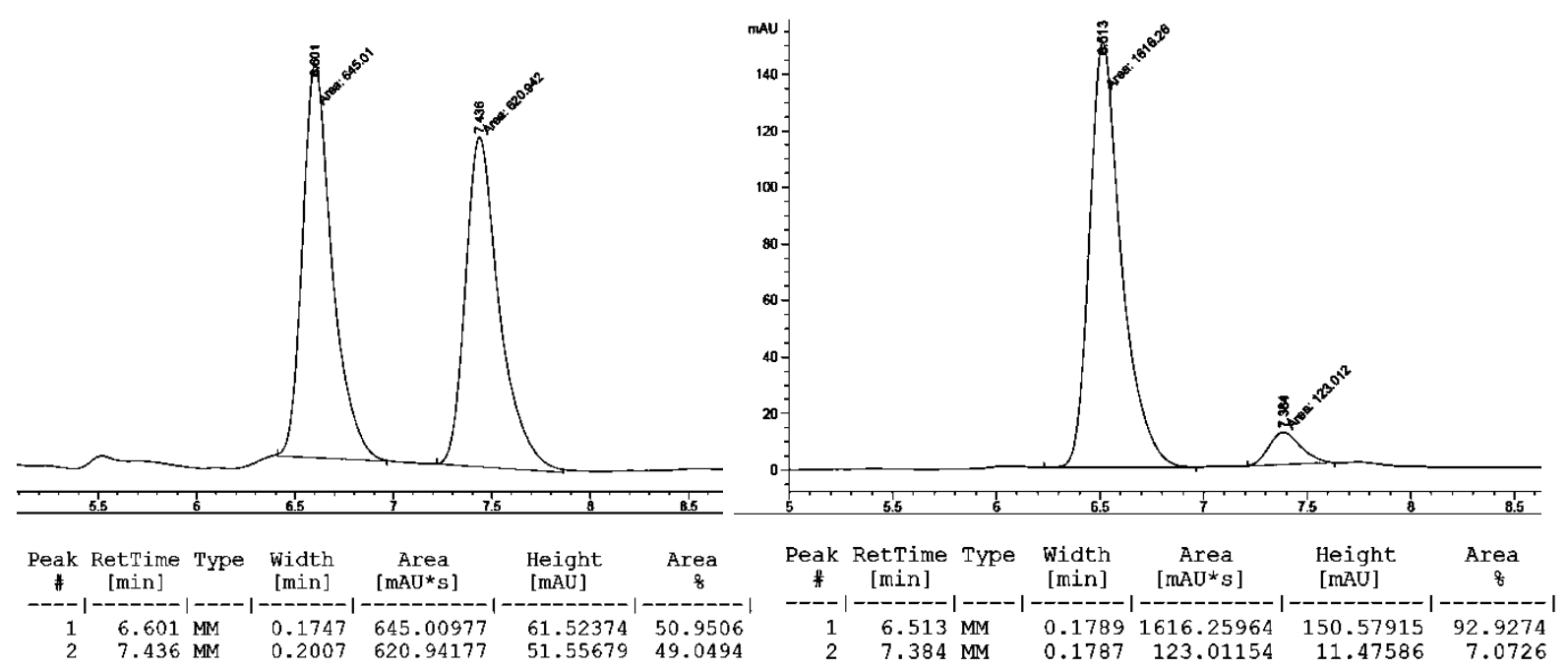

\section{4-((1S,2S,3R)-2,3-dimethyl-2-phenylcyclopropyl)butan-2-one (3g)}

\section{General procedure GP3:}

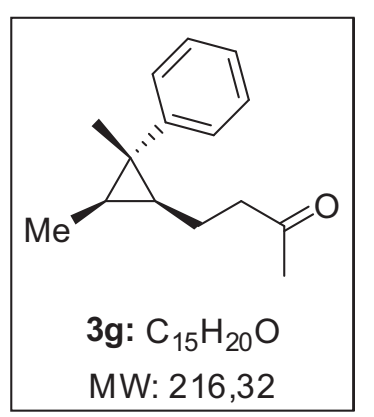

CuTC (9.5 mg, $0.038 \mathrm{mmol}, 5.0 \mathrm{~mol} \%)$ and $(R)-$ DTBM-SEGPHOS $^{\circledR}$ (48.3 mg, $0.041 \mathrm{mmol}, 5.5 \mathrm{~mol} \%$ ) were dissolved in $\mathrm{Et}_{2} \mathrm{O}(3.0 \mathrm{~mL})$ under argon atmosphere. After stirring the solution for $1 \mathrm{~h}$ at $\mathrm{rt}$ the mixture was cooled to $-78^{\circ} \mathrm{C}$ and the cyclopropene substrate $(1.5 \mathrm{~mL}$, $99.1 \mathrm{mg}, 0.75 \mathrm{mmol}, 1.0$ eq.; $0.5 \mathrm{M}$ solution in pentane) was added. After 5 min $\mathrm{Et}_{2} \mathrm{Zn}(0.9 \mathrm{~mL}, 0.9 \mathrm{mmol}, 1.2$ eq.; $1.0 \mathrm{M}$ solution in heptanes) was added dropwise at the wall of the flask. The reaction was stirred for $3 \mathrm{~h}$ at -78 ${ }^{\circ} \mathrm{C}$ then warmed to $-40{ }^{\circ} \mathrm{C}$ over $1 \mathrm{~h}$. $\mathrm{CuCN} 2 \mathrm{LiCl}(0.5 \mathrm{M}$ in THF; $4.0 \mathrm{~mL}, 1.99 \mathrm{mmol}, 2.64$ eq.) was added followed by $\mathrm{TMSCl}(506 \mu \mathrm{L}, 430 \mathrm{mg}, 4.0 \mathrm{mmol}, 5.3 \mathrm{eq}$.) and methyl vinyl ketone $\left(180 \mu \mathrm{L}, 151 \mathrm{mg}, 2.16 \mathrm{mmol}, 2.9\right.$ eq.). After stirring for $1 \mathrm{~h}$ at $-40{ }^{\circ} \mathrm{C}$ the reaction was slowly warmed over $5 \mathrm{~h}$ to $\mathrm{rt}$ and stirring was continued for $4 \mathrm{~h}$ at $\mathrm{rt}$. The reaction was quenched with $\mathrm{HCl}(1 \mathrm{M}, 5 \mathrm{~mL})$ and stirred for $20 \mathrm{~min}$ at $\mathrm{rt}$. Then $\mathrm{NH}_{4} \mathrm{OH}$ was added until the $\mathrm{pH}$ was $\sim 9$. The blue reaction mixture was extracted with $\mathrm{Et}_{2} \mathrm{O}(3 \times 10 \mathrm{~mL})$ and the combined ethereal phases washed with dist. $\mathrm{H}_{2} \mathrm{O}(2 \times 10 \mathrm{~mL})$, dried over $\mathrm{Na}_{2} \mathrm{SO}_{4}$ and reduced in vacuo. The oily residue was purified by flash chromatography (hexanes: EtOAc $=20: 1$ ) to obtain $\mathbf{3 g}$ as a colorless oil $\left(82 \mathrm{mg}, 0.38 \mathrm{mmol}, 51 \%\right.$ yield). $\mathrm{R}_{\mathrm{f}}=0.18$ (Hexanes: EtOAc $=$ 20:1; stained with anisaldehyde).

${ }^{1} \mathbf{H}$ NMR $\left(400 \mathrm{MHz}, \mathrm{CDCl}_{3}\right) \delta 7.23-7.11(\mathrm{~m}, 4 \mathrm{H}), 7.10-7.03(\mathrm{~m}, 1 \mathrm{H}), 2.49(\mathrm{t}, J=7.3 \mathrm{~Hz}$, 2H), $2.10(\mathrm{~s}, 3 \mathrm{H}), 1.62(\mathrm{dt}, J=7.8,7.5 \mathrm{~Hz}, 2 \mathrm{H}), 1.16(\mathrm{~s}, 3 \mathrm{H}), 1.12-1.05(\mathrm{~m}, 1 \mathrm{H}), 1.01(\mathrm{~d}, J$ $=6.2 \mathrm{~Hz}, 3 \mathrm{H}), 0.95-0.86(\mathrm{~m}, 1 \mathrm{H}) .{ }^{13} \mathbf{C} \mathbf{~ N M R}\left(101 \mathrm{MHz}, \mathrm{CDCl}_{3}\right) \delta 208.9,150.1,128.3$, 127.1, 125.4, 44.0, 30.0, 26.5, 25.9, 21.0, 18.6, 14.8, 8.3. HRMS (TOF-MS ES ${ }^{+}$): $[\mathrm{M}+\mathrm{H}]^{+}$, calculated for $\mathrm{C}_{15} \mathrm{H}_{21} \mathrm{O}: 217.1587$; found 217.1586. HPLC analysis (CHIRALCEL OD, 1\% 
IPA: 99\% hexanes, $1.0 \mathrm{~mL} / \mathrm{min}, 254 \mathrm{~nm}, \mathrm{t}_{\mathrm{R} 1}=6.88 \mathrm{~min}, \mathrm{t}_{\mathrm{R} 2}=7.68 \mathrm{~min}$ ) indicated $88: 12 \mathrm{er}$. $[\alpha]_{\mathbf{D}}{ }^{20}=+15.2\left(\mathrm{c}=0.58, \mathrm{CHCl}_{3}\right)$.

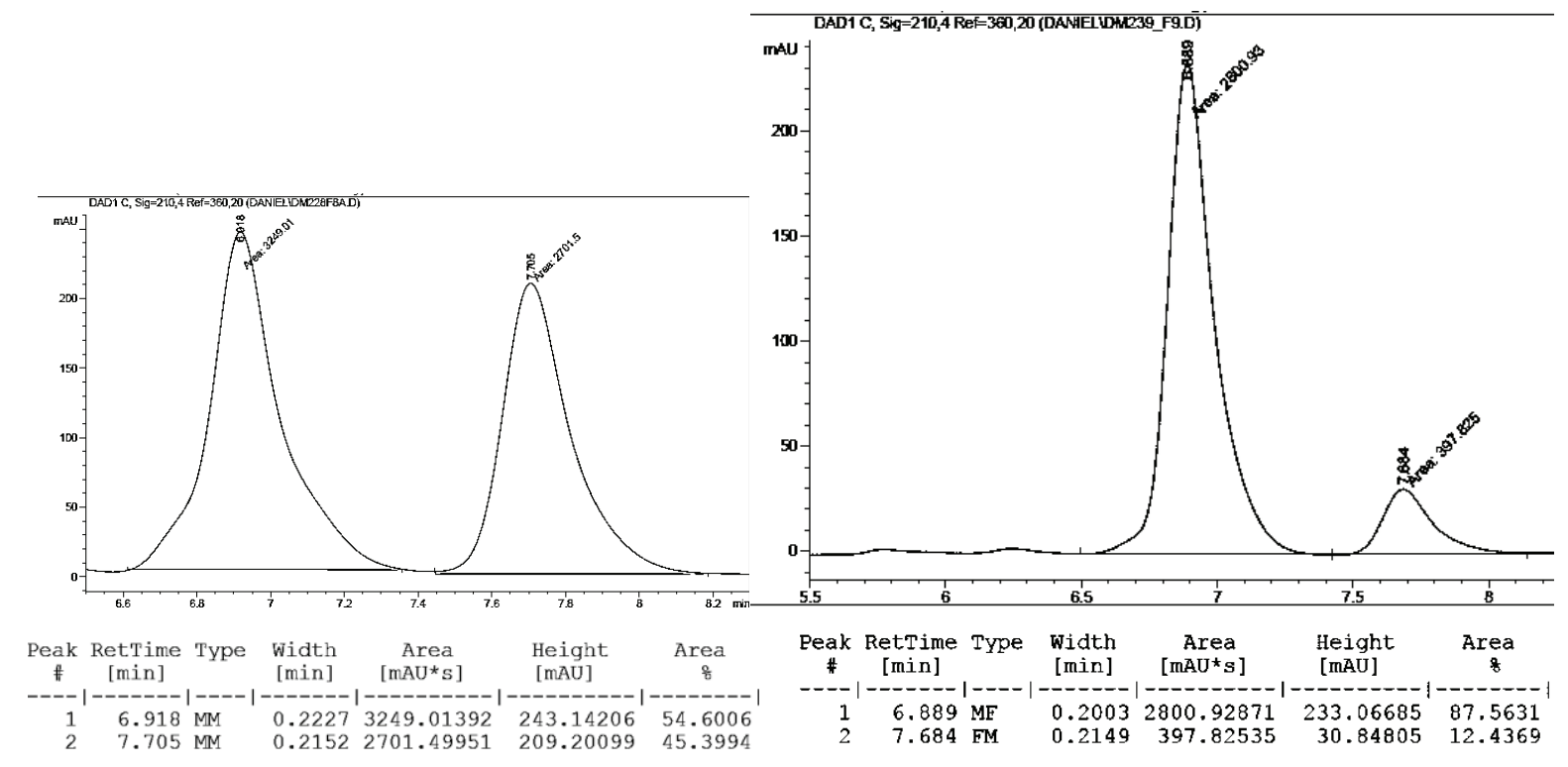

\section{4-((1S,2S,3R)-3-butyl-2-methyl-2-phenylcyclopropyl)butan-2-one (3h)}

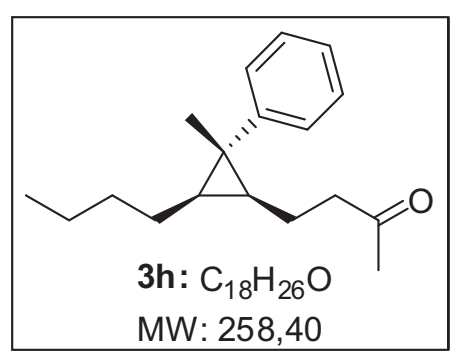

3h was prepared according to general procedure GP2 on 0.4 mmol scale. A $1.0 \mathrm{M}$ commercial solution of of $n \mathrm{Bu}_{2} \mathrm{Zn}$ in heptanes was used. The oily residue was purified by flash chromatography (hexanes: EtOAc $=20: 1$ ) to obtain $\mathbf{3 h}$ as a colorless oil (55 mg, $0.21 \mathrm{mmol}, 53 \%$ yield). $\mathrm{R}_{\mathrm{f}}=0.19$ (Hexanes: EtOAc $=20: 1$; stained with anisaldehyde).

${ }^{1} \mathbf{H}$ NMR $\left(400 \mathrm{MHz}, \mathrm{CDCl}_{3}\right) \delta 7.31-7.21(\mathrm{~m}, 4 \mathrm{H}), 7.18-7.12(\mathrm{~m}, 1 \mathrm{H}), 2.59(\mathrm{t}, J=7.7 \mathrm{~Hz}$, 2H), $2.19(\mathrm{~s}, 3 \mathrm{H}), 1.79-1.65(\mathrm{~m}, 2 \mathrm{H}), 1.49-1.33(\mathrm{~m}, 6 \mathrm{H}), 1.26(\mathrm{~s}, 3 \mathrm{H}), 1.09-0.98(\mathrm{~m}, 2 \mathrm{H})$, $0.94(\mathrm{t}, J=7.0 \mathrm{~Hz}, 3 \mathrm{H}) .{ }^{13} \mathbf{C}$ NMR $\left(101 \mathrm{MHz}, \mathrm{CDCl}_{3}\right) \delta 209.1,150.3,128.4,127.5,125.6$, 44.3, 32.6, 30.2, 27.4, 26.5, 26.4, 24.0, 23.0, 19.0, 15.4, 14.3. HRMS (TOF-MS ES ${ }^{+}$): $[\mathrm{M}+\mathrm{H}]^{+}$, calculated for $\mathrm{C}_{18} \mathrm{H}_{27} \mathrm{O}: 259.2056$; found 259.2050. HPLC analysis (CHIRALCEL OD, 1\% IPA: 99\% hexanes, $1.0 \mathrm{~mL} / \mathrm{min}, 254 \mathrm{~nm}, \mathrm{t}_{\mathrm{R} 1}=5.8 \mathrm{~min}, \mathrm{t}_{\mathrm{R} 2}=6.4 \mathrm{~min}$ ) indicated $86: 14 \operatorname{er}[\alpha]_{\mathbf{D}}{ }^{20}=+8.4\left(\mathrm{c}=0.40, \mathrm{CHCl}_{3}\right)$. 

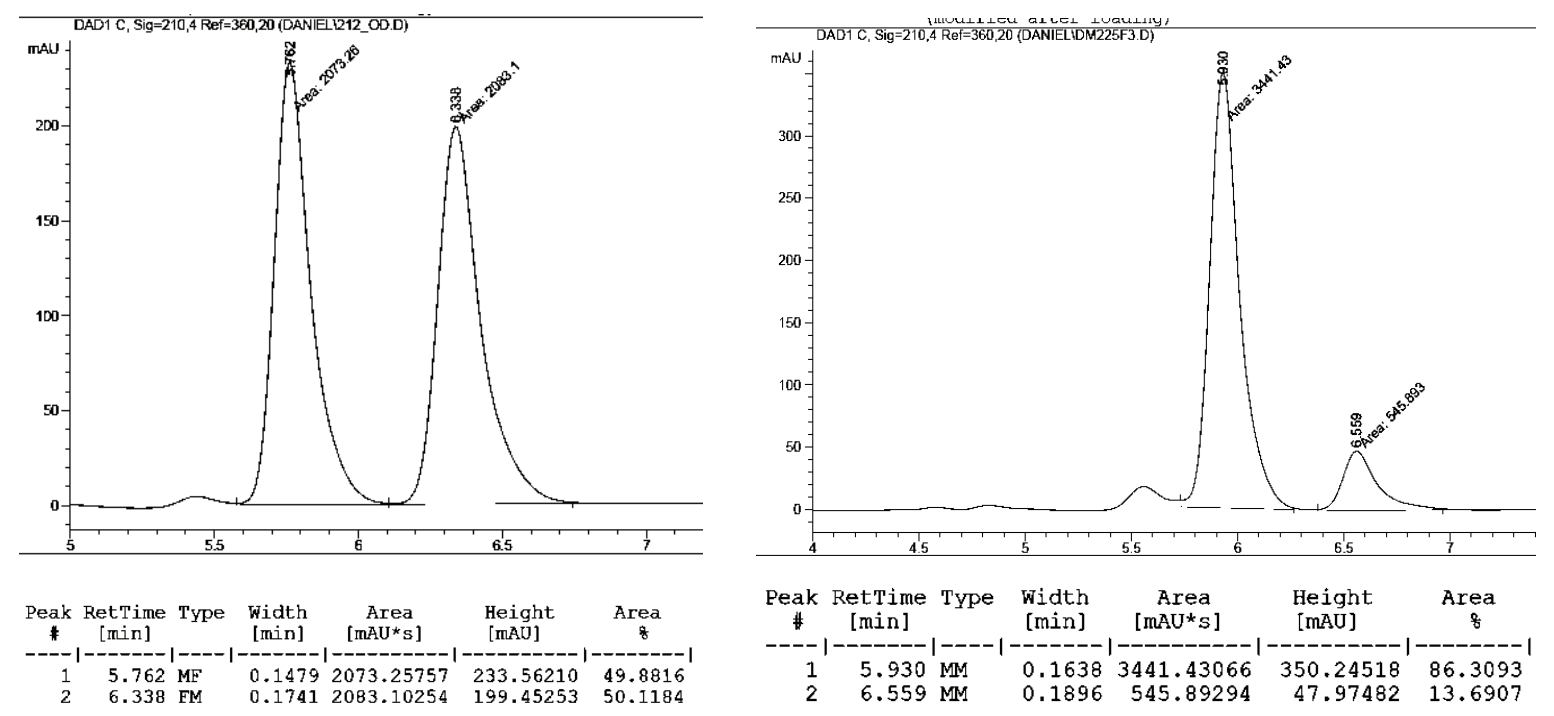

((1R,2S)-1-methylcyclopropane-1,2-diyl)dibenzene (3i)

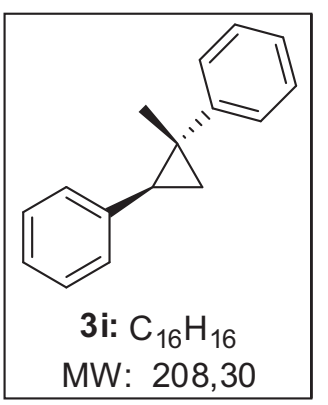

3i was prepared according to the general procedure GP3 on a $1.0 \mathrm{mmol}$ scale. A 1.0 M commercial solution of of commercially available $\mathrm{Ph}_{2} \mathrm{Zn}$ in THF was used. Flash chromatography (hexanes) of the crude product afforded $3 \mathbf{i}$ as a colorless oil (148 $\mathrm{mg}, 0.85 \mathrm{mmol}, 85 \%$ yield) and as a 93:7 mixture of diastereomers. $\mathrm{R}_{\mathrm{f}}=0.52$ (minor) and 0.57 (major); (hexanes; stained with phosphomolybdic acid). By column chromatographic purification a diastereomerically pure sample was obtained.

${ }^{1} \mathbf{H}$ NMR (400 MHz, $\mathrm{CDCl}_{3}$; major diastereomer) $\delta 7.40-7.26(\mathrm{~m}, 8 \mathrm{H}), 7.24-7.16(\mathrm{~m}, 2 \mathrm{H})$, $2.40(\mathrm{dd}, J=8.5,6.7 \mathrm{~Hz}, 1 \mathrm{H}), 1.44(\mathrm{dd}, J=8.7,5.1 \mathrm{~Hz}, 1 \mathrm{H}), 1.25-7.16(\mathrm{~m}, 1 \mathrm{H}), 1.11$ (s, $3 \mathrm{H}) .{ }^{13} \mathrm{C}$ NMR $\left(101 \mathrm{MHz}, \mathrm{CDCl}_{3}\right) \delta 148.0,139.2,129.3,128.5,128.2,127.0,126.1,125.9$, $31.5,27.1,21.1,18.8$. Spectral data consistent with reported data. ${ }^{[3]}$

HPLC analysis (CHIRALCEL OD, 1\% IPA: 99\% hexanes, $1.0 \mathrm{~mL} / \mathrm{min}, 254 \mathrm{~nm}, \mathrm{t}_{\mathrm{R} 1}=6.5$ $\left.\min , \mathrm{t}_{\mathrm{R} 2}=7.4 \mathrm{~min}\right)$ indicated $82: 18$ er. $[\alpha]_{\mathbf{D}}{ }^{20}=-118.9\left(\mathrm{c}=0.51, \mathrm{CHCl}_{3}\right)$. Lit. $^{[4]}[\alpha]_{\mathbf{D}}{ }^{20}=$ $-156.5\left(\mathrm{c}=1.08, \mathrm{CHCl}_{3} ; 95: 5 \mathrm{er}\right)$.
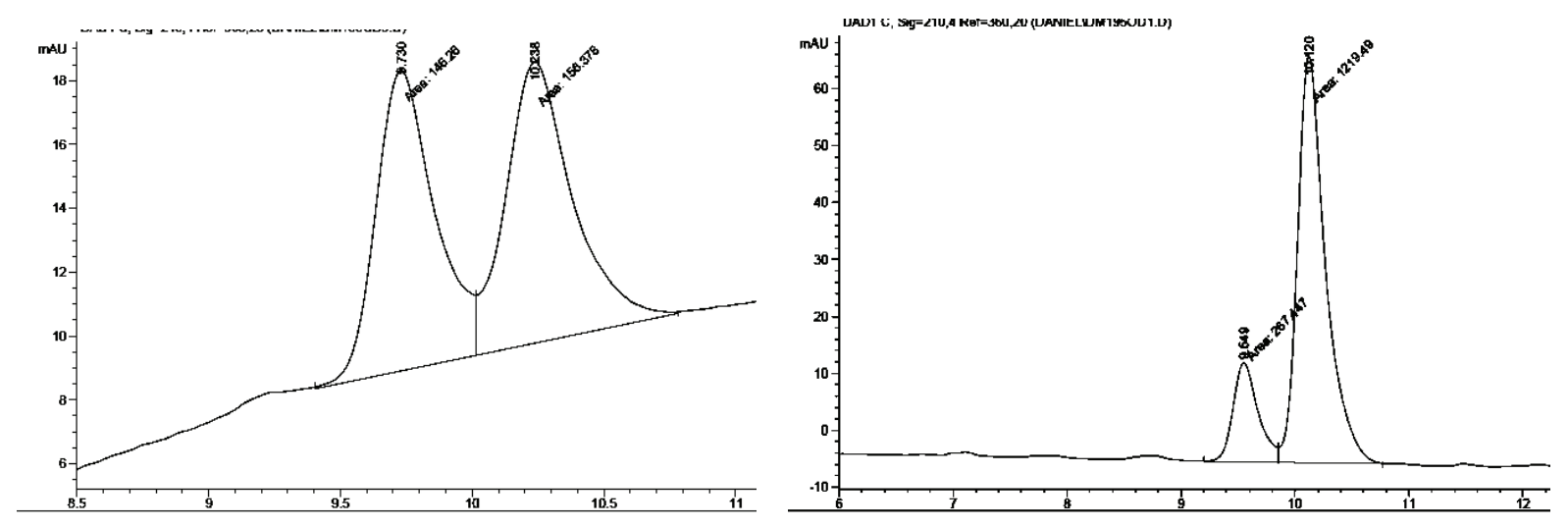


\begin{tabular}{|c|c|c|c|c|c|c|c|c|c|c|c|c|c|}
\hline Peak & $\begin{array}{l}\text { RetTime } \\
\text { [min] }\end{array}$ & Type & $\begin{array}{l}\text { Width } \\
\text { [min] }\end{array}$ & $\begin{array}{c}\text { Area } \\
{\left[\mathrm{mAU}^{\star} \mathrm{s}\right]}\end{array}$ & $\begin{array}{l}\text { Height } \\
\text { [mAU] }\end{array}$ & $\begin{array}{c}\text { Area } \\
\frac{8}{\delta}\end{array}$ & $\begin{array}{c}\text { Peak } \\
\#\end{array}$ & $\begin{array}{l}\text { RetTime } \\
\text { [min] }\end{array}$ & Type & $\begin{array}{l}\text { Width } \\
\text { [min] }\end{array}$ & $\begin{array}{c}\text { Area } \\
{\left[\mathrm{mAU}^{\star} \mathrm{S}\right]}\end{array}$ & $\begin{array}{l}\text { Height } \\
{[\mathrm{MAU}]}\end{array}$ & $\begin{array}{c}\text { Area } \\
8\end{array}$ \\
\hline$\frac{1}{2}$ & $\begin{array}{r}9.730 \\
10.238\end{array}$ & $\begin{array}{l}\text { MF } \\
\text { FM }\end{array}$ & $\begin{array}{l}0.2588 \\
0.2956\end{array}$ & & & $\begin{array}{l}48.3284 \\
51.6716\end{array}$ & $\begin{array}{l}1 \\
2\end{array}$ & $\begin{array}{r}9.549 \\
10.120\end{array}$ & $\begin{array}{l}\text { MF } \\
\text { FM }\end{array}$ & $\begin{array}{l}0.2566 \\
0.2849\end{array}$ & $\begin{array}{r}267.44739 \\
1219.49304\end{array}$ & $\begin{array}{l}17.36959 \\
71.33407\end{array}$ & $\begin{array}{l}17.9864 \\
82.0136\end{array}$ \\
\hline
\end{tabular}

\section{1-chloro-4-((1R,2R)-2-ethyl-1-methylcyclopropyl)benzene (3j)}

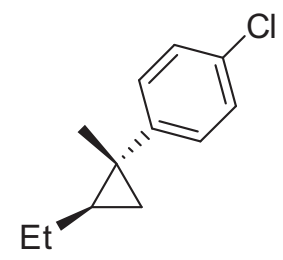

3j $\mathrm{C}_{12} \mathrm{H}_{15} \mathrm{Cl}$ MW: 194,70

Cyclopropane $\mathbf{3} \mathbf{j}$ was prepared according to general procedure GP1 (1.0 mmol scale). The catalyst loading was $5.0 \mathrm{~mol} \%$ (unoptimized conditions). After standard work-up the oily residue was purified by flash chromatography (hexanes) to obtain $\mathbf{3} \mathbf{j}$ as a colorless oil (185 $\mathrm{mg}, 0.95 \mathrm{mmol}$, 95\% yield). $\mathrm{R}_{\mathrm{f}}=0.85$ (Hexanes; stained with phosphomolybdic acid). ${ }^{1} \mathbf{H}$ NMR $\left(400 \mathrm{MHz}, \mathrm{CDCl}_{3}\right) \delta 7.28-7.25$ (m, 2H), $7.22-7.19(\mathrm{~m}, 2 \mathrm{H}), 1.61-1.46(\mathrm{~m}, 2 \mathrm{H}), 1.40(\mathrm{~s}, 3 \mathrm{H}), 1.11$ (t, $J=7.4 \mathrm{~Hz}, 3 \mathrm{H}), 1.06$ (dd, $J=8.9,4.4 \mathrm{~Hz}, 1 \mathrm{H}), 1.01-0.93(\mathrm{~m}, 1 \mathrm{H}), 0.44-0.41(\mathrm{~m}, 1 \mathrm{H})$. ${ }^{13} \mathbf{C}$ NMR $\left(101 \mathrm{MHz}, \mathrm{CDCl}_{3}\right) \delta 147.7,131.1,128.6,128.3,28.5,23.8,23.0,20.7,20.2$, 14.6. HRMS (TOF-MS ES ${ }^{+}$); $[\mathrm{M}+\mathrm{H}]^{+}$, calculated for $\mathrm{C}_{12} \mathrm{H}_{16} \mathrm{Cl}$ : 195.0941; found 195.0945. GC analysis $\left(\right.$ Cyclodex-B; 80-1-120; $\left.\mathrm{t}_{\mathrm{R} 1}=37.5 \mathrm{~min}, \mathrm{t}_{\mathrm{R} 2}=38.2 \mathrm{~min}\right)$ indicated $91: 9 \mathrm{er} .[\alpha]_{\mathbf{D}}{ }^{\mathbf{2 0}}=$ $-21.3\left(\mathrm{c}=0.84, \mathrm{CHCl}_{3}\right)$.
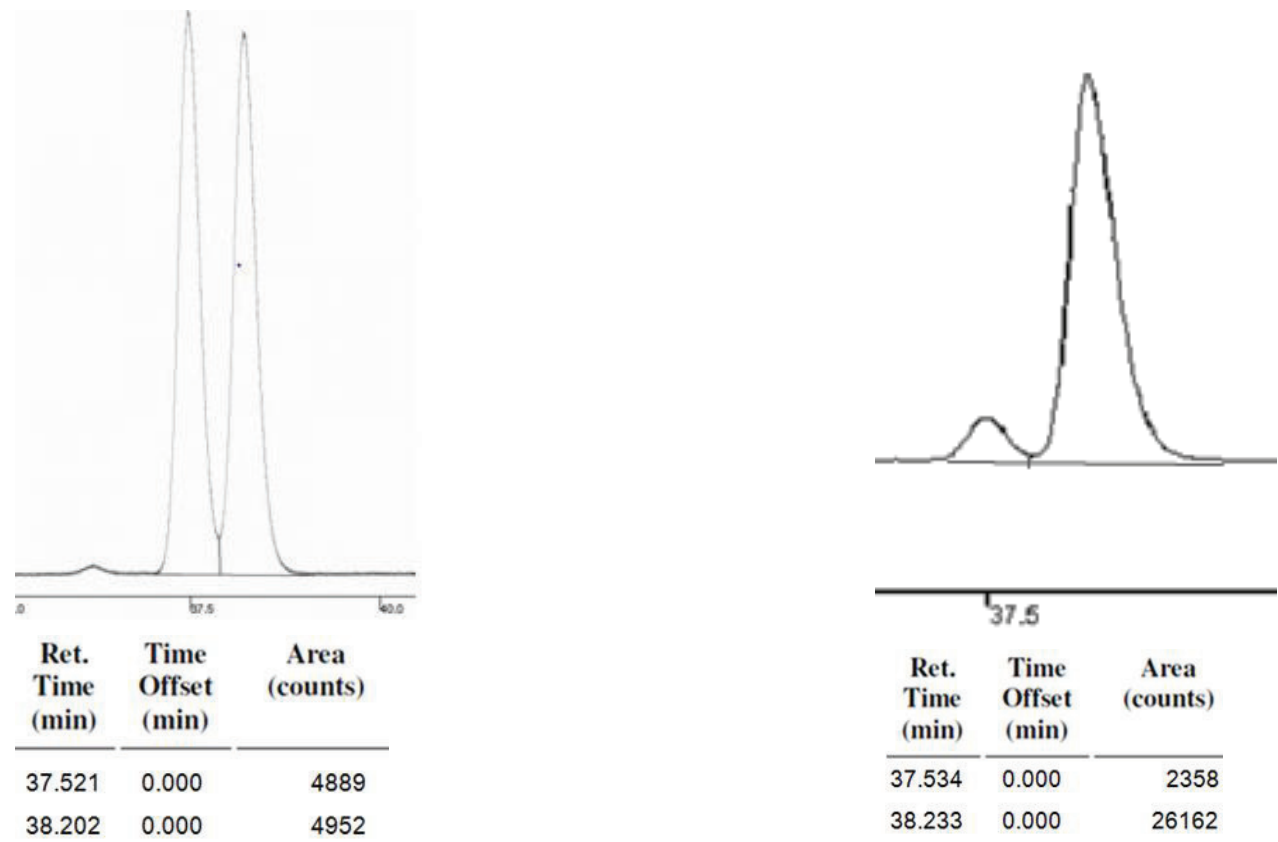


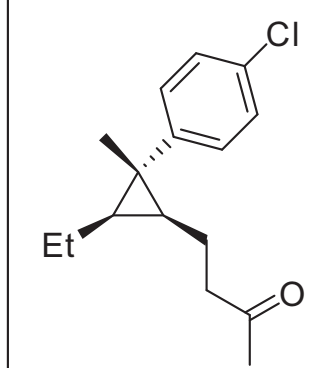

3k: $\mathrm{C}_{16} \mathrm{H}_{21} \mathrm{ClO}$ MW: 264,79

Ketone 3k was prepared according to general procedure GP2 (1.0 mmol scale). The catalyst loading was $5.0 \mathrm{~mol} \%$ (unoptimized conditions). After standard work-up the oily residue was purified by flash chromatography (hexanes: EtOAc $=20: 1$ ) to obtain $\mathbf{3 k}$ as a colorless oil (174 mg, $0.66 \mathrm{mmol}, 66 \%$ yield). $\mathrm{R}_{\mathrm{f}}=0.21$ (Hexanes: EtOAc $=20: 1 ;$ stained with anisaldehyde). ${ }^{1} \mathbf{H}$ NMR $(400 \mathrm{MHz}$, $\left.\mathrm{CDCl}_{3}\right) \delta 7.23-7.19(\mathrm{~m}, 2 \mathrm{H}), 7.16-7.11(\mathrm{~m}, 2 \mathrm{H}), 2.56(\mathrm{t}, J=7.6 \mathrm{~Hz}$, $2 \mathrm{H}), 2.17(\mathrm{~s}, 3 \mathrm{H}), 1.78-1.62(\mathrm{~m}, 2 \mathrm{H}), 1.54-1.32(\mathrm{~m}, 2 \mathrm{H}), 1.22(\mathrm{~s}$, $3 \mathrm{H}), 1.05(\mathrm{t}, J=7.3 \mathrm{~Hz}, 3 \mathrm{H}), 1.00-0.92(\mathrm{~m}, 2 \mathrm{H}) .{ }^{13} \mathbf{C} \mathbf{N M R}\left(101 \mathrm{MHz}, \mathrm{CDCl}_{3}\right) \delta 208.8$, 148.8, 131.1, 128.9, 128.4, 44.1, 30.1, 29.3, 26.6, 26.0, 18.4, 17.4, 15.1, 14.5. HRMS (TOFMS ES $\left.{ }^{+}\right):[\mathrm{M}+\mathrm{H}]^{+}$, calculated for $\mathrm{C}_{16} \mathrm{H}_{22} \mathrm{OCl}$ : 265.1359; found 265.1350. HPLC analysis (CHIRALCEL AD-H, 0.5\% IPA: 99.5\% hexanes, $1.0 \mathrm{~mL} / \mathrm{min}, 254 \mathrm{~nm}, \mathrm{t}_{\mathrm{R} 1}=7.0 \mathrm{~min}, \mathrm{t}_{\mathrm{R} 2}=$ $8.0 \mathrm{~min})$ indicated $91: 9 \mathrm{er} .[\alpha]_{\mathbf{D}}{ }^{20}=+18.7\left(\mathrm{c}=0.42, \mathrm{CHCl}_{3}\right)$.
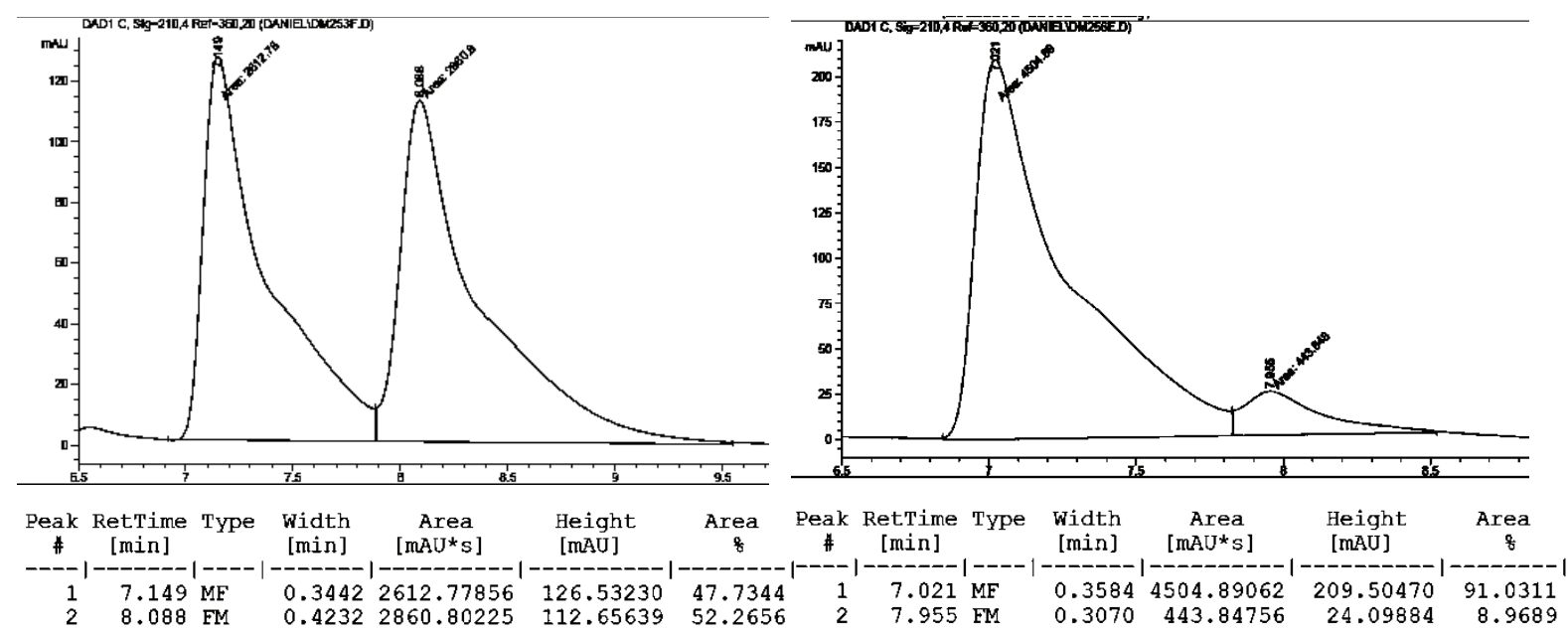

1-(4-((1R,2S,3R)-2-(4-chlorophenyl)-3-ethyl-2-methylcyclopropyl)phenyl)ethanone (3s)

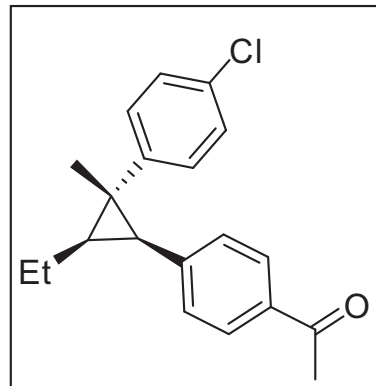

3s: $\mathrm{C}_{20} \mathrm{H}_{21} \mathrm{ClO}$ MW: 312,83 $\mathrm{Cu}\left(\mathrm{CH}_{3} \mathrm{CN}\right)_{4} \mathrm{PF}_{6}(3.8 \mathrm{mg}, 0.01 \mathrm{mmol}, 1.0 \mathrm{~mol} \%)$ and $(R)-\mathrm{DTBM}-$ SEGPHOS $^{\circledR}(13.0 \mathrm{mg}, 0.011 \mathrm{mmol}, 1.1 \mathrm{~mol} \%)$ were dissolved in THF $(4.0 \mathrm{~mL})$ under argon atmosphere. After stirring the pale pink solution for $1 \mathrm{~h}$ at $\mathrm{rt}$ the mixture was cooled to $0{ }^{\circ} \mathrm{C}$ and the cyclopropene substrate $(2.0 \mathrm{~mL}, 165 \mathrm{mg}, 1.0 \mathrm{mmol}, 1.0$ eq.) was

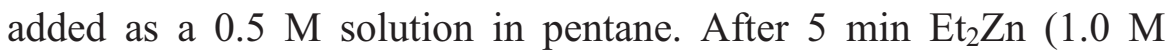
solution in hexanes; $1.2 \mathrm{~mL}, 1.2 \mathrm{mmol}, 1.2$ eq.) was added dropwise at the wall of the flask over $1 \mathrm{~min}$. After stirring the solution for $4 \mathrm{~h}$ 
at $0{ }^{\circ} \mathrm{C}$ a solution of $\mathrm{Pd}(\mathrm{OAc})_{2}(10.0 \mathrm{mg}, 0.04 \mathrm{mmol}, 0.04$ eq.) and SPhos (33 mg, $0.08 \mathrm{mmol}$, 0.08 eq.) in THF $(1.0 \mathrm{~mL})$ was added at $\mathrm{rt}$ followed by 4-bromoacetphenone (498 $\mathrm{mg}, 2.5$ mmol, 2.5 eq.). Stirring at $\mathrm{rt}$ was continued for $16 \mathrm{~h}$. Then the reaction mixture was treated with $\mathrm{HCl}(1 \mathrm{M}, 5 \mathrm{~mL})$ and extracted with $\mathrm{Et}_{2} \mathrm{O}(3 \times 15 \mathrm{~mL})$. The combined organic phases were dried over $\mathrm{Na}_{2} \mathrm{SO}_{4}$ and reduced in vacuo. The oily residue was heated to $250{ }^{\circ} \mathrm{C}$ at 1 mbar to remove 1-(4-ethylphenyl)ethanone and then purified by flash chromatography (hexanes / ethyl acetate $=20: 1)$ to obtain $3 \mathbf{s}$ as a colorless viscous oil $(235 \mathrm{mg}, 0.75 \mathrm{mmol}$, $75 \%$ yield). ${ }^{1} \mathbf{H}$ NMR $\left(400 \mathrm{MHz}, \mathrm{CDCl}_{3}\right) \delta 7.93(\mathrm{~d}, J=8.2 \mathrm{~Hz}, 2 \mathrm{H}), 7.38$ (apd, $J=8.0 \mathrm{~Hz}$, 2H), $7.34-7.27(\mathrm{~m}, 4 \mathrm{H}), 2.60(\mathrm{~s}, 3 \mathrm{H}), 2.41(\mathrm{~d}, J=9.4 \mathrm{~Hz}, 1 \mathrm{H}), 1.83-1.71(\mathrm{~m}, 1 \mathrm{H}), 1.49-$ $1.39(\mathrm{~m}, 1 \mathrm{H}), 1.28-1.21(\mathrm{~m}, 1 \mathrm{H}), 1.19(\mathrm{~s}, 3 \mathrm{H}), 1.14(\mathrm{t}, J=7.0 \mathrm{~Hz}, 3 \mathrm{H}) .{ }^{13} \mathbf{C}$ NMR $(101$ $\left.\mathrm{MHz}, \mathrm{CDCl}_{3}\right) \delta 197.9,147.8,143.6,135.3,131.7,131.1,129.1,128.6,128.4,31.8,31.1,28.5$, 26.6, 19.5, 17.6, 14.6. $\mathrm{R}_{\mathrm{f}}=0.53$ (Hexanes: EtOAc $=10: 1$; stained with anisaldehyde). HRMS (APCI): $[\mathrm{M}+\mathrm{H}]^{+}$, calculated for $\mathrm{C}_{20} \mathrm{H}_{22} \mathrm{ClO}$ : 313.1354; found 313.1360. HPLC analysis (CHIRALCEL OD, 1\% IPA: 99\% hexanes, $1.0 \mathrm{~mL} / \mathrm{min}, 254 \mathrm{~nm}, \mathrm{t}_{\mathrm{R} 1}=7.9 \mathrm{~min}, \mathrm{t}_{\mathrm{R} 2}=8.9 \mathrm{~min}$ ) indicated 91:9 er. $[\alpha]_{\mathbf{D}}{ }^{\mathbf{2 0}}=+166.9\left(\mathrm{c}=0.60, \mathrm{CHCl}_{3}\right)$.
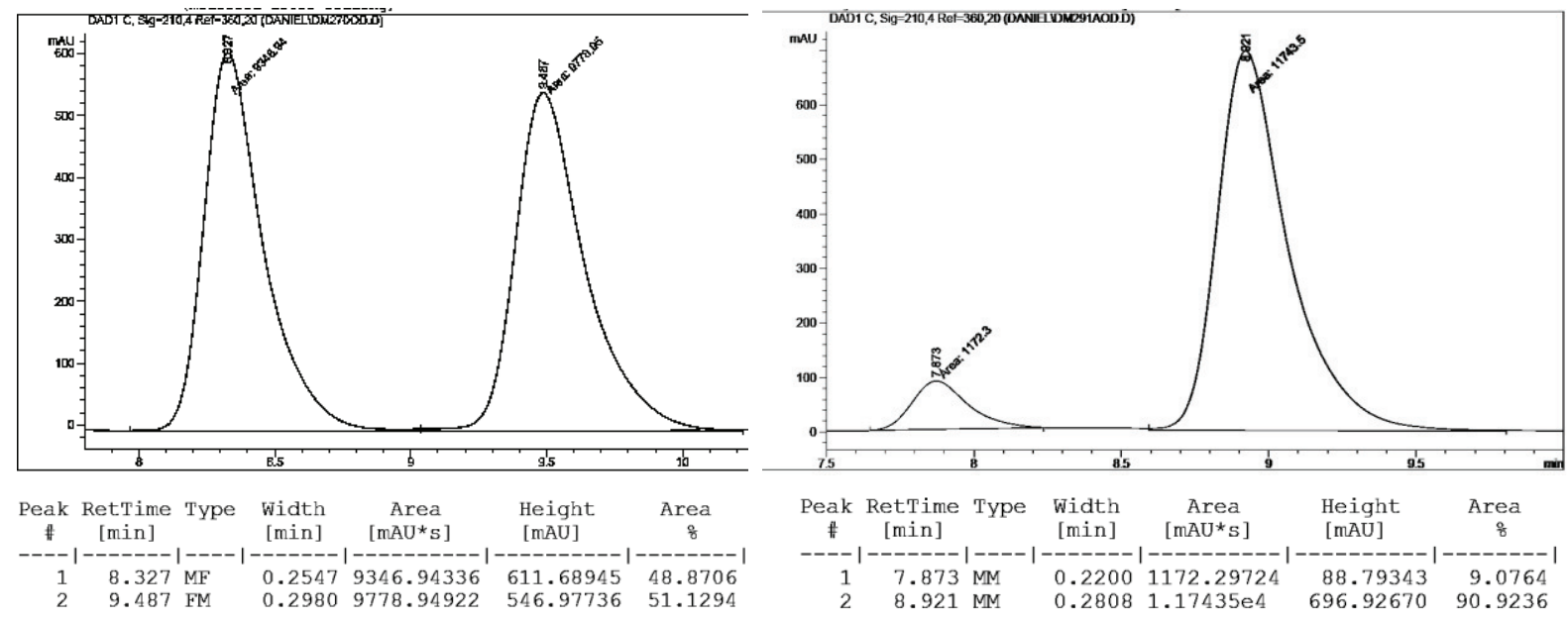

\section{4-((1R,2S,3R)-2-(4-chlorophenyl)-3-ethyl-2-methylcyclopropyl)benzaldehyde (3t)}

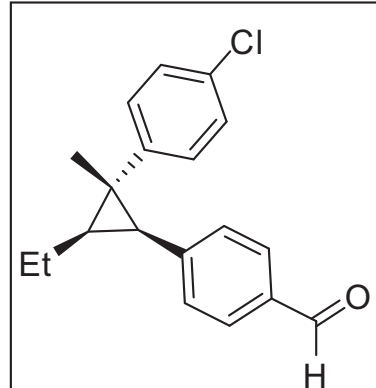

3t: $\mathrm{C}_{19} \mathrm{H}_{19} \mathrm{ClO}$ MW: 298,81
$\mathrm{Cu}\left(\mathrm{CH}_{3} \mathrm{CN}\right)_{4} \mathrm{PF}_{6}(3.8 \mathrm{mg}, 0.01 \mathrm{mmol}, 0.5 \mathrm{~mol} \%)$ and $(R)-\mathrm{DTBM}-$ SEGPHOS $^{\circledR}(13.0 \mathrm{mg}, 0.011 \mathrm{mmol}, 0.55 \mathrm{~mol} \%)$ were dissolved in THF $(8.0 \mathrm{~mL})$ under argon atmosphere. After stirring the pale pink solution for $1 \mathrm{~h}$ at $\mathrm{rt}$ the mixture was cooled to $0{ }^{\circ} \mathrm{C}$ and the cyclopropene substrate $(4.0 \mathrm{~mL}, 329.3 \mathrm{mg}, 2.0 \mathrm{mmol}, 1.0$ eq.) was

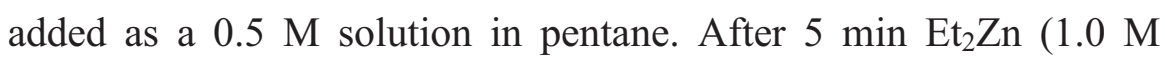
solution in hexanes; $2.4 \mathrm{~mL}, 2.4 \mathrm{mmol}, 1.2$ eq.) was added dropwise 
at the wall of the flask over $1 \mathrm{~min}$. After stirring the solution for $5 \mathrm{~h}$ at $0{ }^{\circ} \mathrm{C}$ a solution of $\mathrm{Pd}(\mathrm{OAc})_{2}$ (20.0 mg, $0.08 \mathrm{mmol}, 0.04$ eq.) and SPhos (66 mg, $0.16 \mathrm{mmol}, 0.08$ eq.) in THF $(2.0 \mathrm{~mL})$ was added at $\mathrm{rt}$ followed by 4-Bromobenzaldehyde (925 mg, $5.0 \mathrm{mmol}, 2.5$ eq.). Stirring at $\mathrm{rt}$ was continued for $16 \mathrm{~h}$. Then the reaction mixture was treated with $\mathrm{HCl}(1 \mathrm{M}, 5$ $\mathrm{mL})$ and extracted with $\mathrm{Et}_{2} \mathrm{O}(3 \times 15 \mathrm{~mL})$. The combined organic phases were dried over $\mathrm{Na}_{2} \mathrm{SO}_{4}$ and reduced in vacuo. The oily residue was heated to $250{ }^{\circ} \mathrm{C}$ at $1 \mathrm{mbar}$ to remove 4ethylbenzaldehyde and then purified by flash chromatography (hexanes / ethyl acetate $=20: 1$ ) to obtain $3 \mathrm{t}$ as a colorless oil (523 $\mathrm{mg}, 1.75 \mathrm{mmol}, 88 \%$ yield).

${ }^{1} \mathbf{H}$ NMR $\left(400 \mathrm{MHz}, \mathrm{CDCl}_{3}\right) \delta 10.00(\mathrm{~s}, 1 \mathrm{H}), 7.85$ (apd, $\left.J=8.1 \mathrm{~Hz}, 2 \mathrm{H}\right), 7.46(\mathrm{apd}, J=7.9$ Hz, 2H), $7.35-7.27$ (m, 4H), 2.43 (d, $J=9.4 \mathrm{~Hz}, 1 \mathrm{H}), 1.84-1.72(\mathrm{~m}, 1 \mathrm{H}), 1.52-1.72$ (m, 1H), $1.30-1.21(\mathrm{~m}, 1 \mathrm{H}), 1.19(\mathrm{~s}, 3 \mathrm{H}), 1.15$ (t, $J=7.0 \mathrm{~Hz}, 3 \mathrm{H}) .{ }^{13} \mathbf{C} \mathbf{N M R}\left(101 \mathrm{MHz}, \mathrm{CDCl}_{3}\right)$ $\delta 192.1,147.7,145.3,134.7,131.7,131.5,129.8,129.1,128.7,32.0,31.2,28.7,19.5,17.7$, 14.6. HRMS (APCI): $[\mathrm{M}+\mathrm{H}]^{+}$, calculated for $\mathrm{C}_{19} \mathrm{H}_{20} \mathrm{ClO}$ : 299.1197; found 299.1261. $\mathrm{R}_{\mathrm{f}}=$ 0.54 (Hexanes: EtOAc $=10: 1$; stained with $\left.\mathrm{KMnO}_{4}\right)$. HPLC analysis $(\mathrm{CHIRALCEL} \mathrm{AD-H}$, 1\% IPA: 99\% hexanes, $1.0 \mathrm{~mL} / \mathrm{min}, 254 \mathrm{~nm}, \mathrm{t}_{\mathrm{R} 1}=7.9 \mathrm{~min}, \mathrm{t}_{\mathrm{R} 2}=10.0 \mathrm{~min}$ ) indicated $91: 9 \mathrm{er}$. $[\alpha]_{\mathbf{D}}{ }^{20}=+178.4\left(\mathrm{c}=0.84, \mathrm{CHCl}_{3}\right)$.
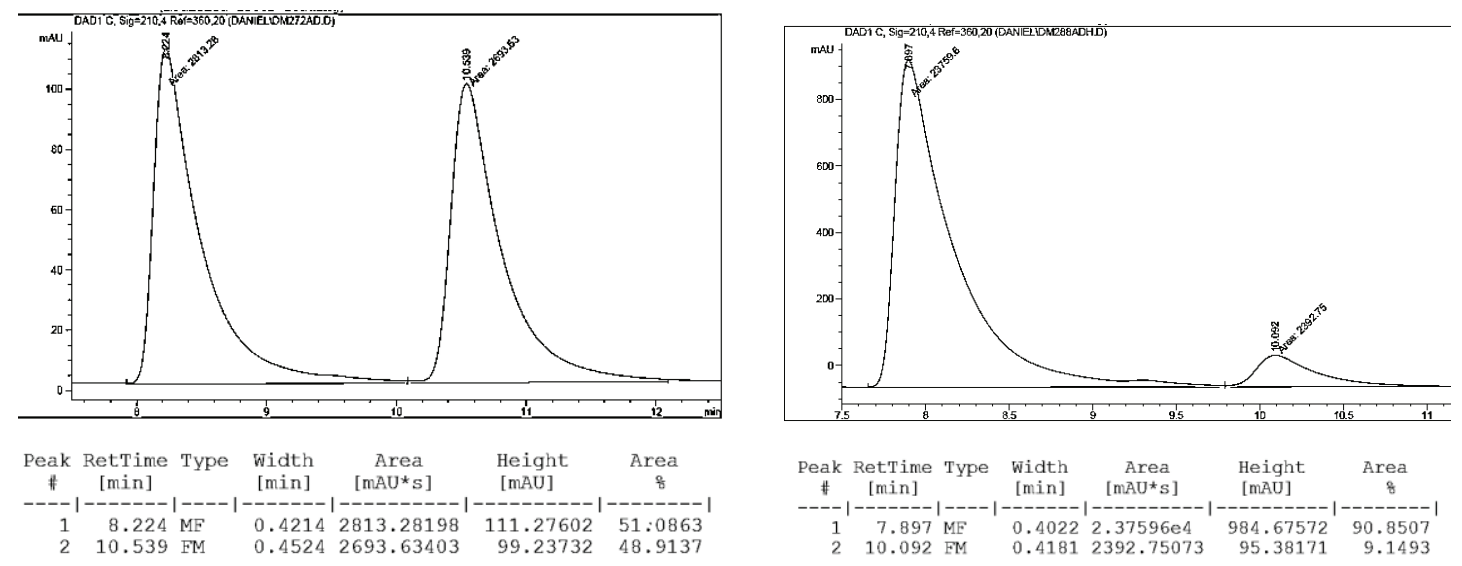

\section{4-((1S,2S,3R)-3-ethyl-2-(4-methoxyphenyl)-2-methylcyclopropyl)butan-2-one (3I)}

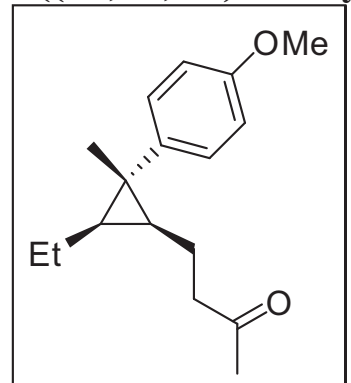

3I: $\mathrm{C}_{17} \mathrm{H}_{24} \mathrm{O}_{2}$ MW: 260,37
Ketone 3a was prepared according to general procedure GP2 (1.0 mmol scale). The catalyst loading was $5.0 \mathrm{~mol} \%$ (unoptimized conditions). After standard work-up the oily residue was purified by flash chromatography (hexanes: EtOAc $=20: 1$ ) to obtain 31 as a colorless oil (146 mg, $0.56 \mathrm{mmol}, 56 \%$ yield). $\mathrm{R}_{\mathrm{f}}=0.21$ (Hexanes: EtOAc $=20: 1 ;$ stained with anisaldehyde). ${ }^{1} \mathbf{H}$ NMR $(400 \mathrm{MHz}$, $\left.\mathrm{CDCl}_{3}\right) \delta 7.17-7.13(\mathrm{~m}, 2 \mathrm{H}), 6.85-6.79(\mathrm{~m}, 2 \mathrm{H}), 3.78(\mathrm{~s}, 3 \mathrm{H}), 2.58$ 
(t, $J=7.7 \mathrm{~Hz}, 2 \mathrm{H}), 2.18(\mathrm{~s}, 3 \mathrm{H}), 1.83-1.62(\mathrm{~m}, 2 \mathrm{H}), 1.54-1.33(\mathrm{~m}, 2 \mathrm{H}), 1.22(\mathrm{~s}, 3 \mathrm{H}), 1.06$ $(\mathrm{t}, J=7.3 \mathrm{~Hz}, 3 \mathrm{H}), 1.01-0.91(\mathrm{~m}, 2 \mathrm{H}) .{ }^{13} \mathrm{C} \mathrm{NMR}\left(101 \mathrm{MHz}, \mathrm{CDCl}_{3}\right) \delta 209.0,157.5,142.7$, 128.6, 113.7, 55.3, 44.3, 30.0, 28.9, 26.3, 25.9, 18.9, 17.5, 15.6, 14.1. HRMS (TOF-MS $\left.\mathrm{ES}^{+}\right):[\mathrm{M}+\mathrm{H}]^{+}$, calculated for $\mathrm{C}_{17} \mathrm{H}_{25} \mathrm{O}_{2}:$ 261.1855; found 261.1850. HPLC analysis (CHIRALCEL OD, 0.5\% IPA: 99.5\% hexanes, $1.0 \mathrm{~mL} / \mathrm{min}, 254 \mathrm{~nm}, \mathrm{t}_{\mathrm{R} 1}=9.5 \mathrm{~min}, \mathrm{t}_{\mathrm{R} 2}=10.2$ min) indicated 90:10 er. $[\alpha]_{\mathbf{D}}{ }^{20}=+18.9\left(\mathrm{c}=0.88, \mathrm{CHCl}_{3}\right)$.
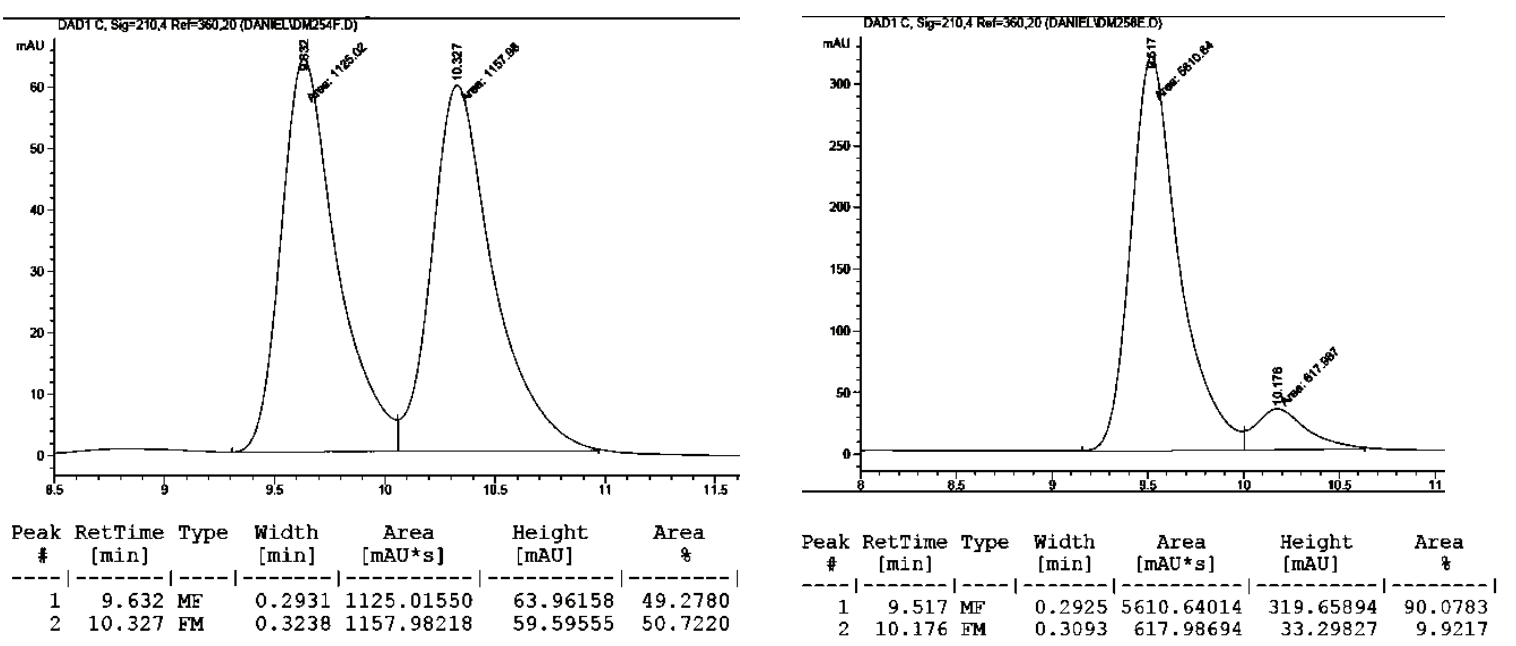

\section{4-((1S,2S,3R)-3-ethyl-2-isopropyl-2-phenylcyclopropyl)butan-2-one (3m)}

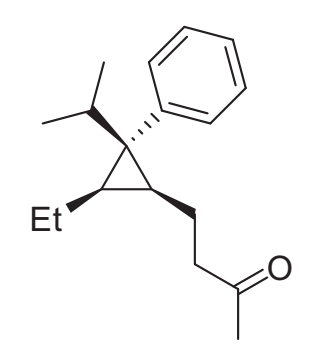

3m: $\mathrm{C}_{18} \mathrm{H}_{26} \mathrm{O}$ MW: 260,37

Ketone $\mathbf{3 m}$ was prepared according to general procedure GP2 (1.0 mmol scale). The catalyst loading was $5.0 \mathrm{~mol} \%$ (unoptimized conditions). After standard work-up the oily residue was purified by flash chromatography (hexanes: EtOAc $=20: 1$ ) to obtain $\mathbf{3 m}$ as a colorless oil (167 mg, $0.65 \mathrm{mmol}, 65 \%$ yield). $\mathrm{R}_{\mathrm{f}}=0.32$ (Hexanes: EtOAc $=20: 1 ;$ stained with anisaldehyde).

${ }^{1} \mathbf{H}$ NMR $\left(400 \mathrm{MHz}, \mathrm{CDCl}_{3}\right) \delta{ }^{1} \mathrm{H}$ NMR $\left(400 \mathrm{MHz}, \mathrm{CDCl}_{3}\right) \delta 7.25-$ $7.23(\mathrm{~m}, 4 \mathrm{H}), 7.19-7.14(\mathrm{~m}, 1 \mathrm{H}), 2.62(\mathrm{t}, J=7.4 \mathrm{~Hz}, 2 \mathrm{H}), 2.21-2.14(\mathrm{~m}, 3 \mathrm{H}), 2.04-1.89$ (m, 1H), $1.78-1.70(\mathrm{~m}, 1 \mathrm{H}), 1.69-1.59(\mathrm{~m}, 2 \mathrm{H}), 1.48-1.35(\mathrm{~m}, 1 \mathrm{H}), 1.11(\mathrm{t}, J=7.4 \mathrm{~Hz}$, $3 \mathrm{H}), 0.99-0.91(\mathrm{~m}, 2 \mathrm{H}), 0.85(\mathrm{~m}, 6 \mathrm{H}) .{ }^{13} \mathrm{C} \mathrm{NMR}\left(101 \mathrm{MHz}, \mathrm{CDCl}_{3}\right) \delta 208.9,145.4,131.8$, 127.3, 125.8, 44.7, 37.2, 30.1, 28.8, 26.7, 26.1, 20.8, 20.6, 18.1, 16.7, 15.2. HRMS (TOF-MS $\left.\mathrm{ES}^{+}\right):[\mathrm{M}+\mathrm{H}]^{+}$, calculated for $\mathrm{C}_{18} \mathrm{H}_{27} \mathrm{O}: 259.2062$; found 259.2060.

HPLC analysis (CHIRALCEL OD, 1\% IPA: 99\% hexanes, $1.0 \mathrm{~mL} / \mathrm{min}, 254 \mathrm{~nm}, \mathrm{t}_{\mathrm{R} 1}=6.0$ $\left.\min , t_{\mathrm{R} 2}=8.0 \mathrm{~min}\right)$ indicated 99.7:0.3 er. $[\alpha]_{\mathbf{D}}{ }^{20}=+16.9\left(\mathrm{c}=1.08, \mathrm{CHCl}_{3}\right)$. 


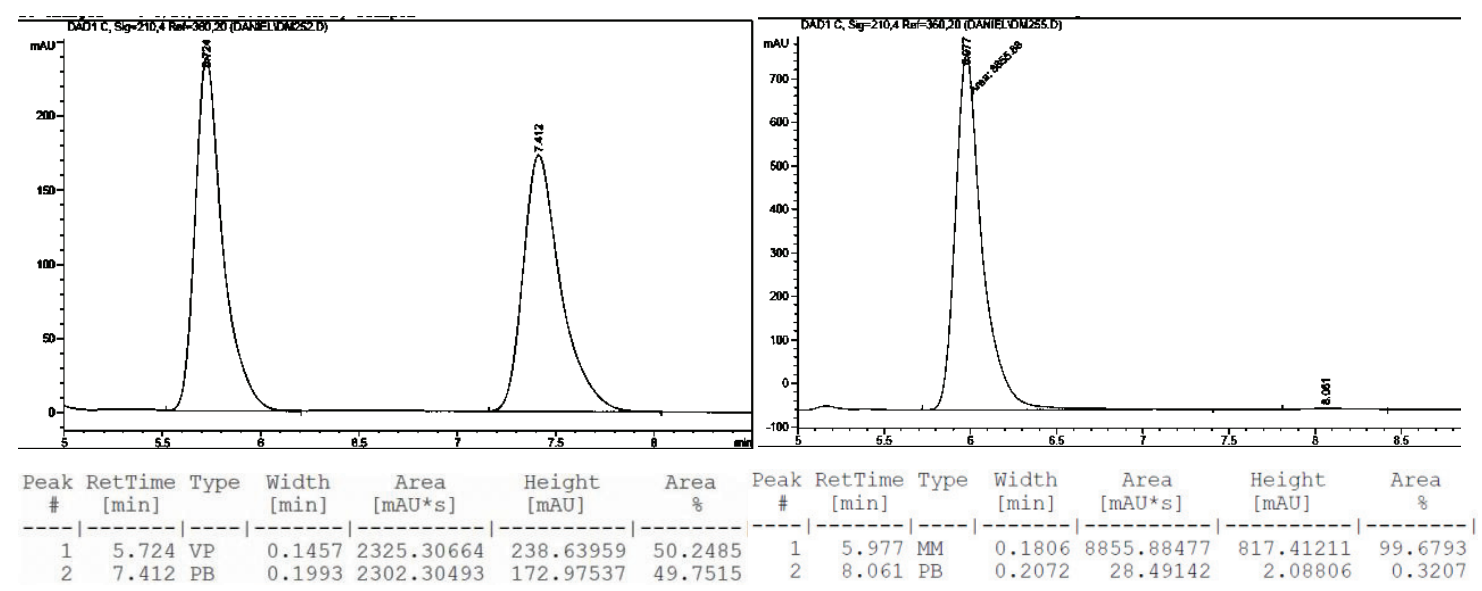

\section{4-((1S,2R,3S)-2-ethyl-3',4'-dihydro-2'H-spiro[cyclopropane-1,1'-naphthalene]-3-}

\section{yl)butan-2-one (3n)}

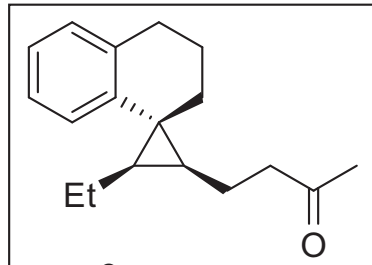

3n: $\mathrm{C}_{18} \mathrm{H}_{24} \mathrm{O}$ MW: 256,38

Ketone 3n was prepared according to general procedure GP2 (1.0 mmol scale). The catalyst loading was $5.0 \mathrm{~mol} \%$ (unoptimized conditions). After standard work-up the oily residue was purified by flash chromatography (hexanes: EtOAc $=20: 1$ ) to obtain $\mathbf{3 n}$ as a colorless viscous oil (165 mg, $0.64 \mathrm{mmol}, 64 \%$ yield). $\mathrm{R}_{\mathrm{f}}=0.25$ (Hexanes: EtOAc $=20: 1$; stained with anisaldehyde).

${ }^{1}$ H NMR $\left(400 \mathrm{MHz} \mathrm{CDCl}_{3}\right) \delta 7.16-7.10(\mathrm{~m}, 1 \mathrm{H}), 7.09-7.03(\mathrm{~m}, 2 \mathrm{H}), 6.72(\mathrm{~d}, J=7.9 \mathrm{~Hz}$, $1 \mathrm{H}), 2.88(\mathrm{t}, J=6.2 \mathrm{~Hz}, 2 \mathrm{H}), 2.57(\mathrm{dd}, J=9.7,5.7 \mathrm{~Hz}, 2 \mathrm{H}), 2.17(\mathrm{~s}, 3 \mathrm{H}), 1.92-1.82(\mathrm{~m}, 2 \mathrm{H})$, $1.79-1.69(\mathrm{~m}, 4 \mathrm{H}), 1.58-1.40(\mathrm{~m}, 2 \mathrm{H}), 1.19-1.10(\mathrm{~m}, 2 \mathrm{H}), 1.05(\mathrm{t}, J=7.4 \mathrm{~Hz}, 3 \mathrm{H}) .{ }^{13} \mathbf{C}$ NMR $\left(101 \mathrm{MHz}, \mathrm{CDCl}_{3}\right) \delta 208.7,143.8,137.0,128.6,126.2,124.3,121.7,44.1,34.2,31.4$, 31.0, 30.0, 23.6, 22.8, 18.4, 17.3, 14.6. $\mathrm{R}_{\mathrm{f}}=0.25$ (Hexanes: EtOAc $=20: 1$; stained with anisaldehyde). HRMS (TOF-MS $\mathrm{ES}^{+}$): $[\mathrm{M}+\mathrm{H}]^{+}$, calculated for $\mathrm{C}_{18} \mathrm{H}_{25} \mathrm{O}: 257.19055$ found 257.19049. HPLC analysis (CHIRALCEL OD, 1\% IPA: 99\% hexanes, $1.0 \mathrm{~mL} / \mathrm{min}, 254 \mathrm{~nm}$, $\left.t_{\mathrm{R} 1}=7.0 \mathrm{~min}, \mathrm{t}_{\mathrm{R} 2}=10.5 \mathrm{~min}\right)$ indicated 94:6 er. $[\alpha]_{\mathbf{D}}{ }^{20}=+41.1\left(\mathrm{c}=0.59, \mathrm{CHCl}_{3}\right)$.
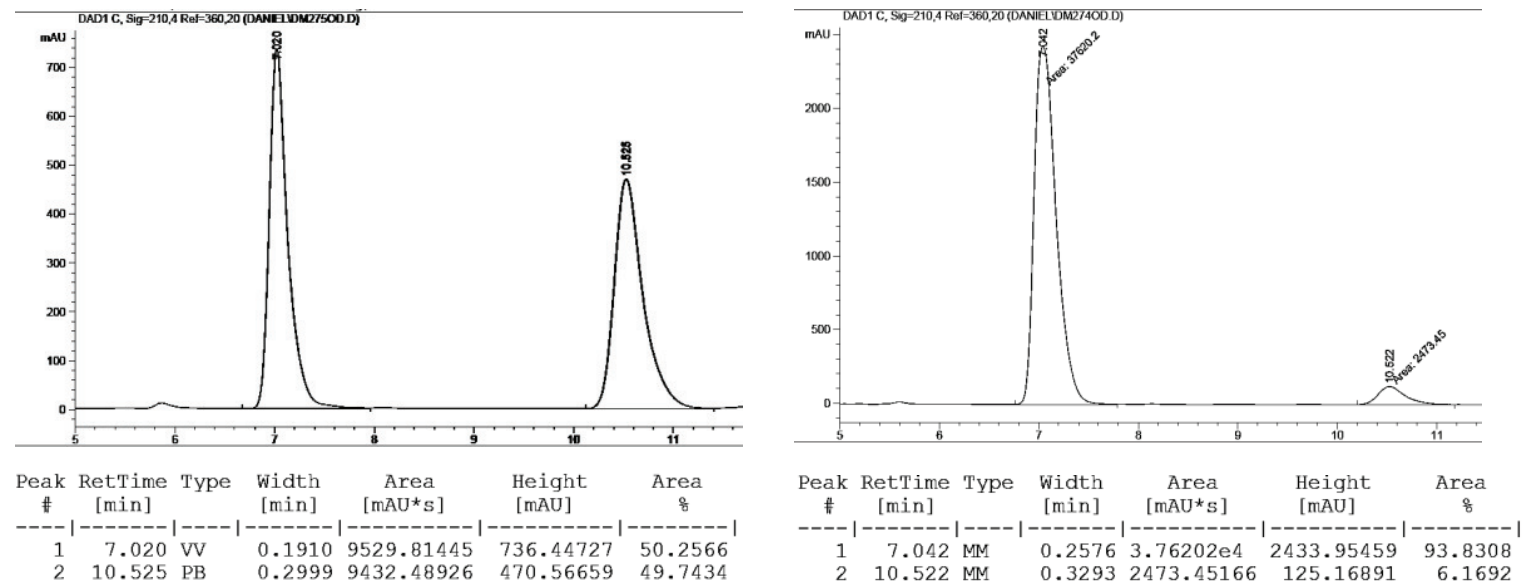


\section{(((1S,2R)-2-ethyl-1-methylcyclopropyl)methyl)benzene (3o)}

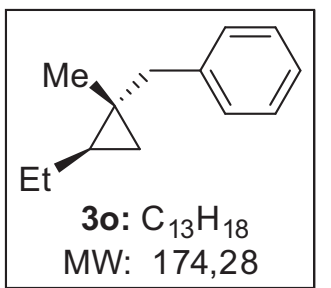

CuTC (9.5 mg, $0.05 \mathrm{mmol}, 5.0 \mathrm{~mol} \%)$ and $(R)$-DTBM-SEGPHOS ${ }^{\circledR}$ (64.9 mg, $0.055 \mathrm{mmol}, 5.5 \mathrm{~mol} \%)$ were dissolved in $\mathrm{Et}_{2} \mathrm{O}(3.0 \mathrm{~mL})$ under argon atmosphere. After stirring the solution for $1 \mathrm{~h}$ at $\mathrm{rt}$ the mixture was cooled to $-78{ }^{\circ} \mathrm{C}$ and the cyclopropene substrate $(2,0 \mathrm{~mL}$, $130.2 \mathrm{mg}, 1.0 \mathrm{mmol}, 1.0$ eq.) was added as a $0.5 \mathrm{M}$ solution in pentane.

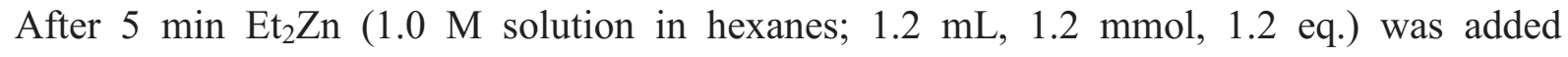
dropwise at the wall of the flask. The reaction was stirred for $3 \mathrm{~h}$ at $-78{ }^{\circ} \mathrm{C}$ then over $3 \mathrm{~h}$ warmed to $0{ }^{\circ} \mathrm{C}$. The reaction mixture was quenched with $\mathrm{HCl}(1.0 \mathrm{M} ; 5.0 \mathrm{~mL})$ and diluted with $\mathrm{Et}_{2} \mathrm{O}(10 \mathrm{~mL})$. The organic phase was separated and the aqueous phase extracted with $\mathrm{Et}_{2} \mathrm{O}(2 \times 10 \mathrm{~mL})$. The combined organic phases were dried over $\mathrm{Na}_{2} \mathrm{SO}_{4}$ and reduced in vacuo. The oily residue was purified by flash chromatography (hexanes) to obtain $\mathbf{3 0}$ as a colorless oil (148 mg, $0.85 \mathrm{mmol}, 85 \%$ yield) and a 4:1 mixture of unassigned diastereomers. $\mathrm{R}_{\mathrm{f}}=0.80$ (Hexanes; stained with phosphomolybdic acid).

${ }^{1} \mathbf{H}$ NMR (400 MHz, $\mathrm{CDCl}_{3}$; mixture of diastereomers) $\delta 7.22-7.06(\mathrm{~m}), 2.62(\mathrm{~d}, J=14.5$ Hz; major), $2.52-2.46(\mathrm{~m}), 2.39$ (d, $J=14.0 \mathrm{~Hz}$, minor), $1.61-1.48(\mathrm{~m}), 1.30-1.16(\mathrm{~m})$, 0.95 (t, $J=7.3 \mathrm{~Hz}$; major), $0.90-0.85$ (m, 1H), 0.84 (s; major), $0.65-0.57$ (m; minor), 0.55 -0.46 (m), 0.39 (dd, $J=8.4,4.2 \mathrm{~Hz}$, major), 0.11 (t, $J=4.8 \mathrm{~Hz}$; major), -0.12 (t, $J=4.8 \mathrm{~Hz}$; minor). ${ }^{13} \mathrm{C}$ NMR (101 MHz, $\mathrm{CDCl}_{3}$; mixture of diastereomers) $\delta 141.3$ (major), 141.0 (minor), 129.38 (minor), 129.35 (major), 128.2 (major), 128.1 (minor), 126.0 (minor), 125.8 (major), 47.4 (minor), 39.9 (major), 27.3 (major), 25.7 (minor), 25.0 (major), 25.0 (minor), 23.4 (major), 23.0 (minor), 20.6 (major), 19.2 (major), 18.6 (minor), 17.5 (minor), 14.6 (major), 14.6 (minor). HRMS (TOF-MS ES ${ }^{+}$): $[\mathrm{M}+\mathrm{H}]^{+}$, calculated for $\mathrm{C}_{13} \mathrm{H}_{19}$ : 175.1487; found 175.1476 .

Note: The enantiomeric ratio of $\mathbf{3 0}$ could not be determined by HPLC or GC with the chiral columns tested (see chemical materials and methods). Therefore, the same enantiomeric ratio as for $\mathbf{3 p}$ was assigned. 


\section{4-((1S,2S,3R)-2-benzyl-3-ethyl-2-methylcyclopropyl)butan-2-one (3p)}

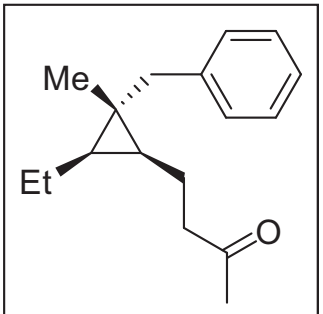

$3 p \mathrm{C}_{17} \mathrm{H}_{24} \mathrm{O}$

MW: 244,37

CuTC (9.5 mg, $0.038 \mathrm{mmol}, 5 \mathrm{~mol} \%)$ and $(R)$-DTBM-SEGPHOS ${ }^{\circledR}$ (48.3 mg, $0.041 \mathrm{mmol}, 5.5 \mathrm{~mol} \%$ ) were dissolved in $\mathrm{Et}_{2} \mathrm{O}(3.0 \mathrm{~mL})$ under argon atmosphere. After stirring the pale pink solution for $1 \mathrm{~h}$ at rt the mixture was cooled to $-78{ }^{\circ} \mathrm{C}$ and the cyclopropene substrate (108.2 $\mathrm{mg}, 0.75 \mathrm{mmol}, 1.0$ eq.) was added as a $0.5 \mathrm{M}$ solution in pentane. After $5 \mathrm{~min}^{\mathrm{Et}}{ }_{2} \mathrm{Zn}(1.0 \mathrm{M}$ solution in hexanes; $0.9 \mathrm{~mL}, 0.9$ mmol, 1.2 eq.) was added dropwise at the wall of the flask over $1 \mathrm{~min}$. After the reaction was stirred for $3 \mathrm{~h}$ at $-78{ }^{\circ} \mathrm{C}$ and $1 \mathrm{~h}$ at $0{ }^{\circ} \mathrm{C}$ the reaction vessel was cooled to $-40{ }^{\circ} \mathrm{C}$ and $\mathrm{CuCN} \cdot 2 \mathrm{LiCl}(0.5 \mathrm{M}$ in THF; $4.0 \mathrm{~mL}, 2.0 \mathrm{mmol}, 2.7$ eq. $)$ was added followed by TMSCl (506 $\mu \mathrm{L}, 430 \mathrm{mg}, 3.96 \mathrm{mmol}, 5.3$ eq.) and methyl vinyl ketone (180 $\mu \mathrm{L}, 151 \mathrm{mg}, 2.16$ mmol, 2.9 eq.). After stirring for $1 \mathrm{~h}$ at $-40{ }^{\circ} \mathrm{C}$ the reaction was slowly warmed over $5 \mathrm{~h}$ to $\mathrm{rt}$ and stirring was continued for $4 \mathrm{~h}$ at rt. The reaction was quenched with $\mathrm{HCl}(1 \mathrm{M}, 5 \mathrm{~mL})$ and stirred for $20 \mathrm{~min}$ at $\mathrm{rt}$. Then $\mathrm{NH}_{4} \mathrm{OH}$ was added until the $\mathrm{pH}$ was $\sim 9$. The reaction mixture was extracted with $\mathrm{Et}_{2} \mathrm{O}(3 \times 10 \mathrm{~mL})$ and the combined ethereal phases washed with dist. $\mathrm{H}_{2} \mathrm{O}$ $(2 \times 10 \mathrm{~mL})$, dried over $\mathrm{Na}_{2} \mathrm{SO}_{4}$ and reduced in vacuo. The oily residue was purified by flash chromatography (hexanes: EtOAc $=20: 1)$ to obtain $\mathbf{3 p}$ as a colorless oil $(124 \mathrm{mg}, 0.51 \mathrm{mmol}$, $68 \%$ yield). $R_{\mathrm{f}}=0.25$ (Hexanes: EtOAc $=20: 1$; stained with anisaldehyde).

${ }^{1} \mathbf{H}$ NMR (400 MHz, $\mathrm{CDCl}_{3}$; mixture of diastereomers) $\delta{ }^{1} \mathrm{H}$ NMR (400 MHz, $\left.\mathrm{CDCl}_{3}\right) \delta 7.23$ - $7.18(\mathrm{~m}), 7.17-7.07(\mathrm{~m}), 2.59$ (d, $J=1.6 \mathrm{~Hz}$; major), 2.46 (t, $J=7.6 \mathrm{~Hz}$; major), 2.37 $2.29(\mathrm{~m}$, minor), 2.09 (s, major), $2.04(\mathrm{~s}$, minor), $1.78-1.66(\mathrm{~m}, 1 \mathrm{H}), 1.60-1.41(\mathrm{~m}, 1 \mathrm{H})$, $1.34-1.16(\mathrm{~m}, 1 \mathrm{H}), 0.95$ (t, $J=7.3 \mathrm{~Hz}$; major), 0.87 (s; major), $0.86-0.83$ (m, minor), 0.76 (s, minor), $0.63-0.58$ (m, minor), $0.53-0.46$ (m, major). ${ }^{13} \mathbf{C}$ NMR (101 MHz, $\mathrm{CDCl}_{3}$; mixture of diastereomers) $\delta 209.3$ (minor), 209.2 (major), 140.9 (minor), 140.5 (major), 129.5 (major), 129.3 (minor), 128.2 (major), 128.1 (minor), 126.0 (minor), 125.8 (major), 49.0 (minor), 44.6 (major), 44.2 (minor), 35.2 (major), 30.2 (major), 30.1 (minor), 29.4 (major), 27.8 (minor), 27.1 (major), 26.6 (major), 24.8 (minor), 22.6 (minor), 21.6 (major), 19.4 (major), 18.9 (minor), 18.1 (major), 17.6 (minor), 15.0 (major), 14.6 (minor), 12.2 (minor).

HRMS (TOF-MS ES ${ }^{+}$):[M+H] $]^{+}$, calculated for $\mathrm{C}_{17} \mathrm{H}_{25} \mathrm{O}$ : 245.1900; found 245.1896. HPLC analysis analysis (CHIRALCEL OD, 1\% IPA: 99\% hexanes, $1.0 \mathrm{~mL} / \mathrm{min}, 254 \mathrm{~nm}, \mathrm{t}_{\mathrm{R} 1}=7.63$ $\min , t_{R 2}=8.93 \mathrm{~min}$ ) indicated 99.3:0.7 er. Note: First peak in HPLC trace belongs to the minor diastereomer. We were unable to find conditions to efficiently separate the enantiomers of the minor diastereomer. 


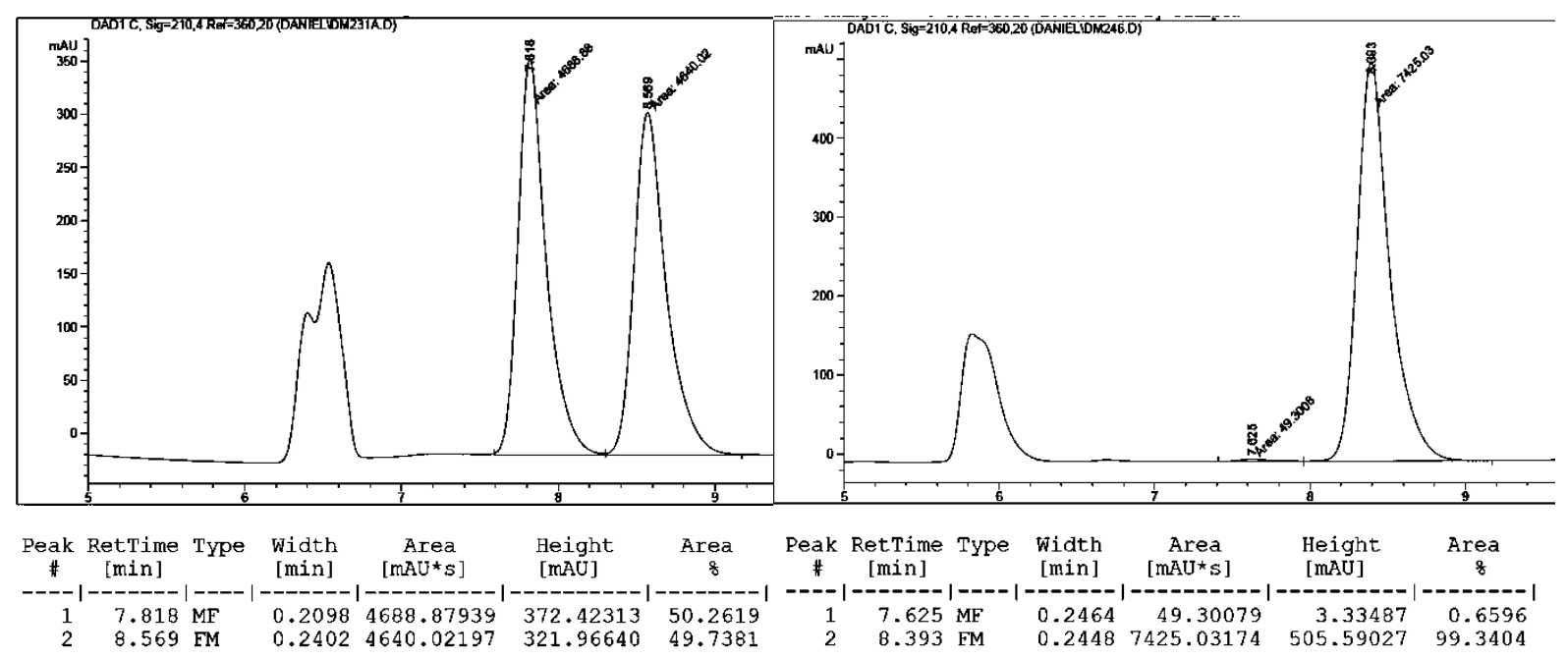

\section{4-((1S,3R)-3-ethyl-2,2-diphenylcyclopropyl)butan-2-one (3q)}

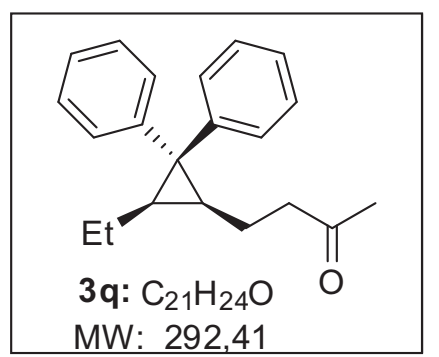

Ketone $\mathbf{3 q}$ was prepared according to general procedure GP2 $(0.5$ mmol scale). The catalyst loading was $5.0 \mathrm{~mol} \%$ (optimized). A prolonged reaction time of $8 \mathrm{~h}$ at $0{ }^{\circ} \mathrm{C}$ was necessary to achieve full conversion for the carbozincation. After standard work-up the oily residue was purified by flash chromatography (hexanes: EtOAc $=20: 1$ to $10: 1)$ to obtain $\mathbf{3 q}$ as a colorless viscous oil $(68$ mg, 0.23 mmol, 47\% yield). $R_{\mathrm{f}}=0.32$ (Hexanes: EtOAc $=10: 1$; stained with anisaldehyde).

${ }^{1} \mathbf{H}$ NMR $\left(400 \mathrm{MHz}, \mathrm{CDCl}_{3}\right) \delta 7.28$ - $7.22(\mathrm{~m}, 4 \mathrm{H}), 7.19-7.09(\mathrm{~m}, 3 \mathrm{H}), 7.08-6.98(\mathrm{~m}, 3 \mathrm{H})$, $2.67-2.51(\mathrm{~m}, 2 \mathrm{H}), 2.09(\mathrm{~s}, 3 \mathrm{H}), 1.94-1.81(\mathrm{~m}, 1 \mathrm{H}), 1.64-1.52(\mathrm{~m}, 1 \mathrm{H}), 1.45-1.25(\mathrm{~m}$, $3 \mathrm{H}), 1.22-1.11(\mathrm{~m}, 1 \mathrm{H}), 1.08(\mathrm{t}, J=6.5 \mathrm{~Hz}, 3 \mathrm{H}) .{ }^{13} \mathbf{C} \mathbf{N M R}\left(101 \mathrm{MHz}, \mathrm{CDCl}_{3}\right) \delta 208.6$, $149.1,139.5,131.7,128.6,128.3,127.2,126.5,125.5,44.2,37.5,32.0,30.1,29.3,20.9$, 19.7, 14.9. HRMS TOF-MS ES+: $[\mathrm{M}+\mathrm{H}]^{+}$, calculated for $\mathrm{C}_{21} \mathrm{H}_{25} \mathrm{O}$ : 293.1900; found 293.1898 . HPLC analysis (CHIRALCEL OD, 0.5\% IPA: 99.5\% hexanes, $1.0 \mathrm{~mL} / \mathrm{min}, 254 \mathrm{~nm}, \mathrm{t}_{\mathrm{R} 1}=$ $\left.12.3 \mathrm{~min}, \mathrm{t}_{\mathrm{R} 2}=15.3 \mathrm{~min}\right)$ indicated $90: 10 \mathrm{er} .[\alpha]_{\mathbf{D}}{ }^{20}=-6.6\left(\mathrm{c}=0.36, \mathrm{CHCl}_{3}\right)$. 

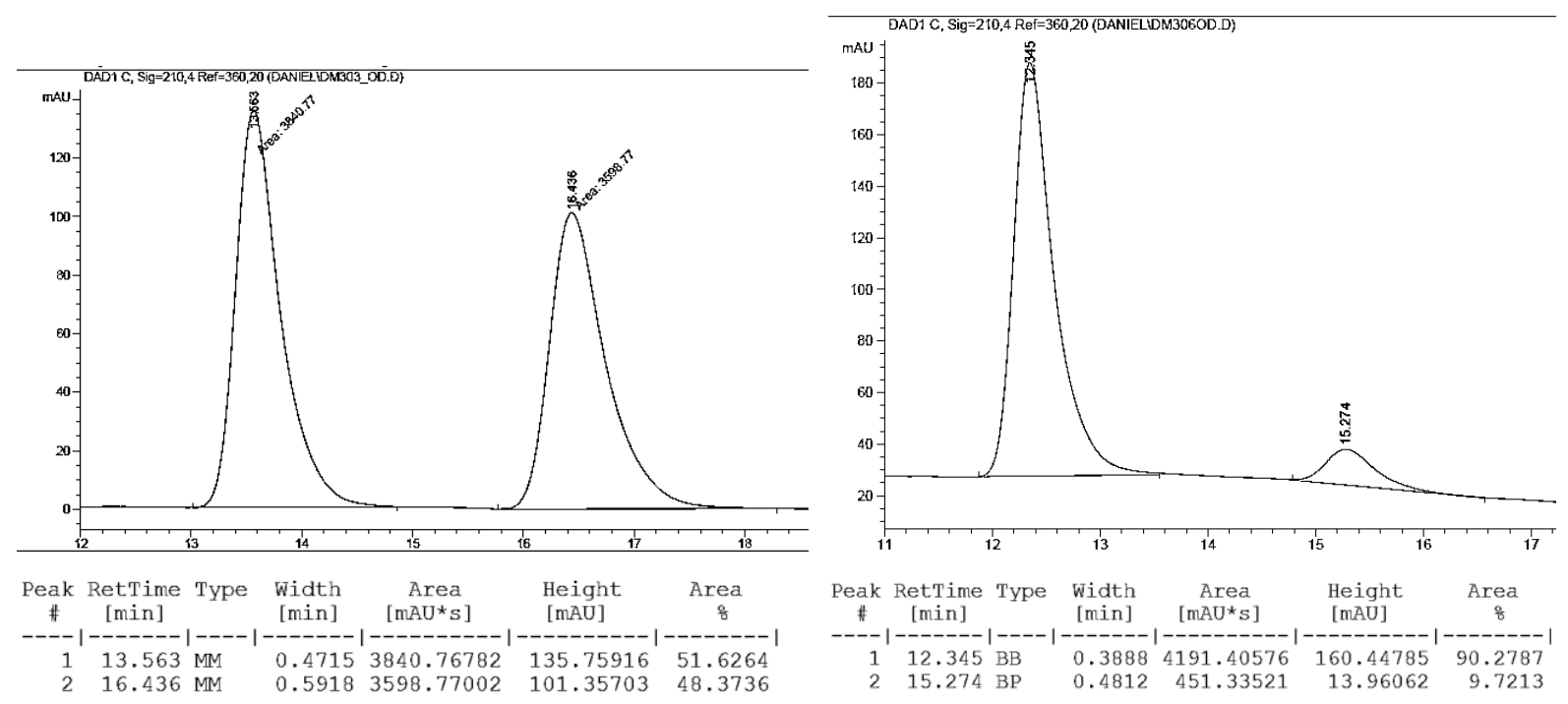

\section{1-(4-((1R,2S,3R)-2-tert-butyl-3-ethyl-2-methylcyclopropyl)phenyl)ethanone (3r)}

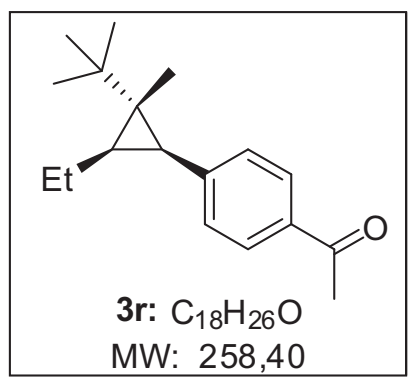

CuTC (11.4 mg, $0.06 \mathrm{mmol}, 10.0 \mathrm{~mol} \%)$ and $(R)$-DTBMSEGPHOS $^{\circledR}(77.9 \mathrm{mg}, 0.066 \mathrm{mmol}, 11.0 \mathrm{~mol} \%)$ were dissolved in $\mathrm{Et}_{2} \mathrm{O}(2.5 \mathrm{~mL})$ under argon atmosphere. After stirring the solution for $1 \mathrm{~h}$ at $\mathrm{rt}$ the mixture was cooled to $-78^{\circ} \mathrm{C}$ and cyclopropene $1 \mathrm{~h}$ (1.0 mL, $55.1 \mathrm{mg}, 0.6 \mathrm{mmol}, 1.0 \mathrm{eq} ; 0.6 \mathrm{M}$ solution in pentane) was added. After 5 min $\mathrm{Et}_{2} \mathrm{Zn}(1.0 \mathrm{M}$ solution in hexanes; 0.72

$\mathrm{mL}, 1.2 \mathrm{mmol}, 1.2$ eq.) was added dropwise at the wall of the flask. The reaction was stirred for $3 \mathrm{~h}$ at $-78{ }^{\circ} \mathrm{C}$ then was warmed to $0{ }^{\circ} \mathrm{C}$ over $3 \mathrm{~h}$ (GC-analysis showed $30 \%$ conversion after $3 \mathrm{~h}$ at $-78{ }^{\circ} \mathrm{C}$ ). To the in situ generated cyclopropylzinc was added a solution of $\mathrm{Pd}(\mathrm{OAc})_{2}(5.4 \mathrm{mg}, 0.024 \mathrm{mmol}, 0.04$ eq.) and SPhos (32.9 mg, $0.048 \mathrm{mmol}, 0.08$ eq.) in THF $(1.0 \mathrm{~mL})$ followed by 4-bromoacetphenone (298 mg, $1.5 \mathrm{mmol}, 2.5$ eq.). Stirring at $\mathrm{rt}$ was continued for $16 \mathrm{~h}$. Then the reaction mixture was treated with $\mathrm{HCl}(1 \mathrm{M}, 5 \mathrm{~mL})$ and extracted with $\mathrm{Et}_{2} \mathrm{O}(3 \times 15 \mathrm{~mL})$. The combined organic phases were dried over $\mathrm{Na}_{2} \mathrm{SO}_{4}$ and reduced in vacuo. The oily residue purified by flash chromatography (hexanes / ethyl acetate $=50: 1$ to $30: 1)$ to obtain $3 \mathbf{r}$ as a pale yellow oil (124 $\mathrm{mg}, 0.48 \mathrm{mmol}, 80 \%$ yield). $\mathrm{R}_{\mathrm{f}}=0.45$ (Hexanes: EtOAc $=10: 1 ;$ stained with phosphomolybdic acid).

${ }^{1} \mathbf{H}$ NMR (400 MHz, $\left.\mathrm{CDCl}_{3}\right) \delta 7.85(\mathrm{appd}, J=8.3 \mathrm{~Hz}, 2 \mathrm{H}), 7.22(\operatorname{appd}, J=7.8 \mathrm{~Hz}, 2 \mathrm{H}), 2.56$ (s, 3H), $2.04(\mathrm{~d}, J=9.2 \mathrm{~Hz}, 1 \mathrm{H}), 1.70-1.59(\mathrm{~m}, 1 \mathrm{H}), 1.19-1.12(\mathrm{~m}, 1 \mathrm{H}), 1.11-1.01$ (m, 1H), $1.00-0.96$ (m, 3H), 0.95 (s, 9H), 0.77 (s, 3H). $\left.{ }^{13} \mathbf{C ~ N M R ~ ( 1 0 1 ~ M H z , ~} \mathrm{CDCl}_{3}\right) \delta$ 198.0, 145.6, 134.7, 131.3, 128.2, 33.0, 30.6, 27.1, 26.9, 26.6, 25.9, 19.6, 14.8, 11.8. HRMS (APCI): $[\mathrm{M}+\mathrm{H}]^{+}$, calculated for $\mathrm{C}_{18} \mathrm{H}_{27} \mathrm{O}: 259.2056$; found 259.2063. HPLC analysis (CHIRALCEL 
OD, $0.5 \%$ IPA: $99.5 \%$ hexanes, $1.0 \mathrm{~mL} / \mathrm{min}, 254 \mathrm{~nm}, \mathrm{t}_{\mathrm{R} 1}=5.9 \mathrm{~min}, \mathrm{t}_{\mathrm{R} 2}=6.2 \mathrm{~min}$ ) indicated $96: 4$ er. $[\alpha]_{\mathbf{D}}{ }^{20}=+30.7\left(\mathrm{c}=0.76 . \mathrm{CHCl}_{3}\right)$.
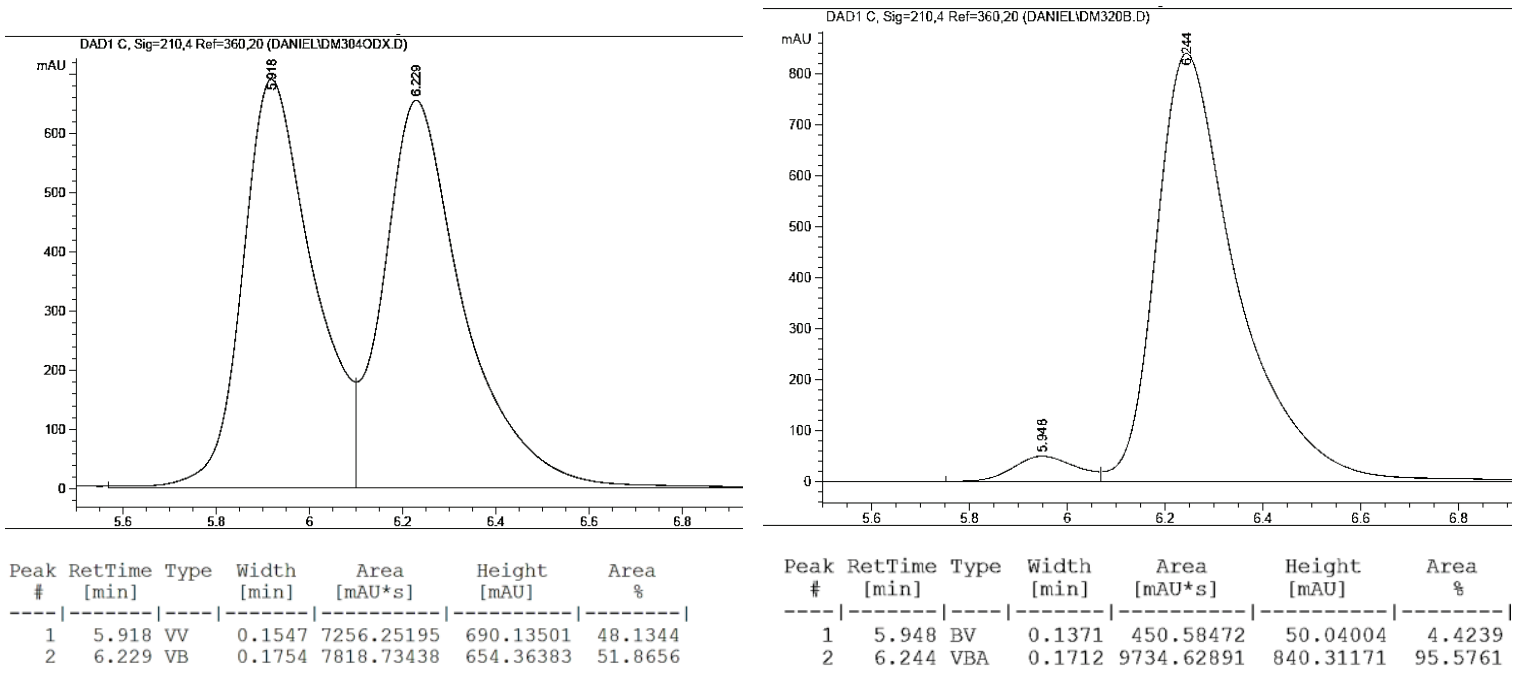

\section{$5 \quad$ References}

[1] T. Nakanoa, K. Endo, Y. Ukajia, Synlett 2015, 26, 671-675.

[2] D. Müller, A. Alexakis, Chem. Eur. J. 2013, 19, 15226-15239.

[3] A. Berkessel, P. Kaiser, J. Lex, Chem. Eur. J. 2003, 9, 4746-4756.

[4] A. Parra, L. Amenós, M. Guisán-Ceinos, A. López, J. L. Ruano, M. Tortosa, J. Am. Chem. Soc. 2014, 136, 15833-15836. 


\section{$6 \quad$ NMR Spectra}

D:/Daniel/Technion/Lab/NMR/NMR-Data/ 19

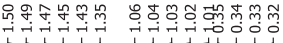
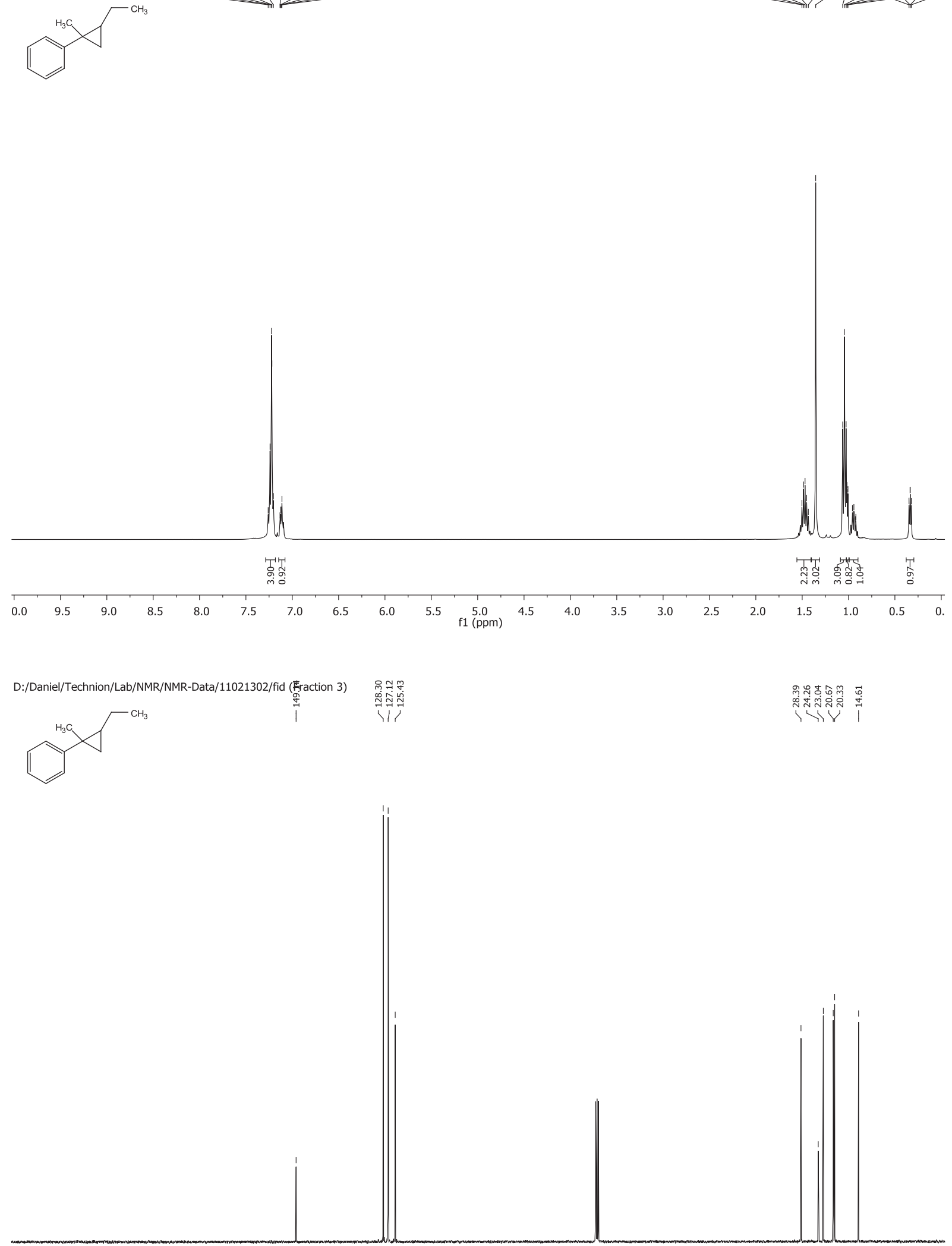

$\begin{array}{llllllllllll}210 & 200 & 190 & 180 & 170 & 160 & 150 & 140 & 130 & 120 & 110 & 100\end{array}$ 


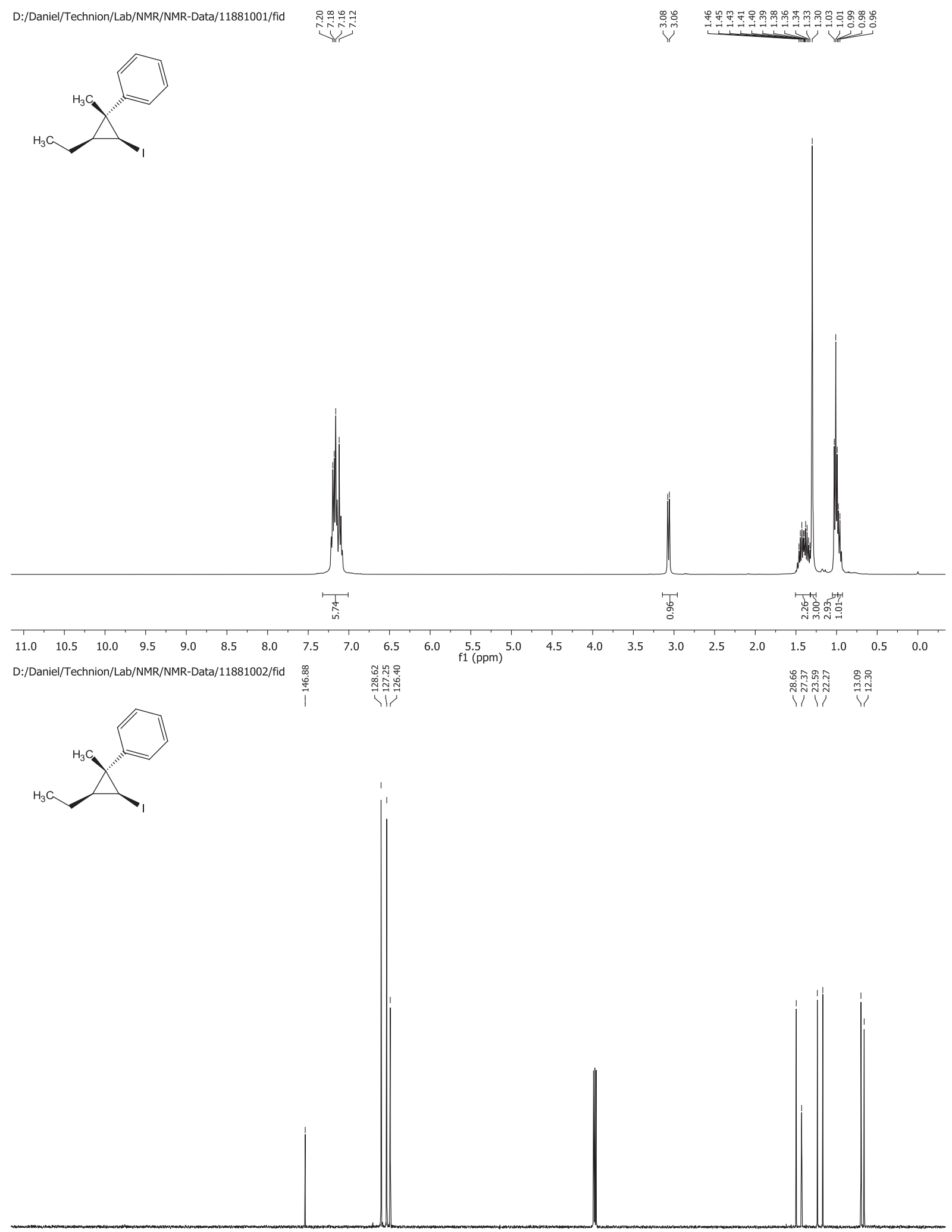

$\begin{array}{lllllllllllllllllllllll}210 & 200 & 190 & 180 & 170 & 160 & 150 & 140 & 130 & 120 & 110 & 100 & 90 & 80 & 70 & 60 & 50 & 40 & 30 & 20 & 10 & 0\end{array}$ 

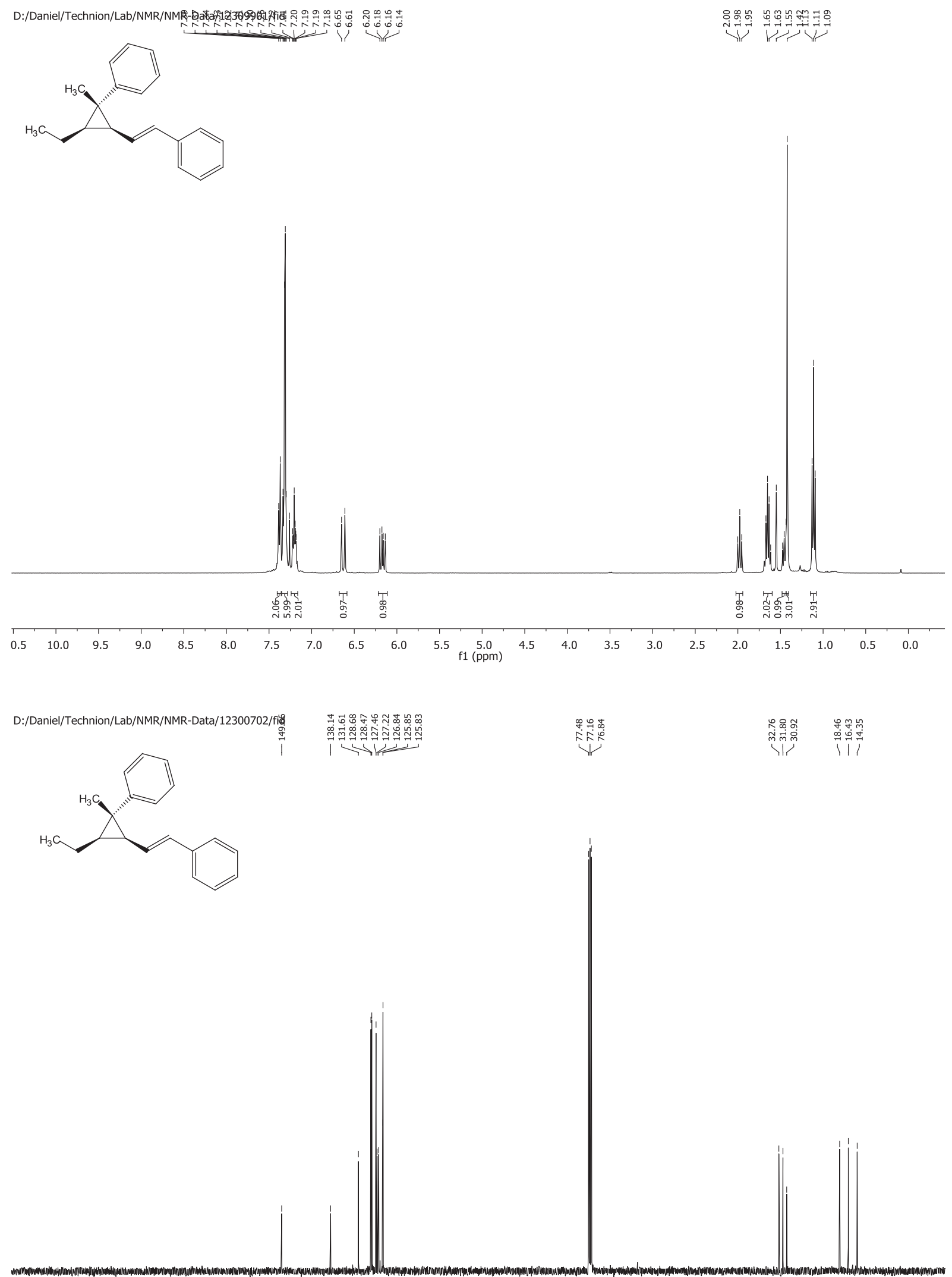

$\begin{array}{lllllllllllllllllllllllll}210 & 200 & 190 & 180 & 170 & 160 & 150 & 140 & 130 & 120 & 110 & 100 & 90 & 80 & 70 & 60 & 50 & 40 & 30 & 20 & 10 & 0\end{array}$ 


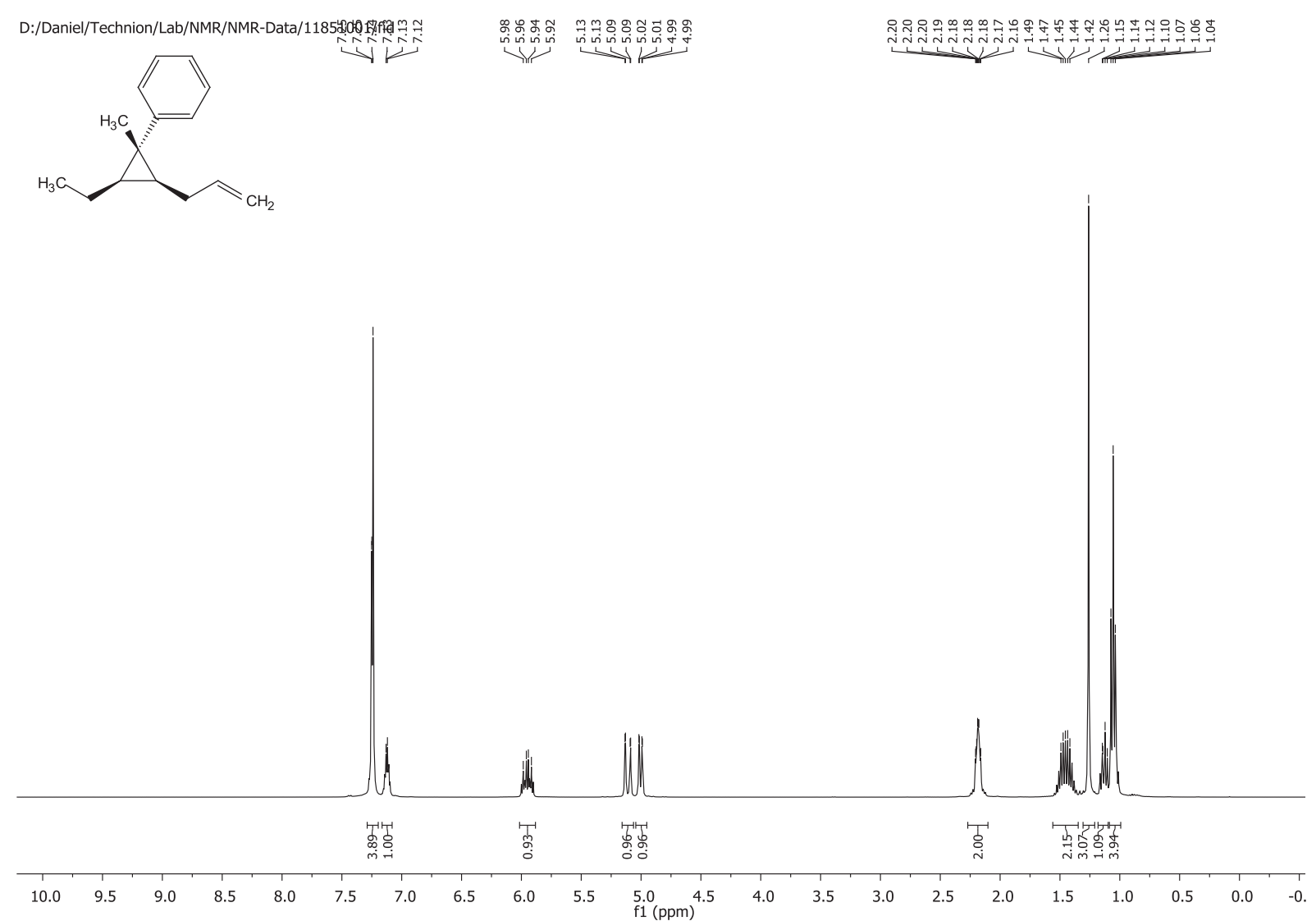

D:/Daniel/Technion/Lab/NMR/NMR-Data/11851004/f梅

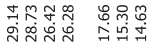
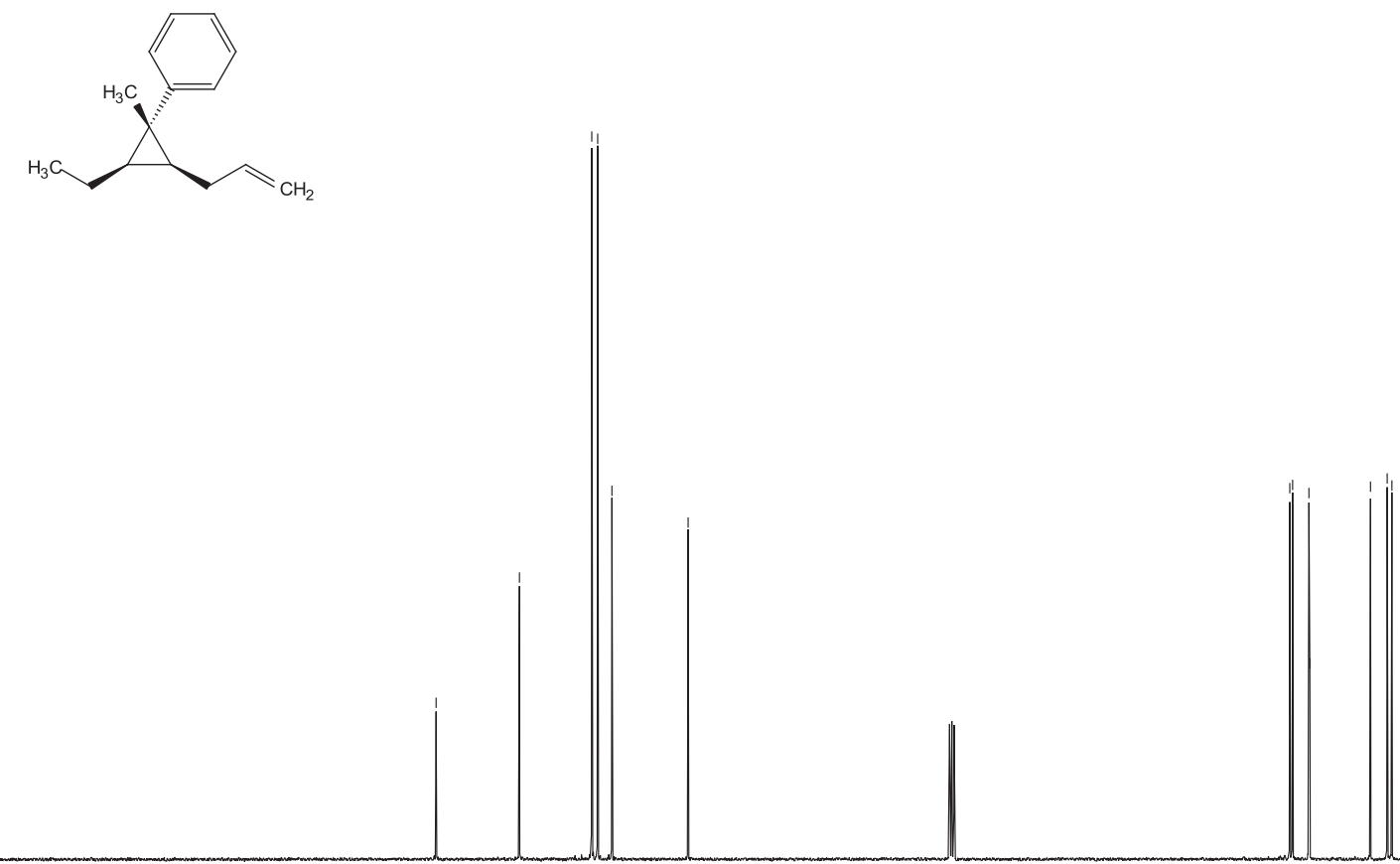

$\begin{array}{lllllllllll}210 & 200 & 190 & 180 & 170 & 160 & 150 & 140 & 130 & 120 & 110 \begin{array}{r}100 \\ \mathrm{f} 1(\mathrm{ppm})\end{array}\end{array}$ 

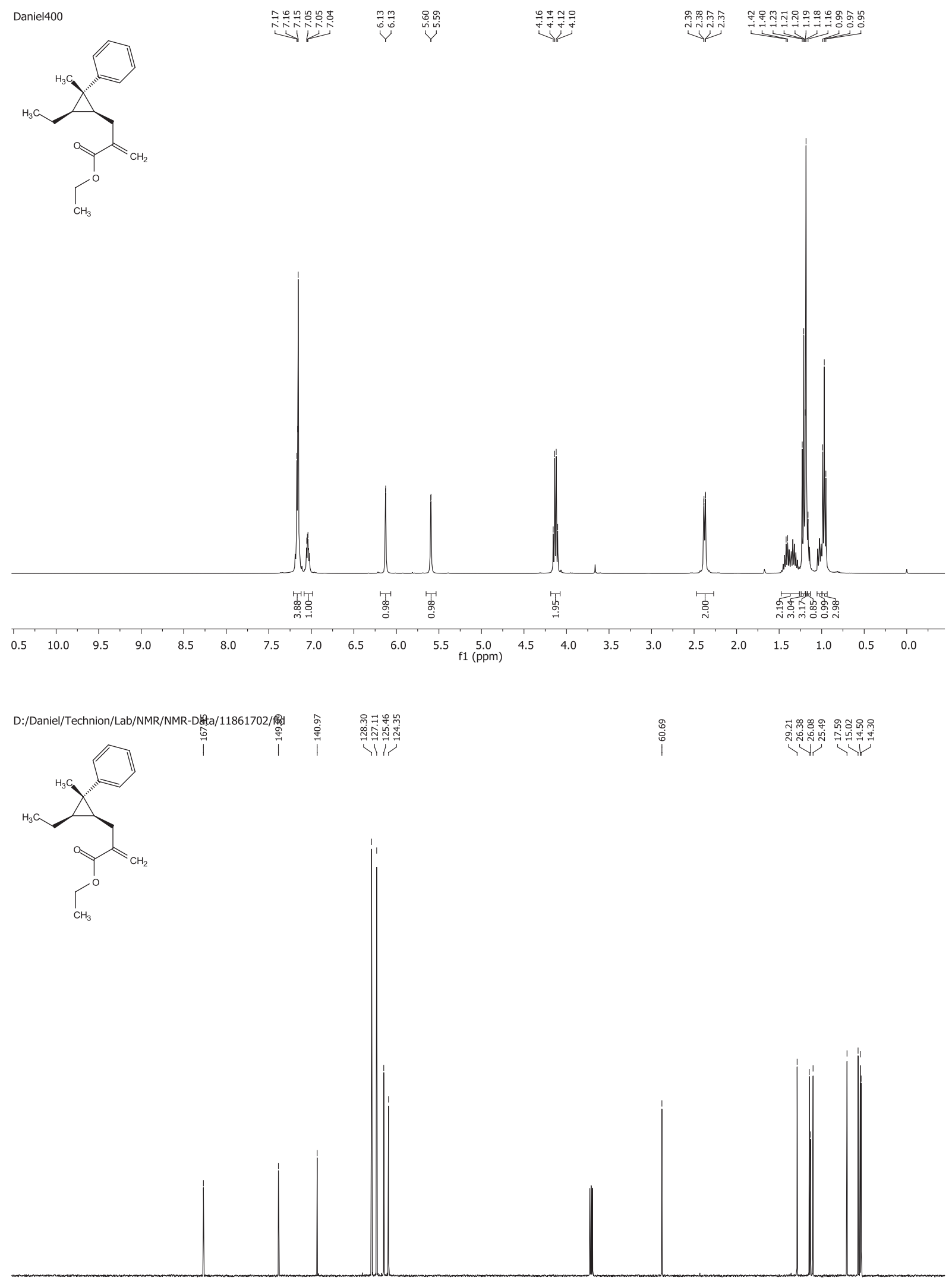

\begin{tabular}{llllllllllllllllllllllll}
\hline & 210 & 200 & 190 & 180 & 170 & 160 & 150 & 140 & 130 & 120 & 110 & 100 & 90 & 80 & 70 & 60 & 50 & 40 & 30 & 20 & 10 & 0
\end{tabular} 

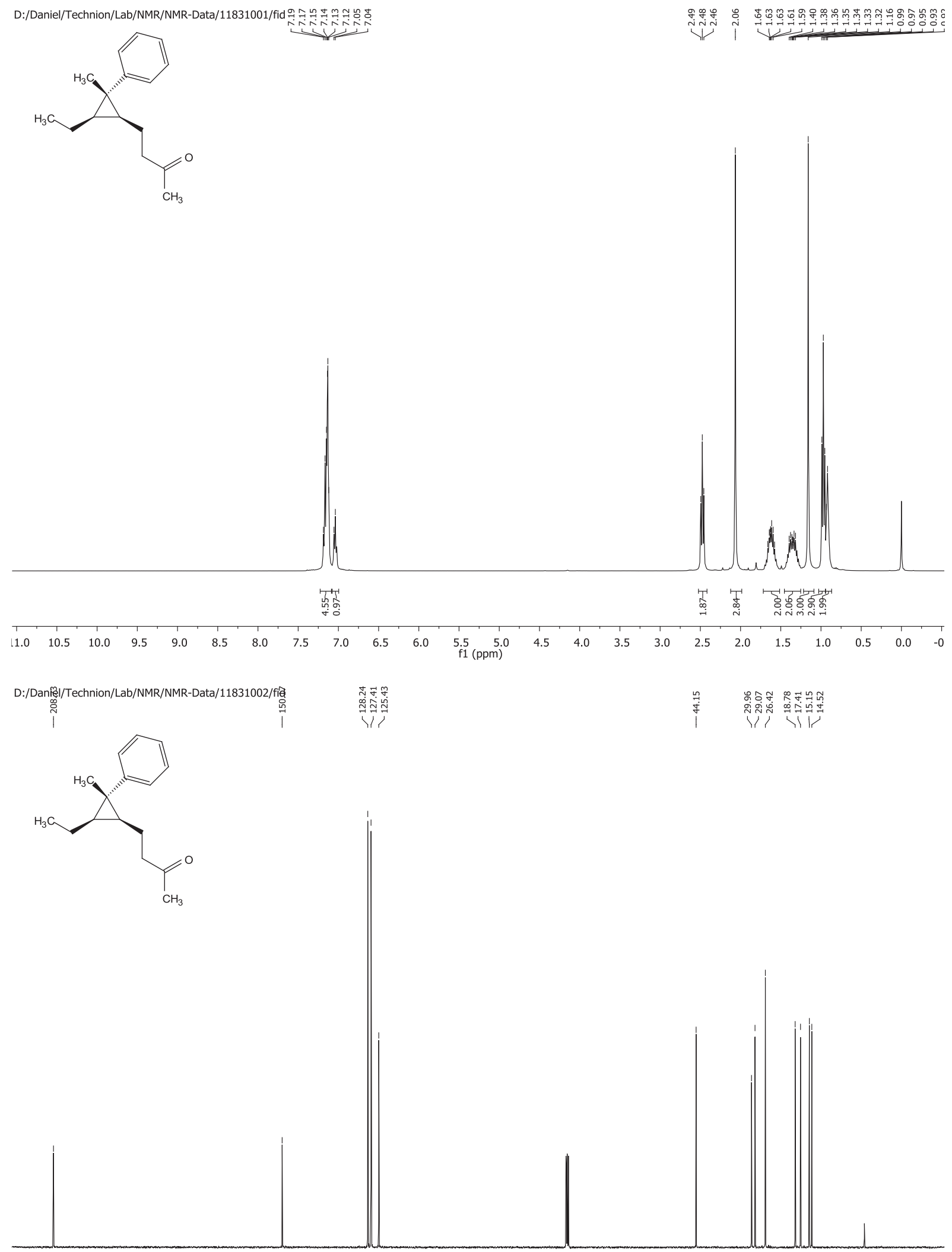

$\begin{array}{lllllllllllllllllllllllll}210 & 200 & 190 & 180 & 170 & 160 & 150 & 140 & 130 & 120 & 110 & 100 & 90 & 80 & 70 & 60 & 50 & 40 & 30 & 20 & 10 & 0 & -10\end{array}$ 
D:/Daniel/Technion/Lab/NMR/NMR-Data/1239090aj/Afaf

$\underbrace{\vec{n}}$
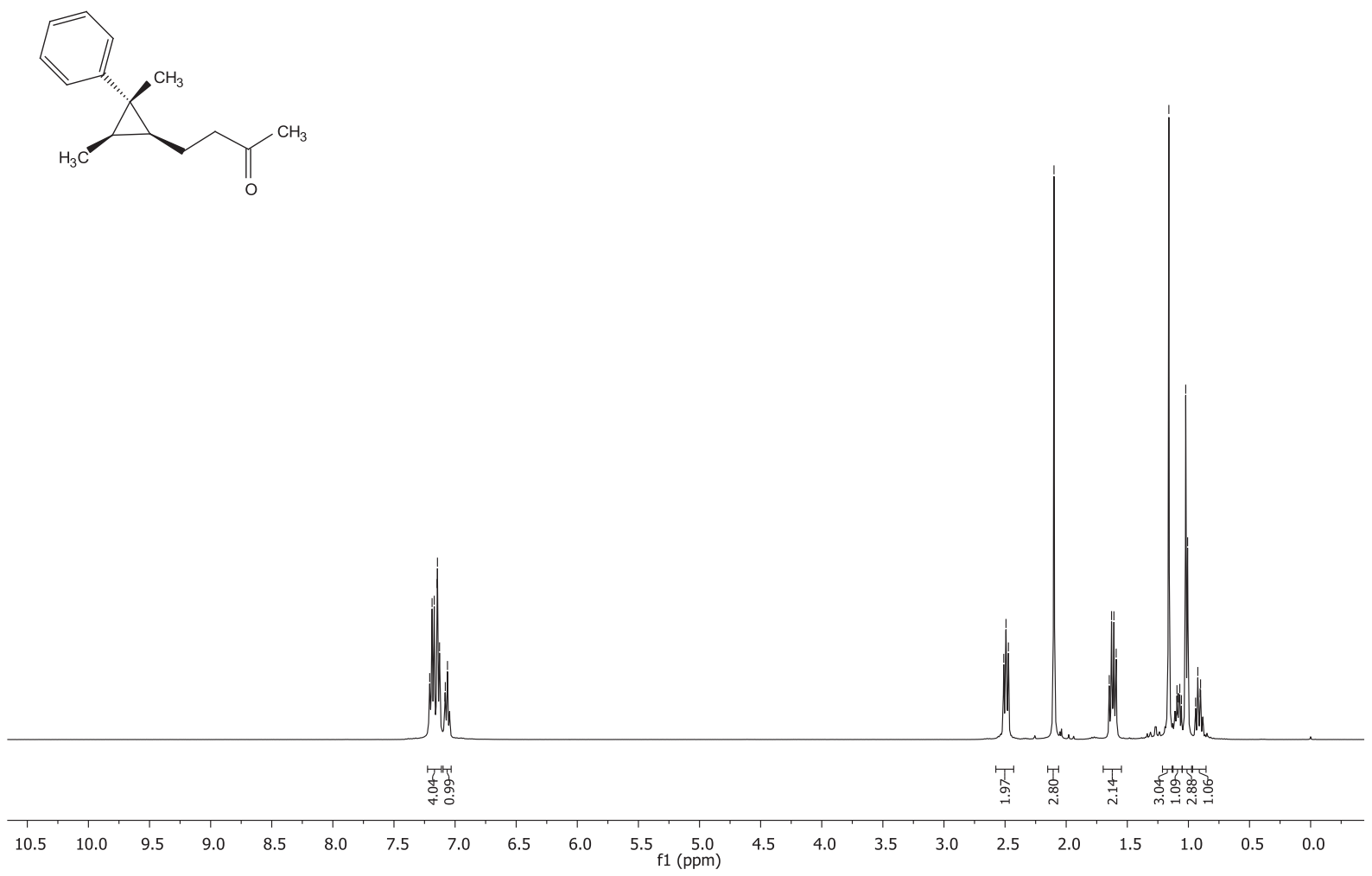

Daniel4\%

Daniel4

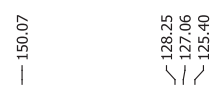

|
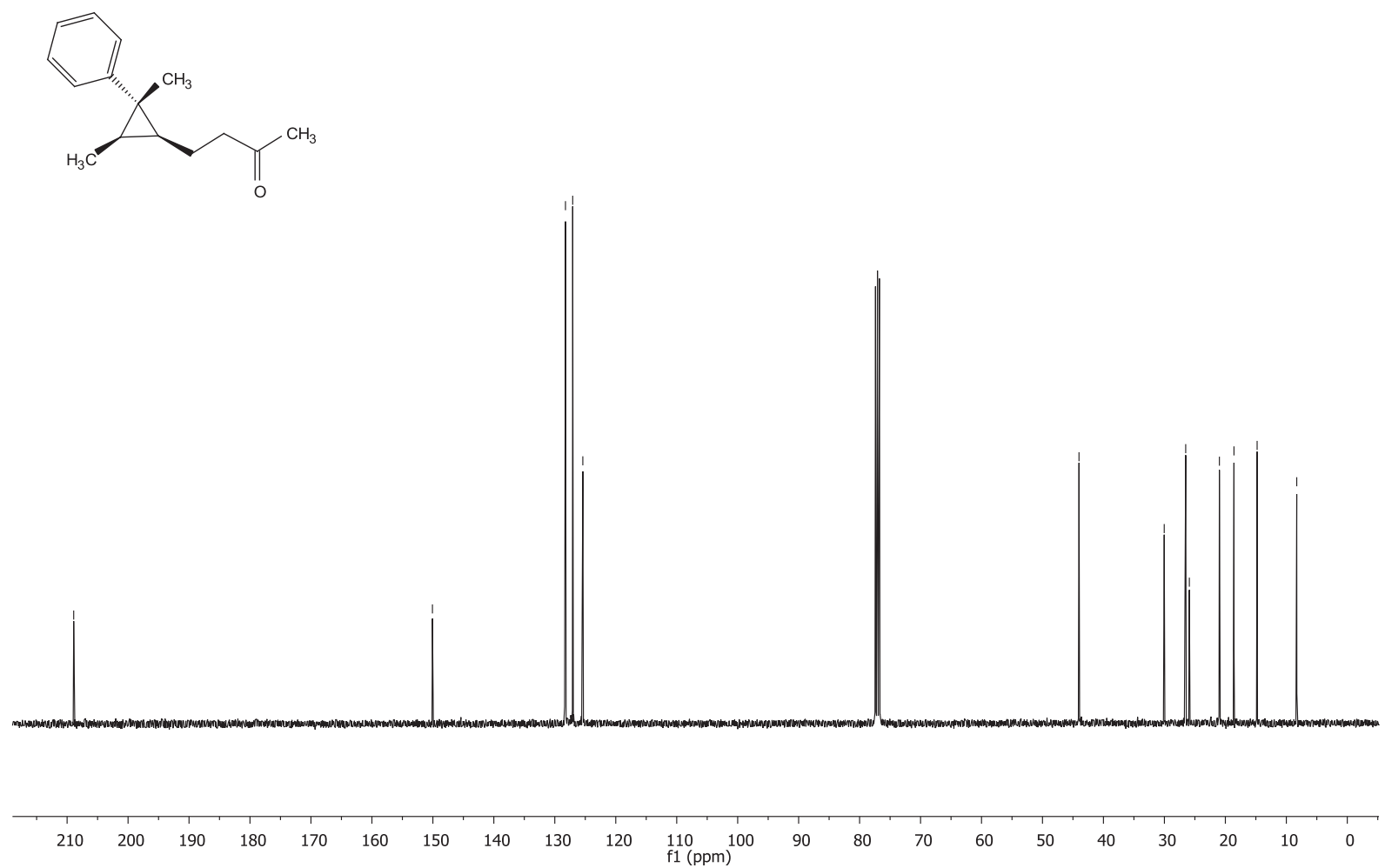

S31 

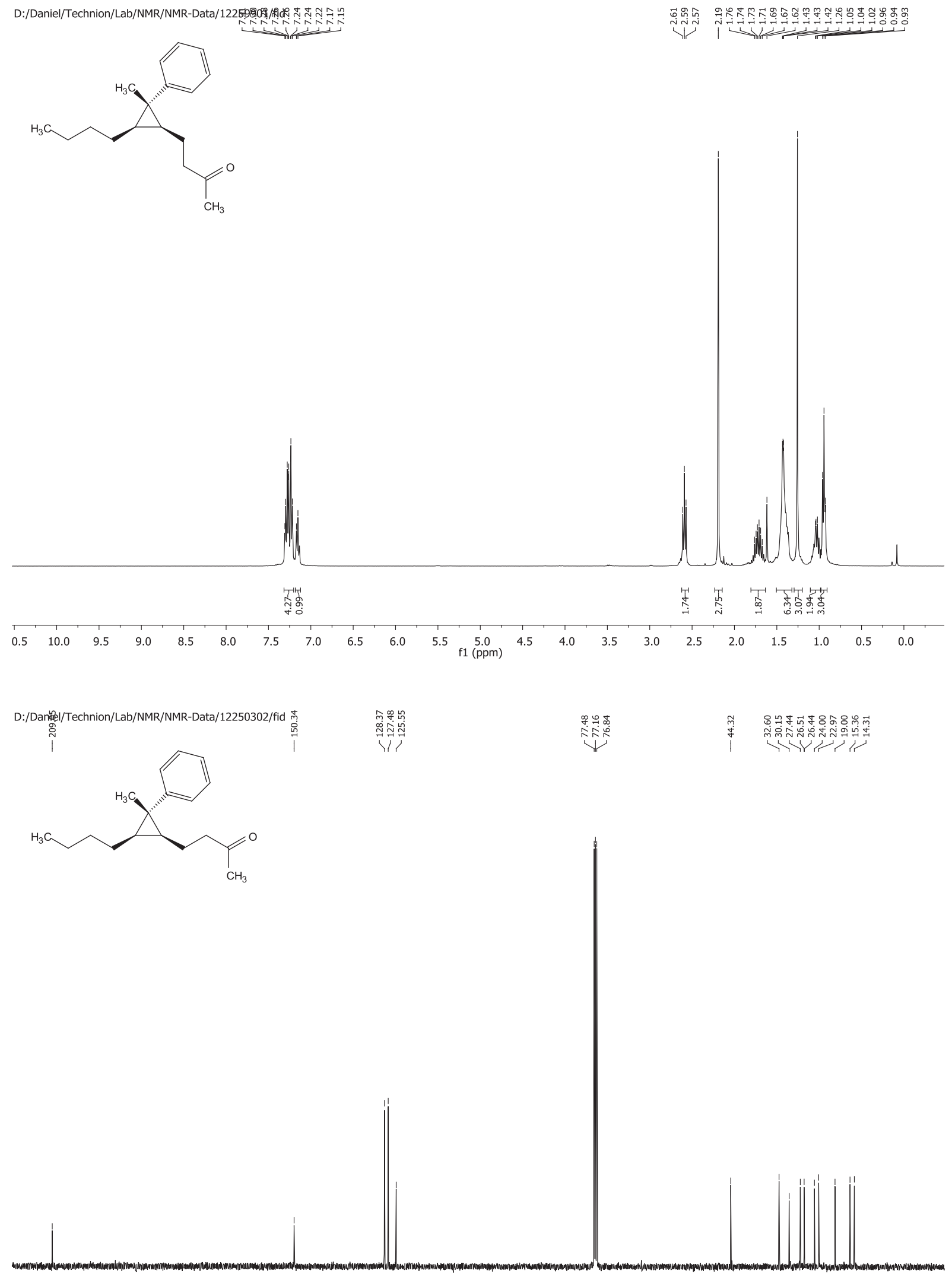

$\begin{array}{lllllllllllllllllllllllllll}210 & 200 & 190 & 180 & 170 & 160 & 150 & 140 & 130 & 120 & 110 & 100 & 90 & 80 & 70 & 60 & 50 & 40 & 30 & 20 & 10 & 0\end{array}$ 

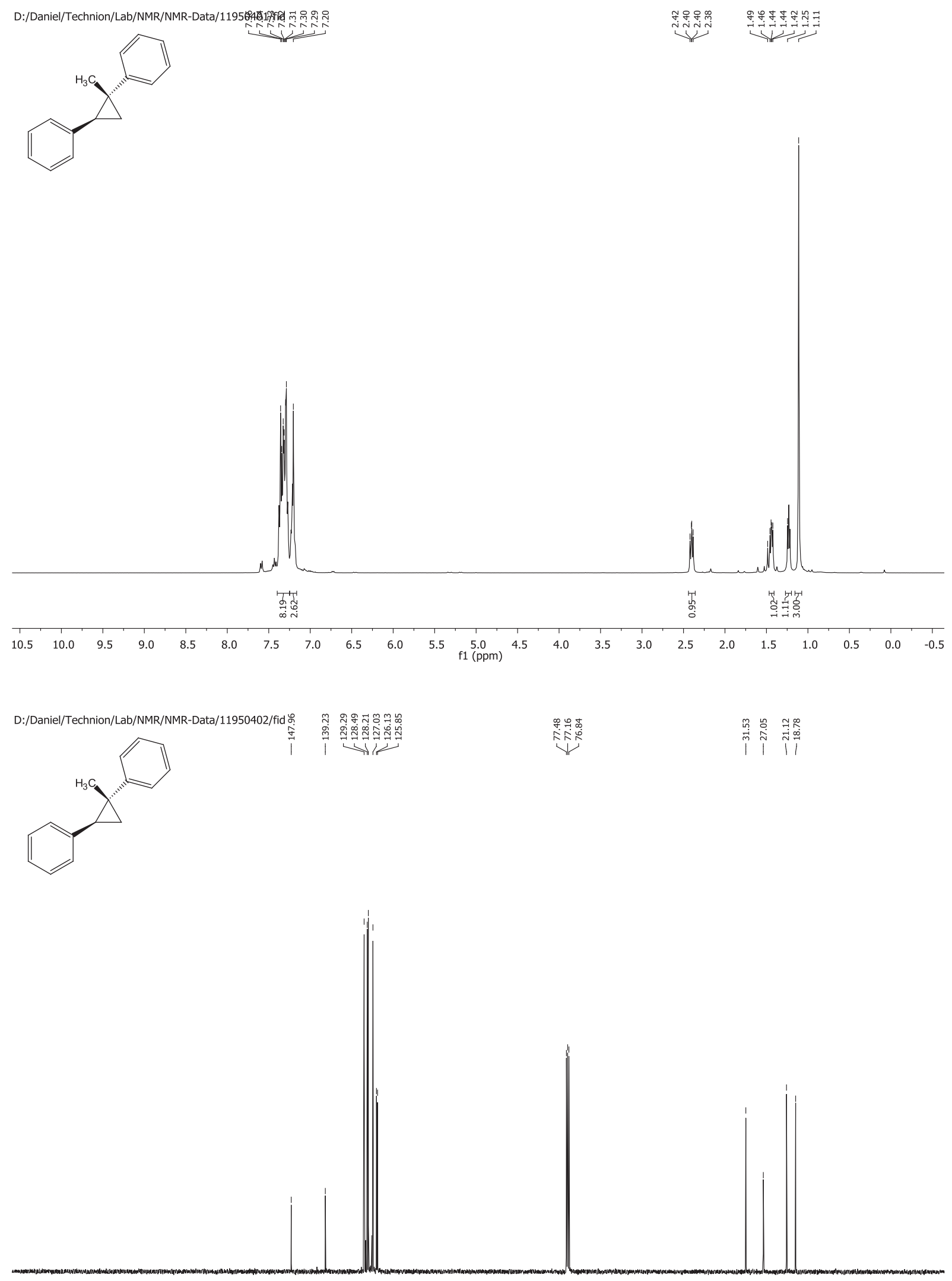

$\begin{array}{llllllllllllllllllllllllllll}210 & 200 & 190 & 180 & 170 & 160 & 150 & 140 & 130 & 120 & 110 & 100 & 90 & 80 & 70 & 60 & 50 & 40 & 30 & 20 & 10 & 0 & -10\end{array}$ 

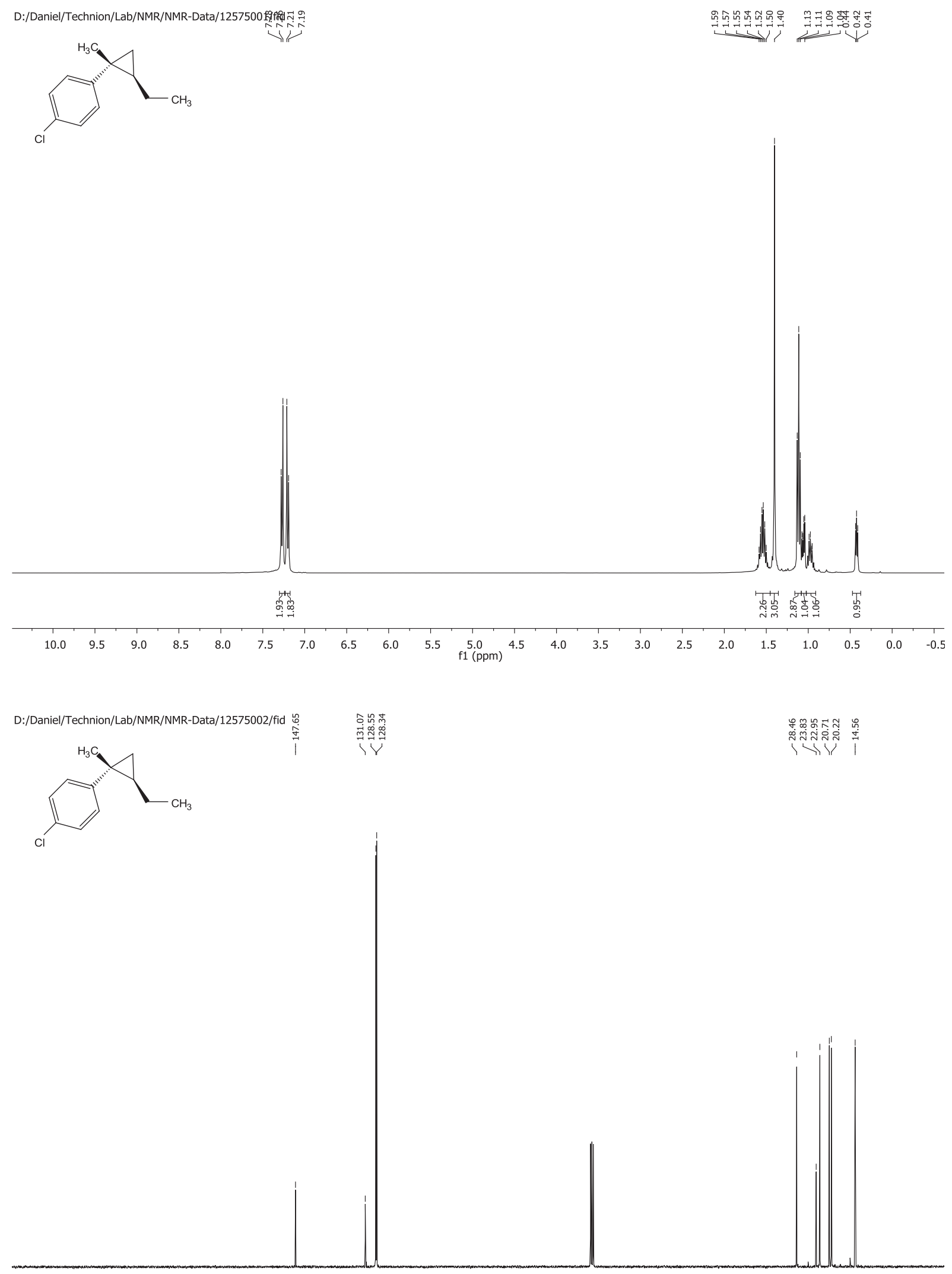

$\begin{array}{lllllllllllllllllllll}210 & 200 & 190 & 180 & 170 & 160 & 150 & 140 & 130 & 120 & \underset{\mathrm{f} 1(\mathrm{ppm})}{110} & 90 & 80 & 70 & 60 & 50 & 40 & 30 & 20 & 10 & 0\end{array}$ 

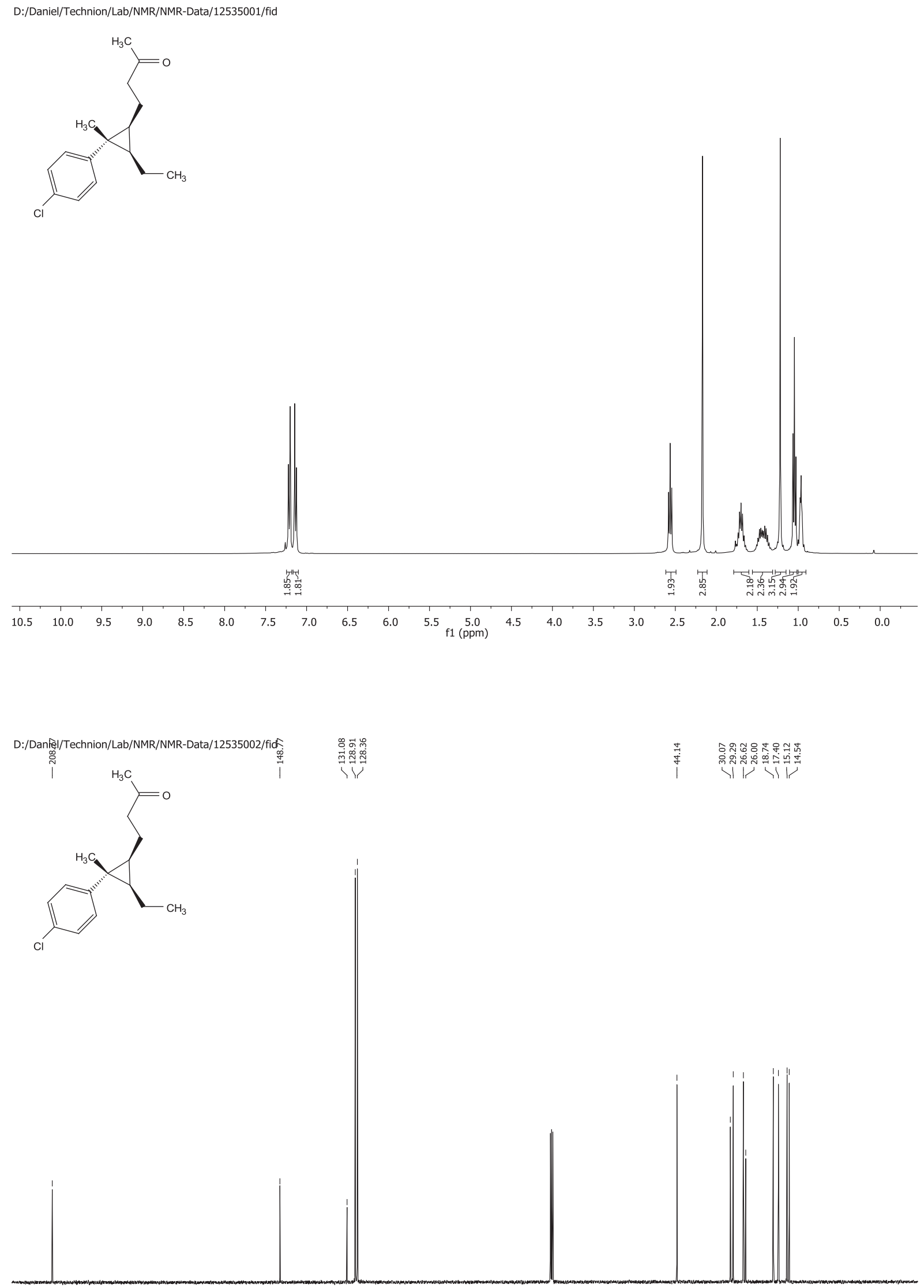

$\begin{array}{lllllllllllllllllllllll}210 & 200 & 190 & 180 & 170 & 160 & 150 & 140 & 130 & 120 & 110 & \begin{array}{c}100 \\ \mathrm{f} 1(\mathrm{ppm})\end{array} & 90 & 80 & 70 & 60 & 50 & 40 & 30 & 20 & 10 & 0 & -10\end{array}$ 


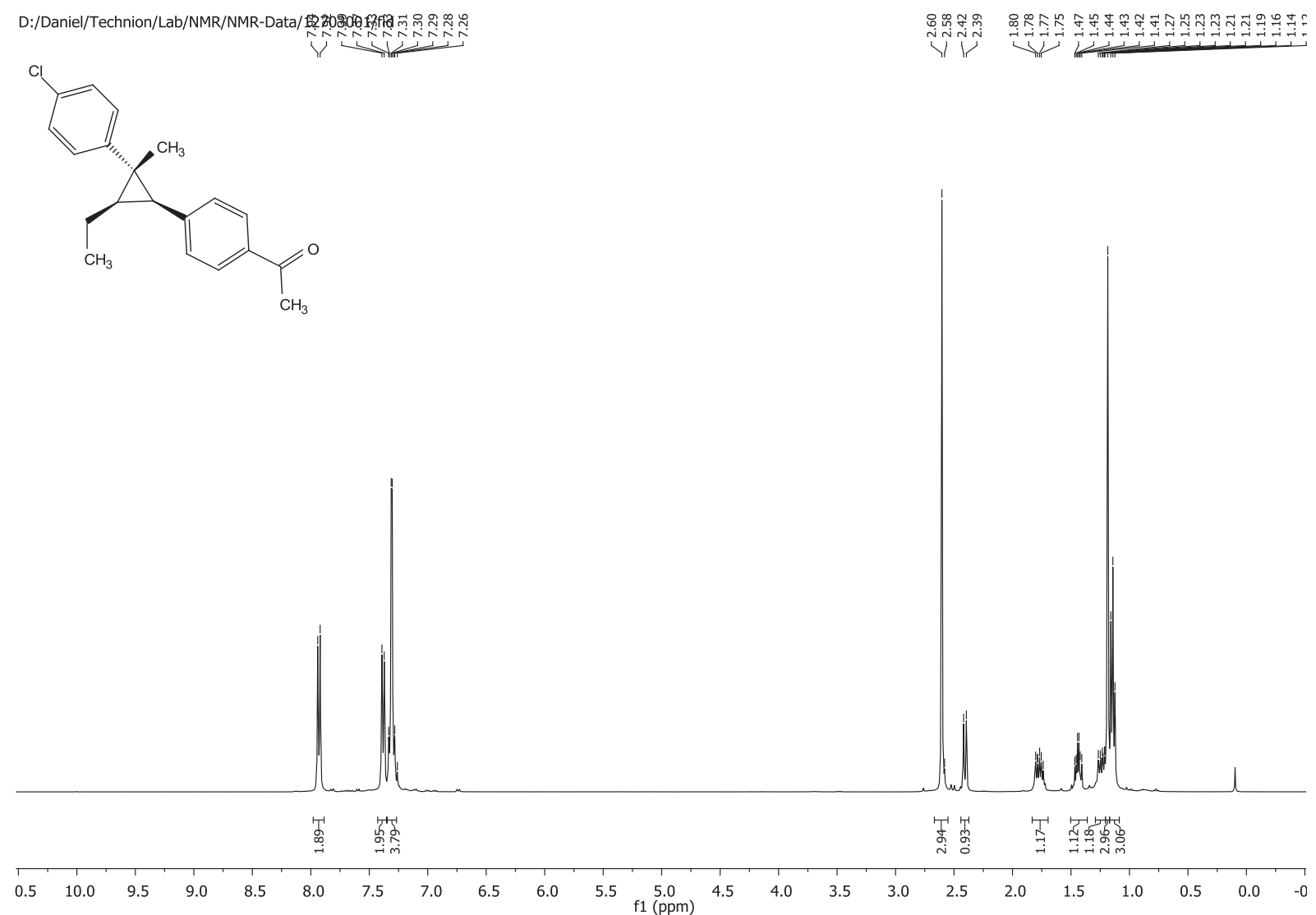

D:/Daniel/Techägon/Lab/NMR/NMR-Data/12703002/fid

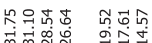

लिख्र
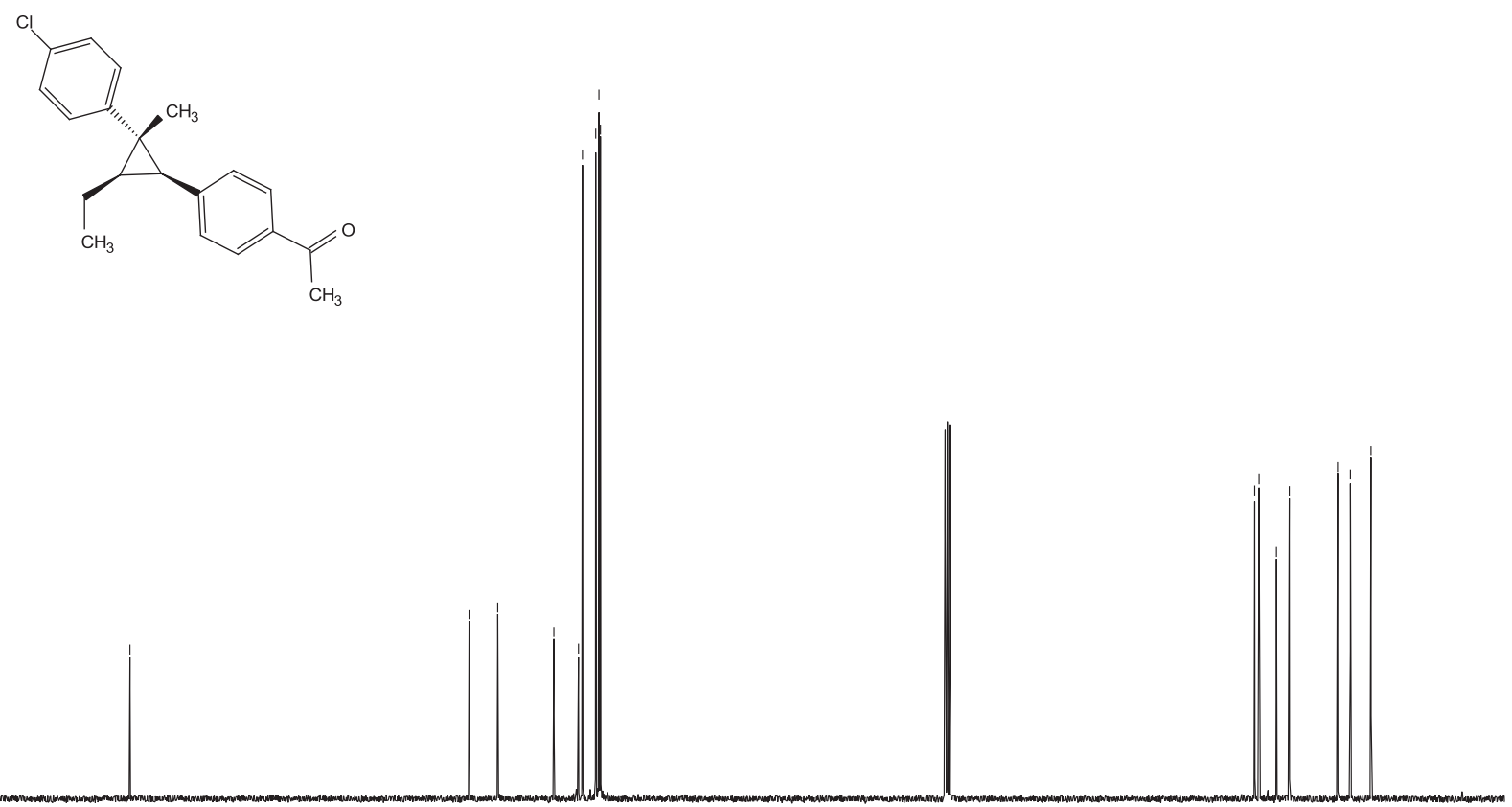

$\begin{array}{lllllllllllllllllllllllll}210 & 200 & 190 & 180 & 170 & 160 & 150 & 140 & 130 & 120 & 110 & 100 & 90 & 80 & 70 & 60 & 50 & 40 & 30 & 20 & 10 & 0\end{array}$ 


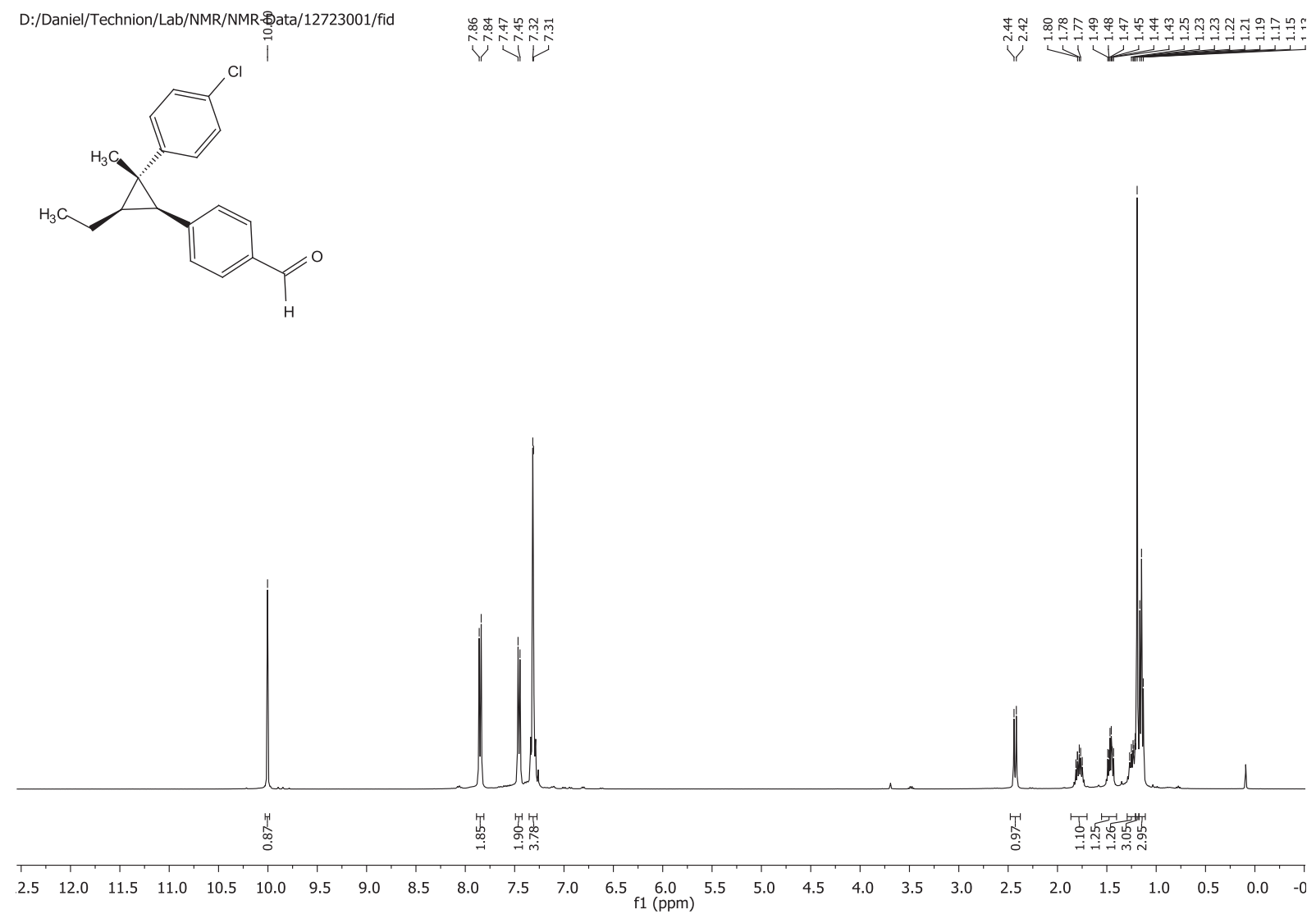<smiles>CCC1C(c2ccc(C(=O)O)cc2)[C@@]1(C)c1ccc(Cl)cc1</smiles>

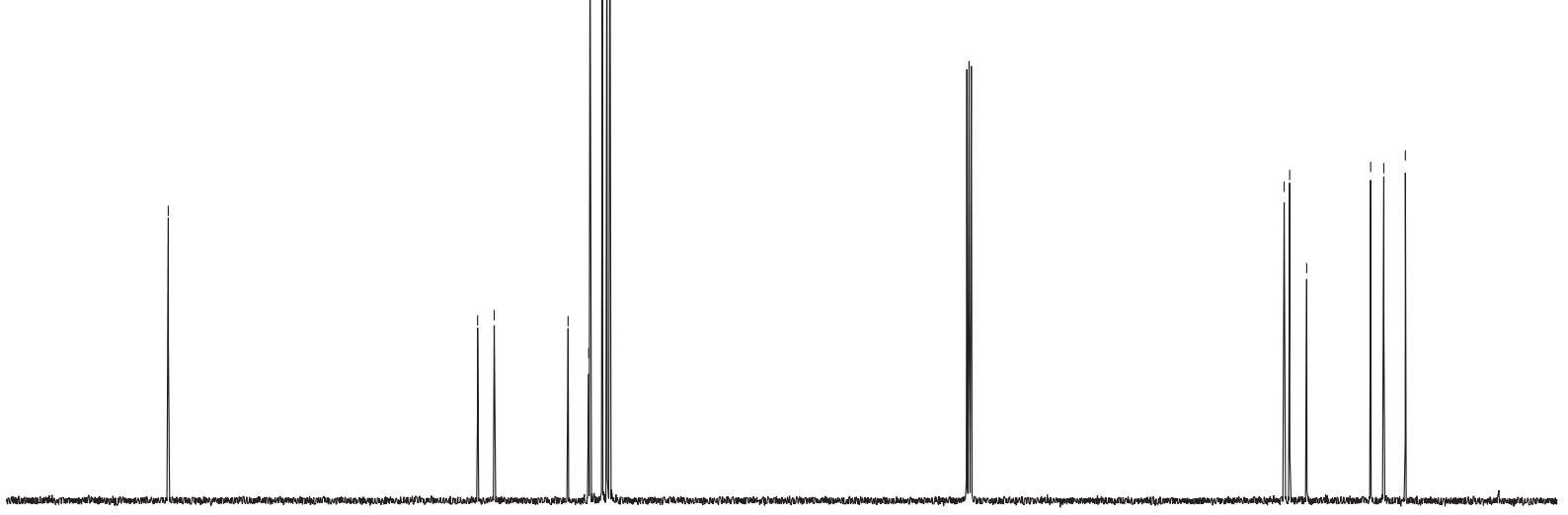

$\begin{array}{llllllllllllllllllllll}210 & 200 & 190 & 180 & 170 & 160 & 150 & 140 & 130 & 120 & \underset{\mathrm{f} 1(\mathrm{ppm})}{110} & 90 & 80 & 70 & 60 & 50 & 40 & 30 & 20 & 10 & 0\end{array}$ 

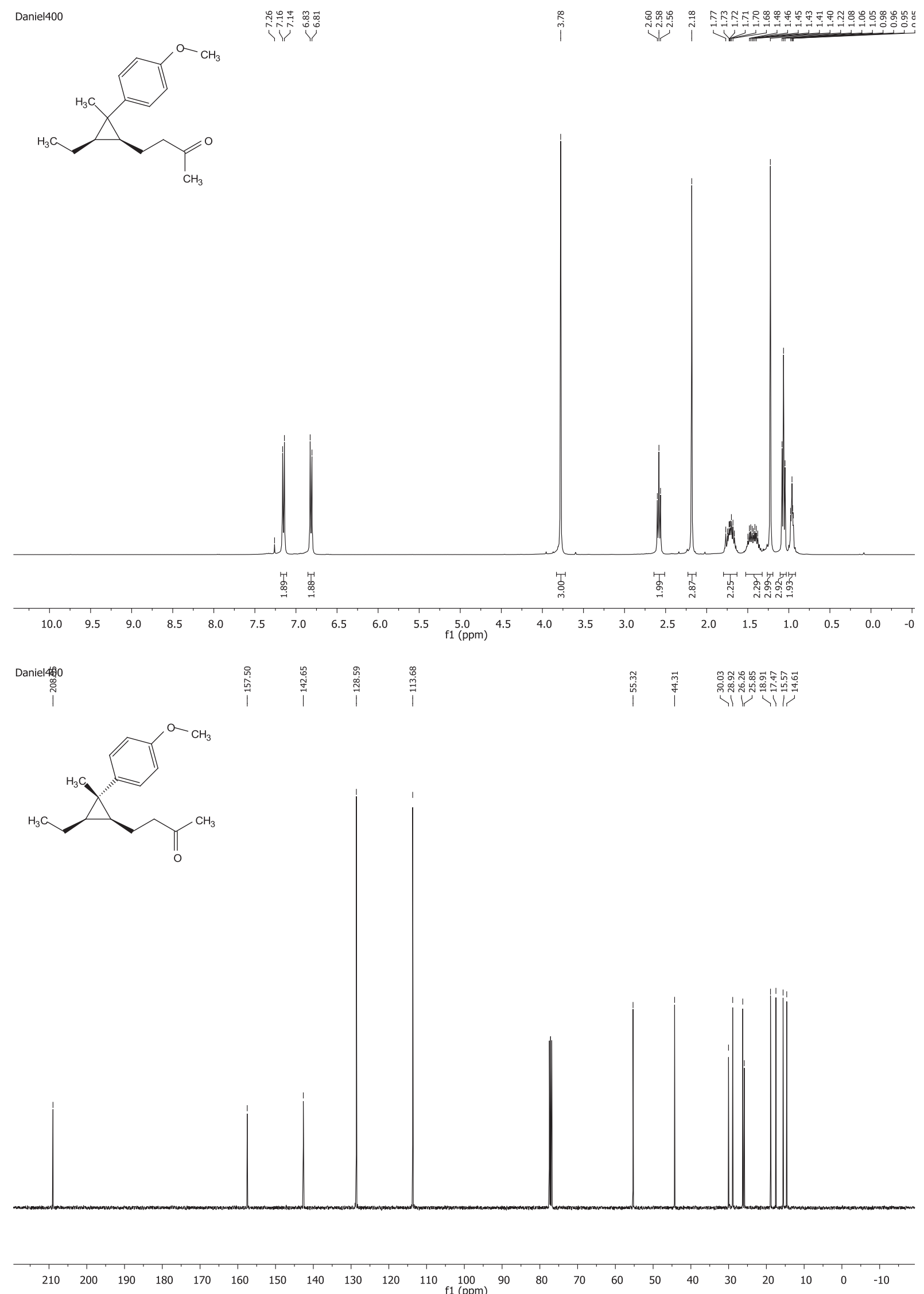

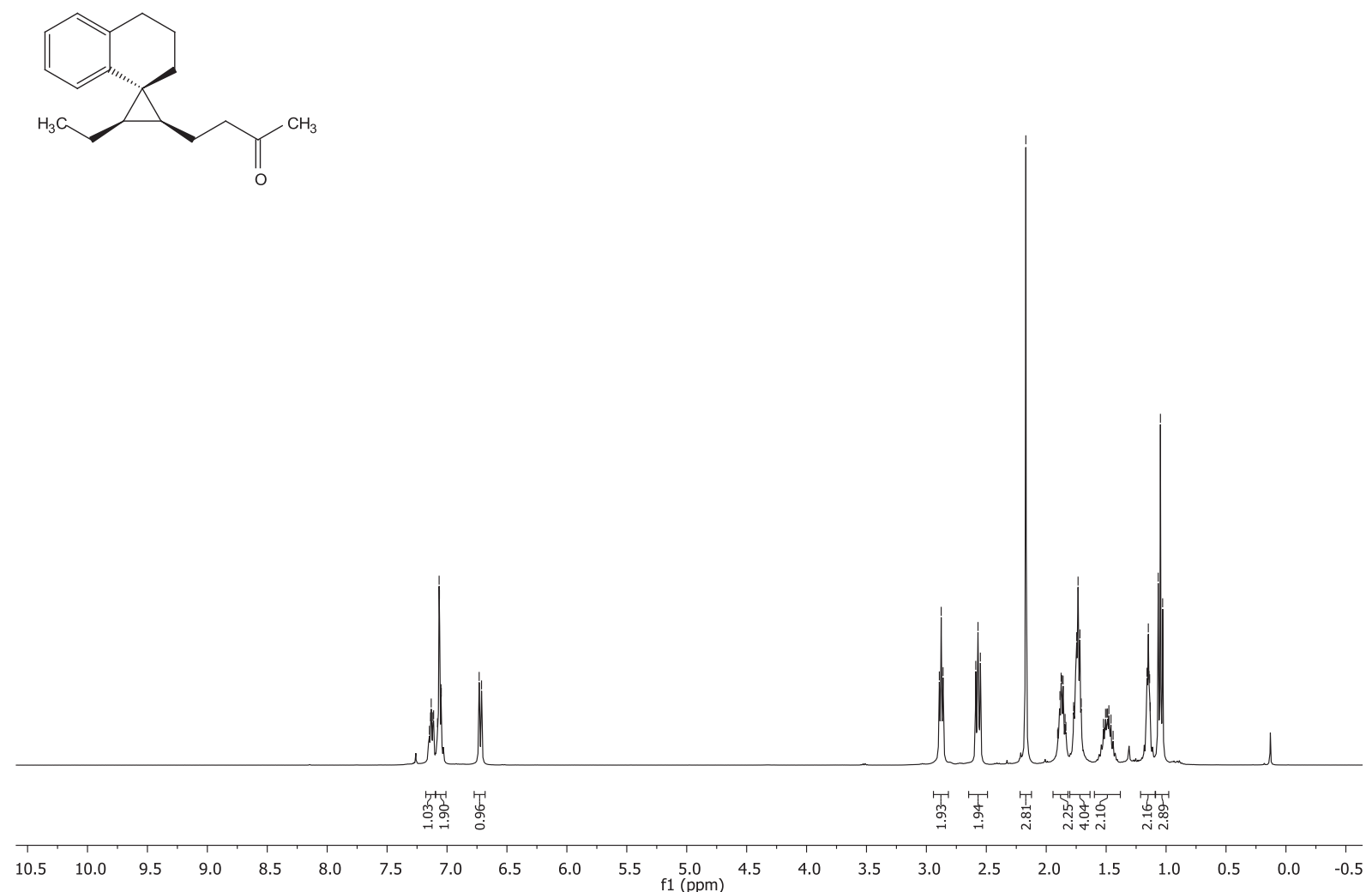

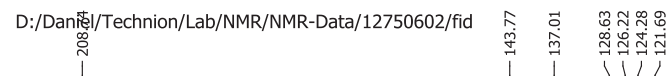

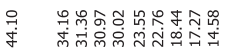

1111

।

(c)

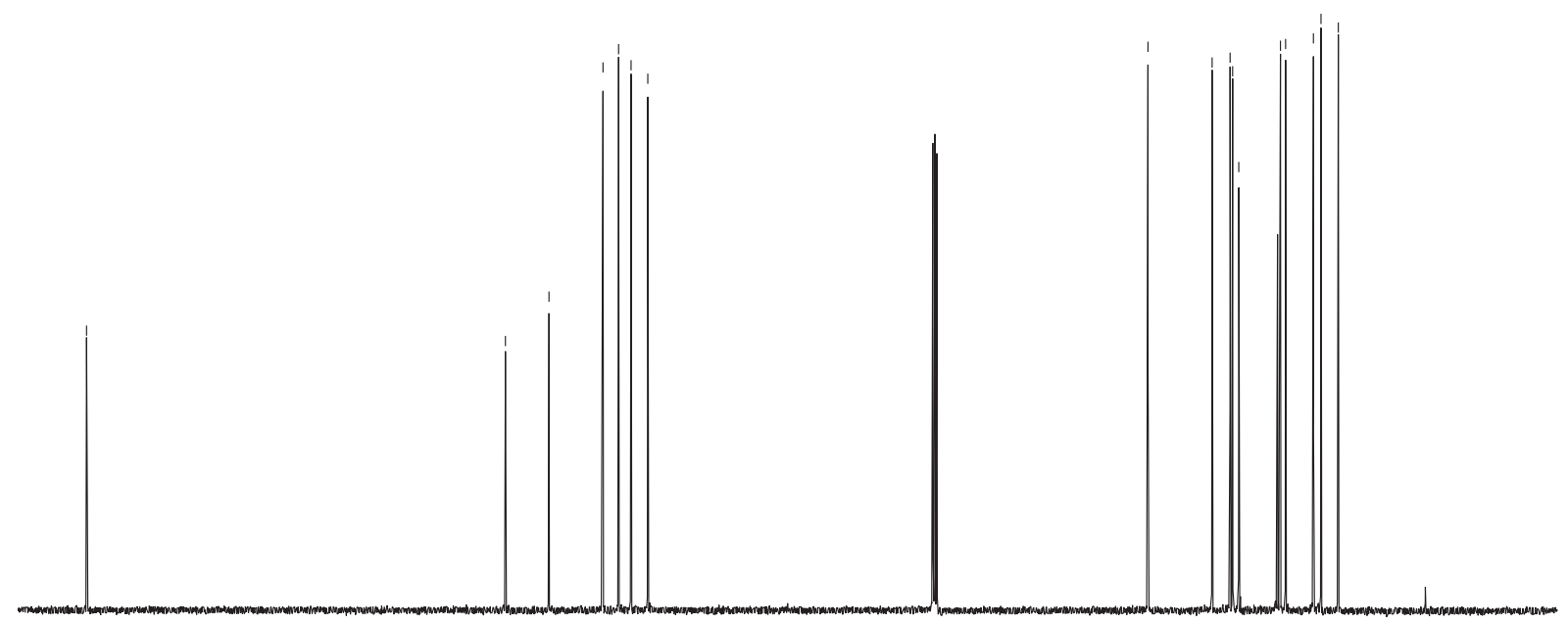

$\begin{array}{llllllllllllllllllllllllll}210 & 200 & 190 & 180 & 170 & 160 & 150 & 140 & 130 & 120 & 110 & 100 & 90 & 80 & 70 & 60 & 50 & 40 & 30 & 20 & 10 & 0 & -10\end{array}$ 

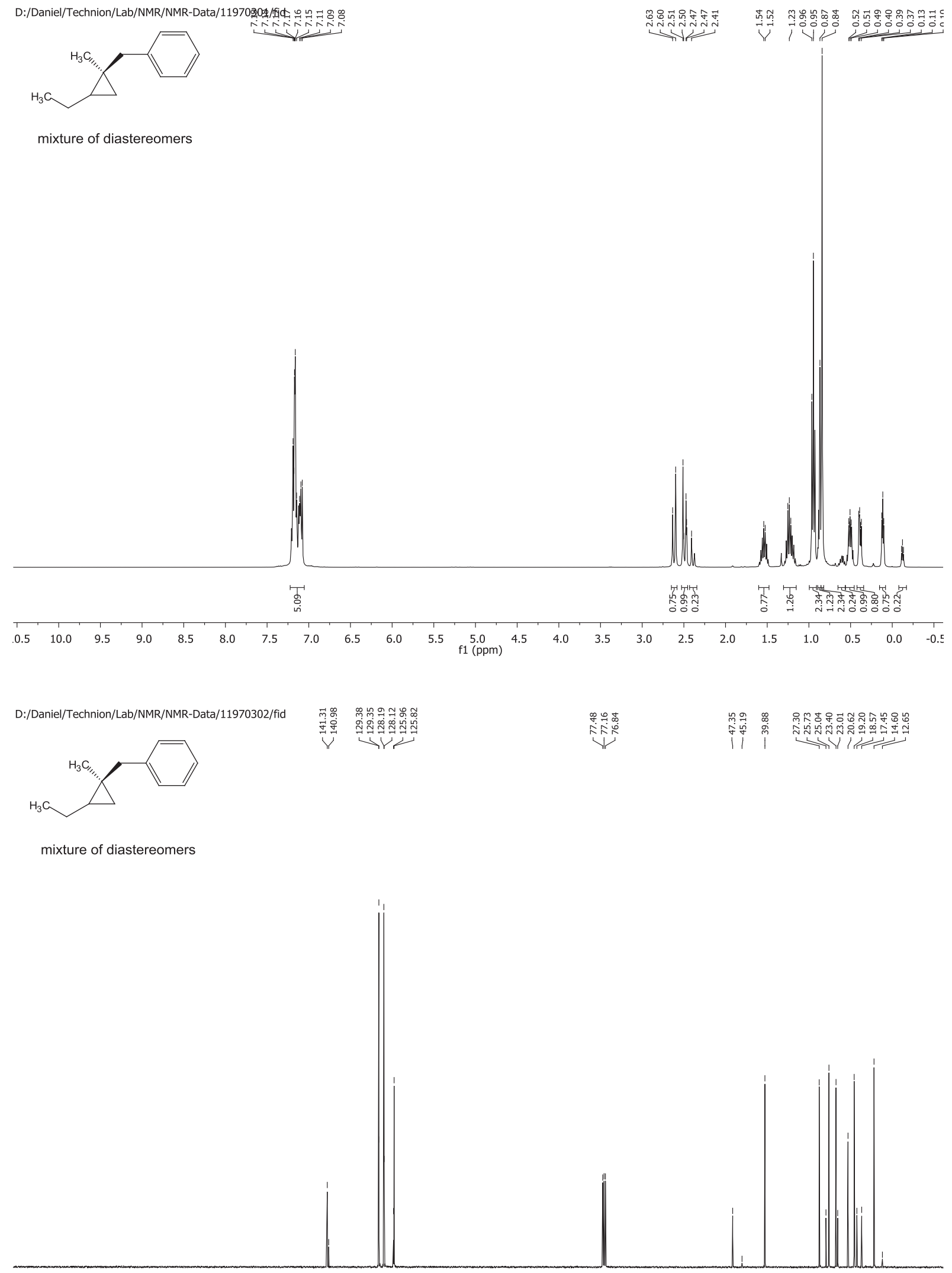

$\begin{array}{llllllllllllllllllllll}210 & 200 & 190 & 180 & 170 & 160 & 150 & 140 & 130 & 120 & 110 & 100 & 90 & 80 & 70 & 60 & 50 & 40 & 30 & 20 & 10 & 0\end{array}$ 

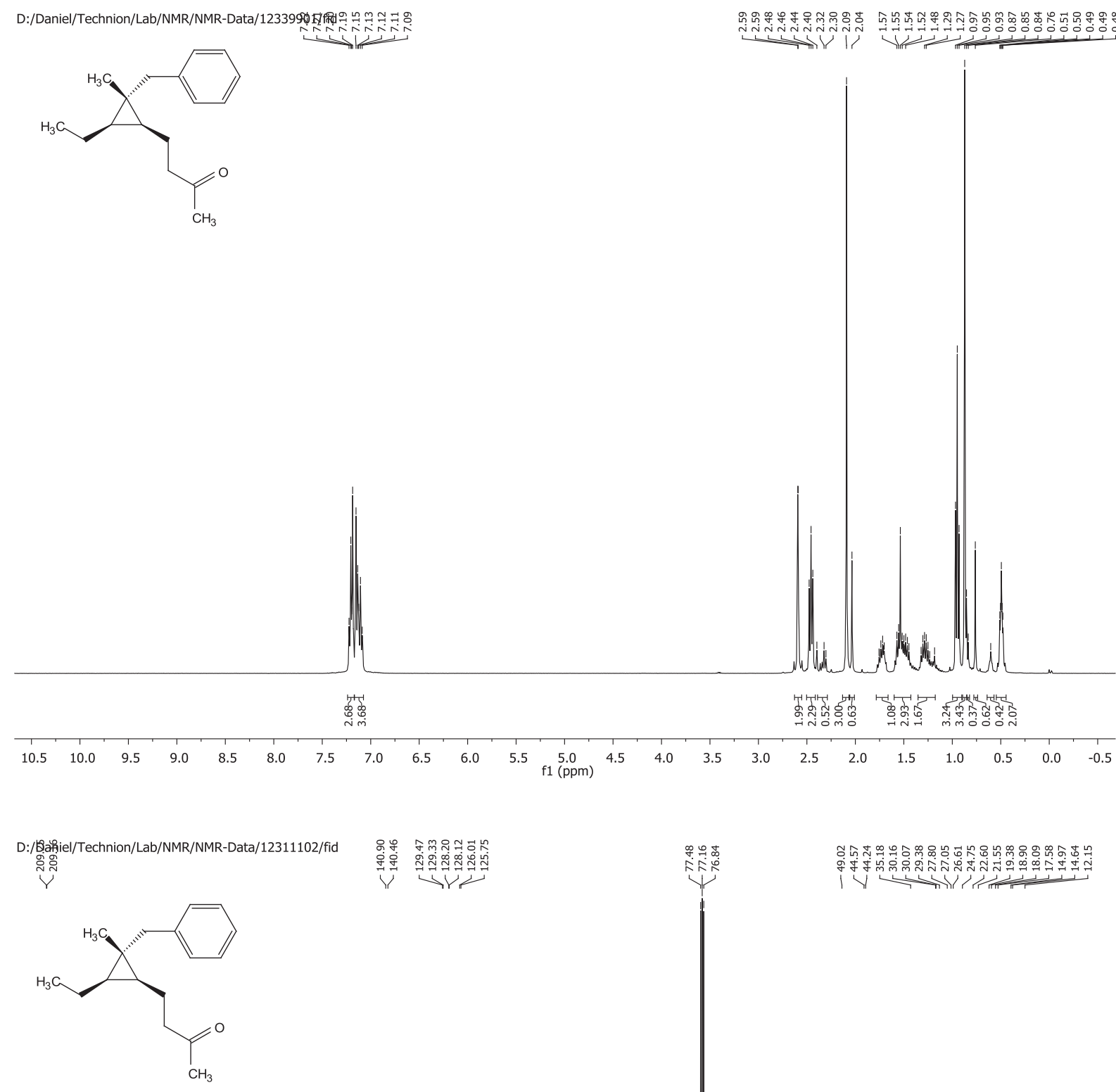

mixture of diastereomers

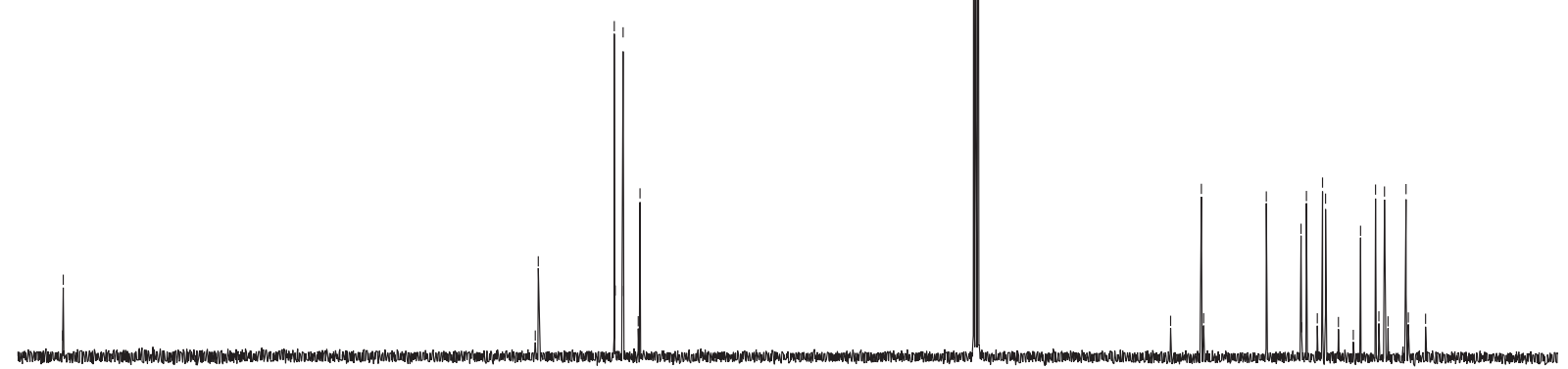

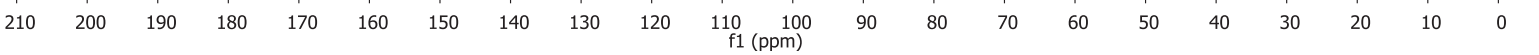



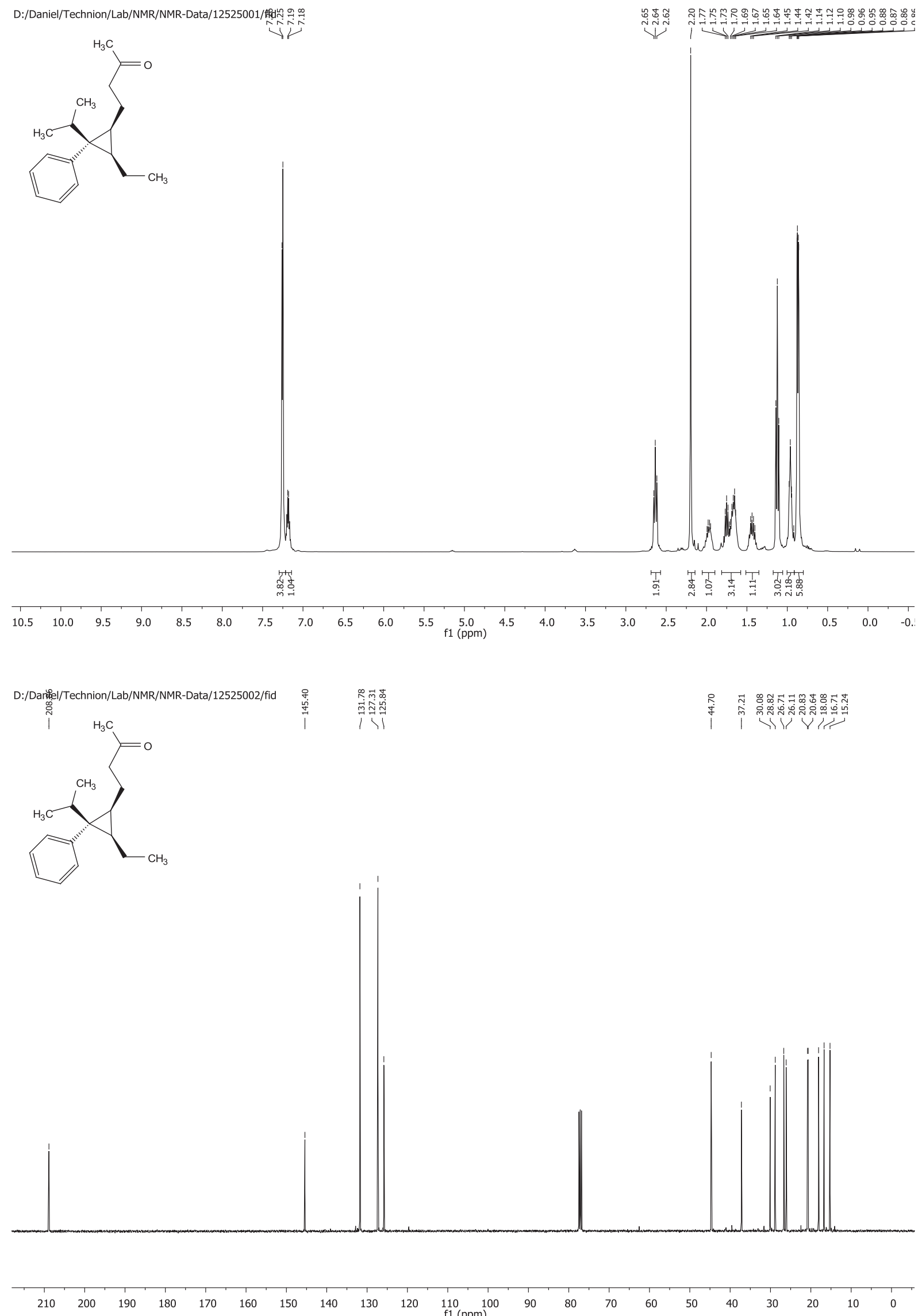
NOE's

$\mathrm{dd}, J=6.8,6.8 \mathrm{~Hz}$

$0.85 \quad, 7.16$

$q q, J=6.8,6.8 \mathrm{~Hz} \because{ }^{1}$

$2.62,2.18$

1.11
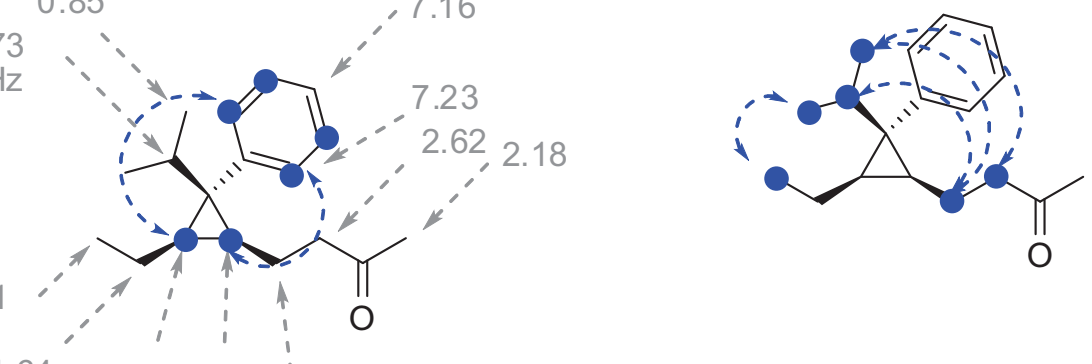

1.42 and 1.64

$0.95 \quad 0.95$

1.64 and 1.96

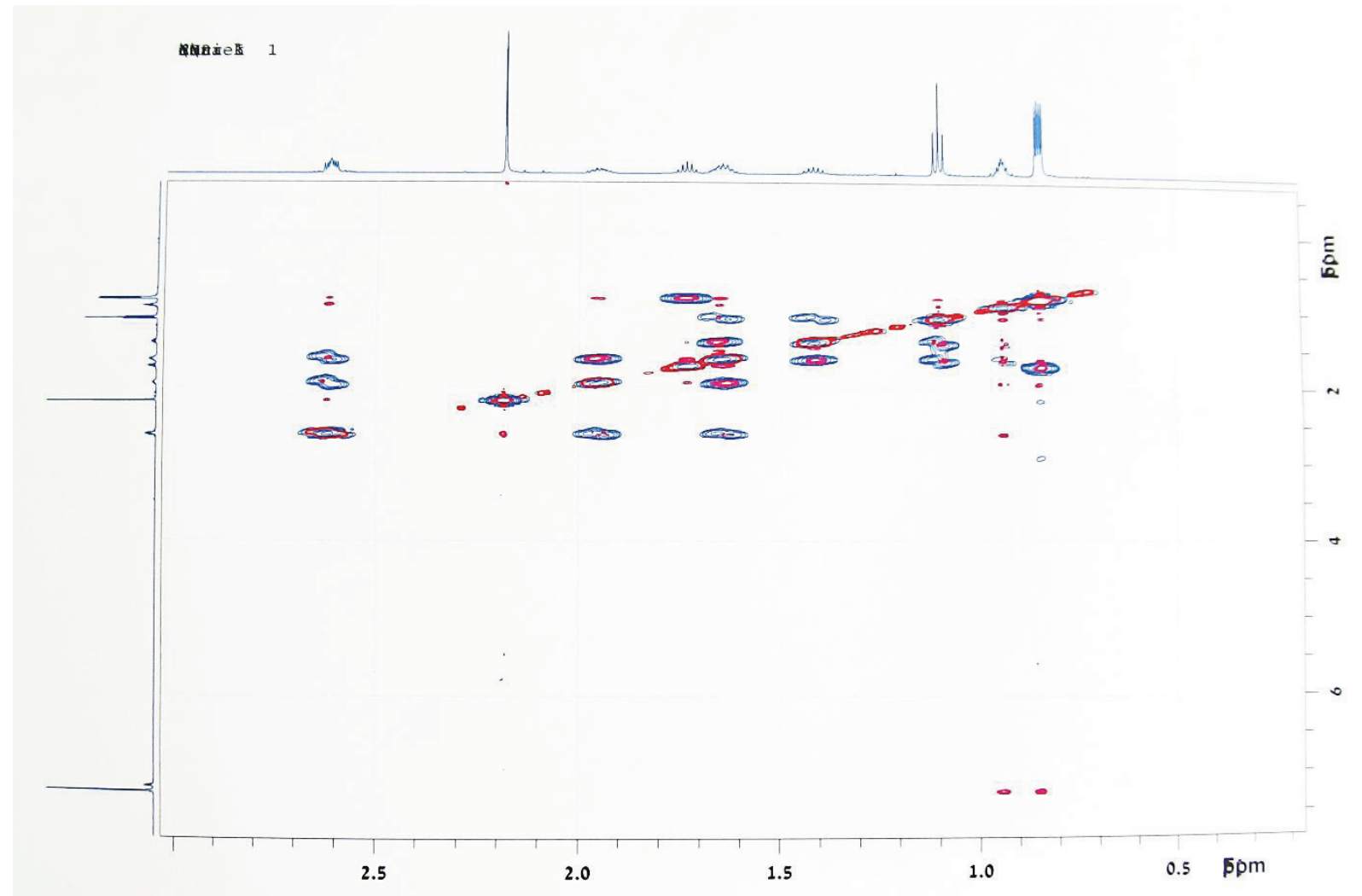

COSY Signals are shown in blue.

NOESY Signals are shown in red. 

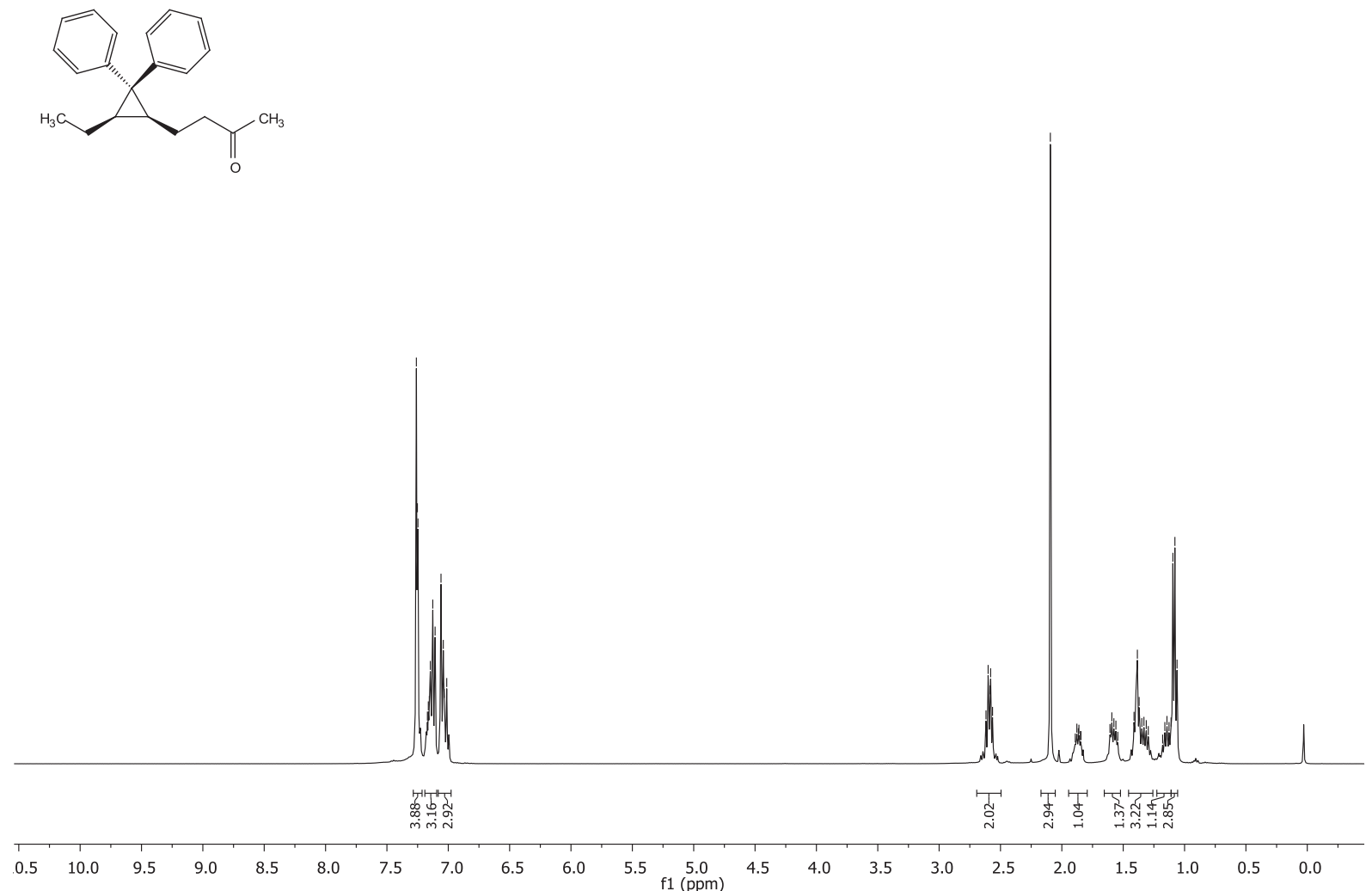

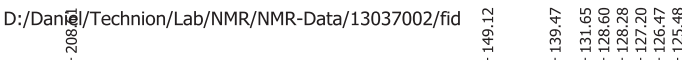
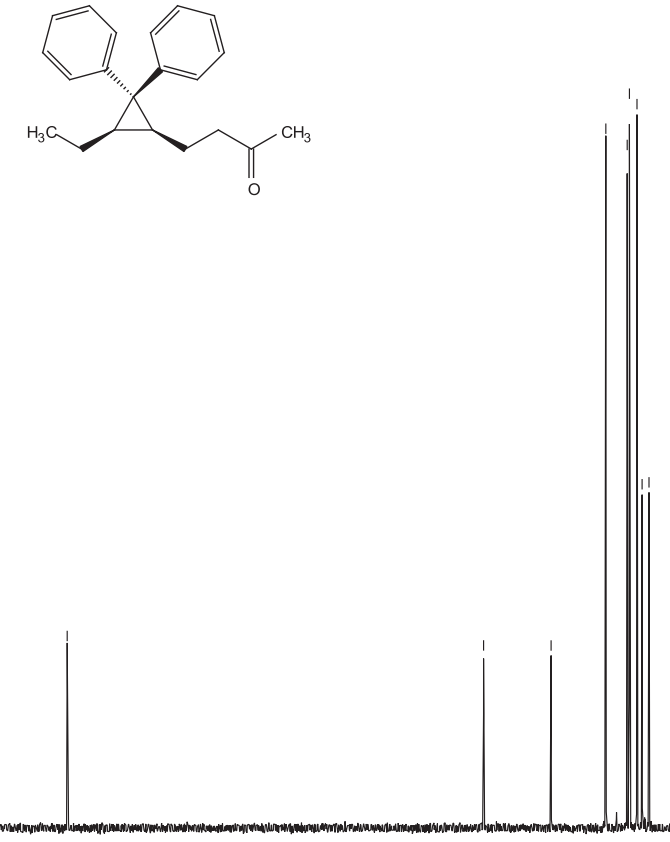

$\begin{array}{llllllllllll}210 & 200 & 190 & 180 & 170 & 160 & 150 & 140 & 130 & 120 & 110 & 100\end{array}$

$90 \quad 80$

70

6050

40

20

100 

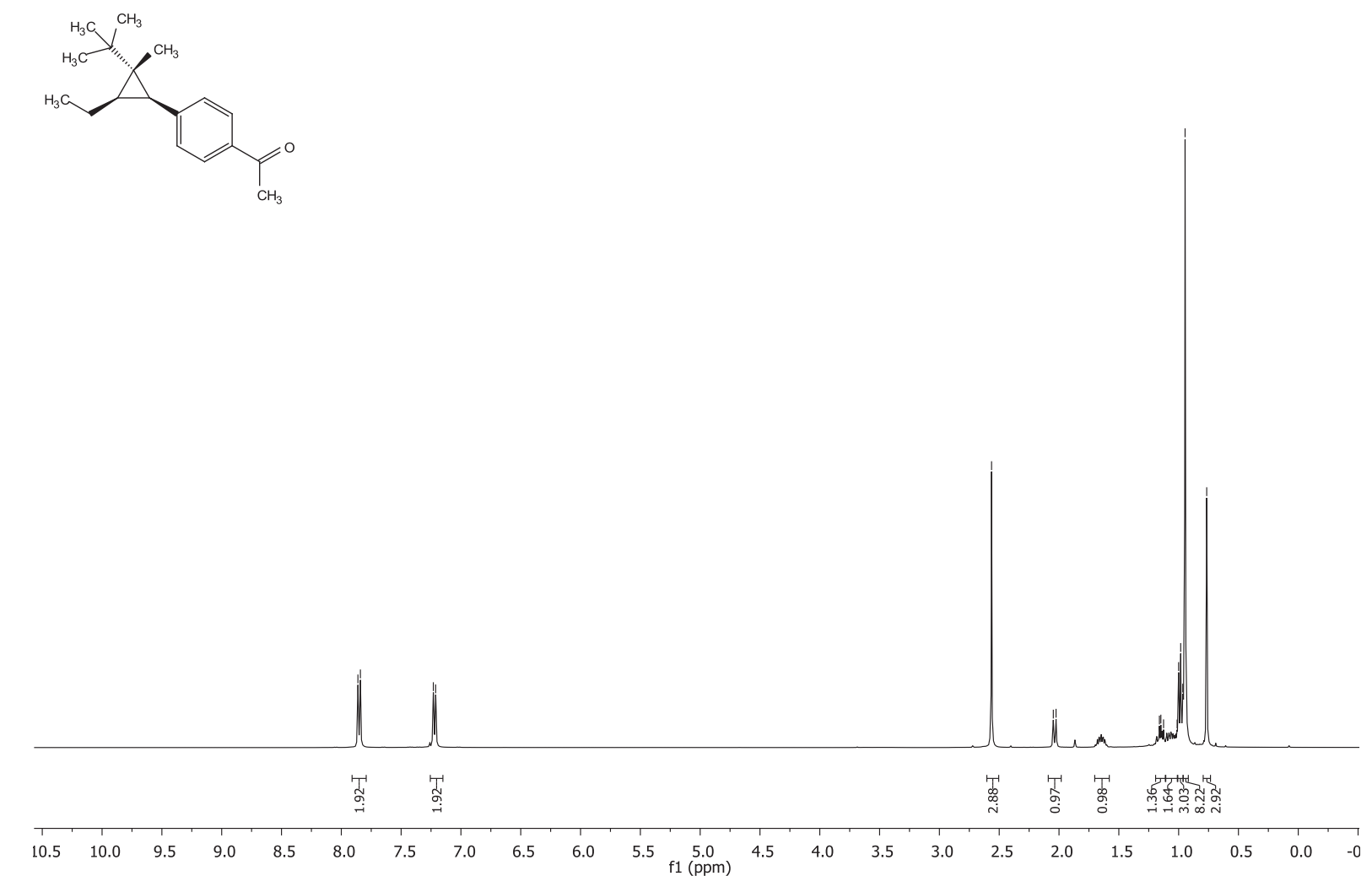

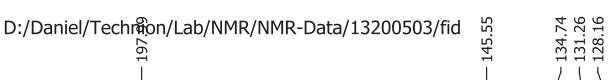

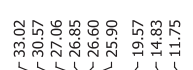
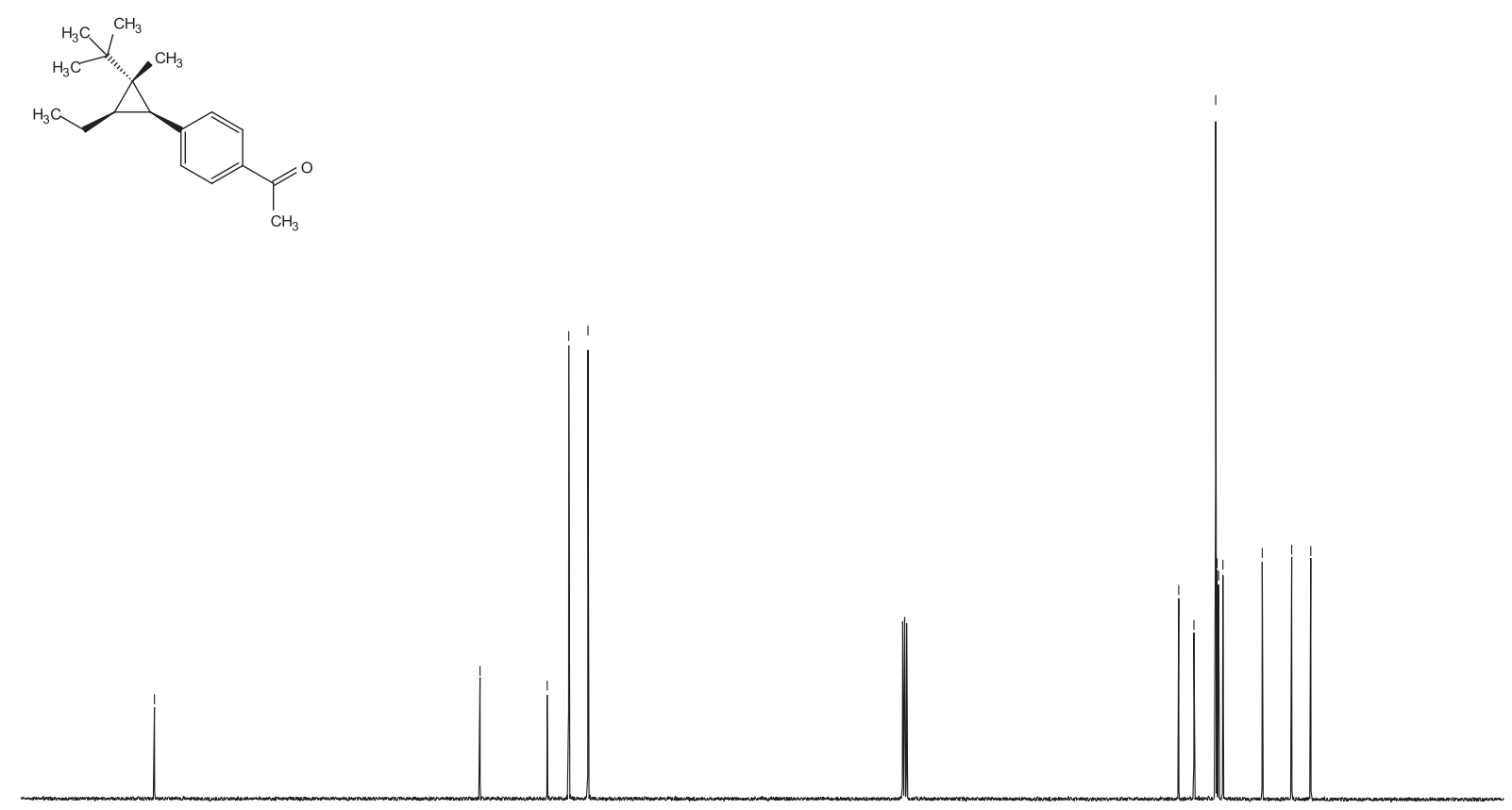

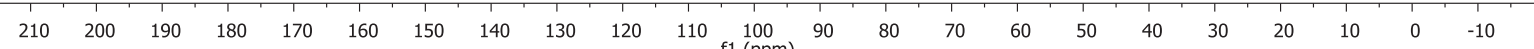

